Synthesis of perchlorinated sulfonium derivatives of closo-decaborate anion

$\left[2-\mathrm{B}_{10} \mathrm{Cl}_{9} \mathrm{SR}_{2}\right]^{-}\left(\mathrm{R}=i-\mathrm{C}_{3} \mathrm{H}_{7}, n-\mathrm{C}_{3} \mathrm{H}_{7}, n-\mathrm{C}_{4} \mathrm{H}_{9}, n-\mathrm{C}_{8} \mathrm{H}_{17}, n-\mathrm{C}_{12} \mathrm{H}_{25}, n-\mathrm{C}_{18} \mathrm{H}_{37}\right.$, $\mathrm{CH}_{2} \mathrm{Ph}$, cyclo-S $\left.\left(\mathrm{CH}_{2}\right)_{4}\right)$

Aleksei V. Golubev *, , Alexey S. Kubasov ${ }^{a}$, Alexander Yu. Bykov ${ }^{a}$, Konstantin Yu. Zhizhin ${ }^{a}$, Eleonora A. Kravchenko ${ }^{a}$, Andrei A. Gippius ${ }^{b, c}$, Sergey V. Zhurenko $^{c}$, Veronica A. Semenova ${ }^{d}$, Alexander A. Korlyukov ${ }^{e}$, Nikolay T.

$$
\begin{gathered}
\text { Kuznetsov }^{a} \\
\text { *golalekseival@mail.ru }
\end{gathered}
$$

${ }^{a}$ Kurnakov Institute of General and Inorganic Chemistry, Russian Academy of Sciences, Leninskii pr. 31, Moscow, 119991 Russia

${ }^{b}$ Lomonosov Moscow State University Faculty of Physics, Leninskiye Gory, Moscow, 119991 Russia

${ }^{c}$ P.N. Lebedev Physical Institute of the Russian Academy of Sciences, Leninskiy pr. 53, Moscow, 119991 Russia

${ }^{d}$ Moscow Institute of Physics and Technology National Research University, Institutskiy per. 9, Dolgoprudny, Moscow Region, 141701 Russia

${ }^{e}$ Nesmeyanova Institute of Organoelement Compounds, Russian Academy of Sciences, st. Vavilova 28, Moscow, 119991 Russia 


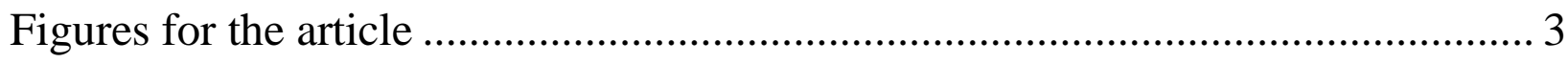

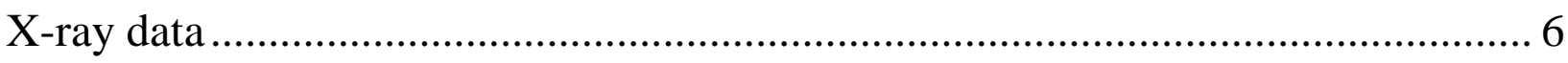

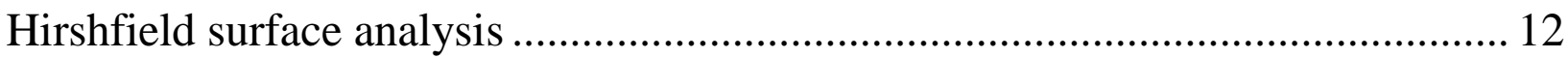

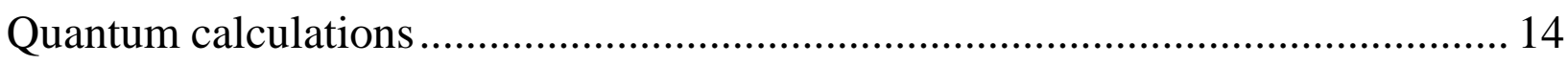

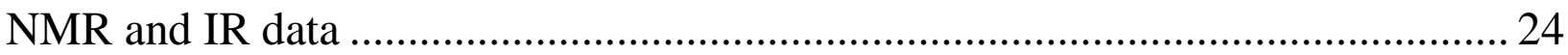

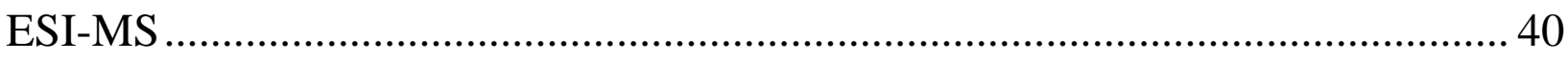

Figure depicting the discussed noncovalent $\mathrm{B}-\mathrm{Cl}$...H interactions ........................ 45 
Figures for the article

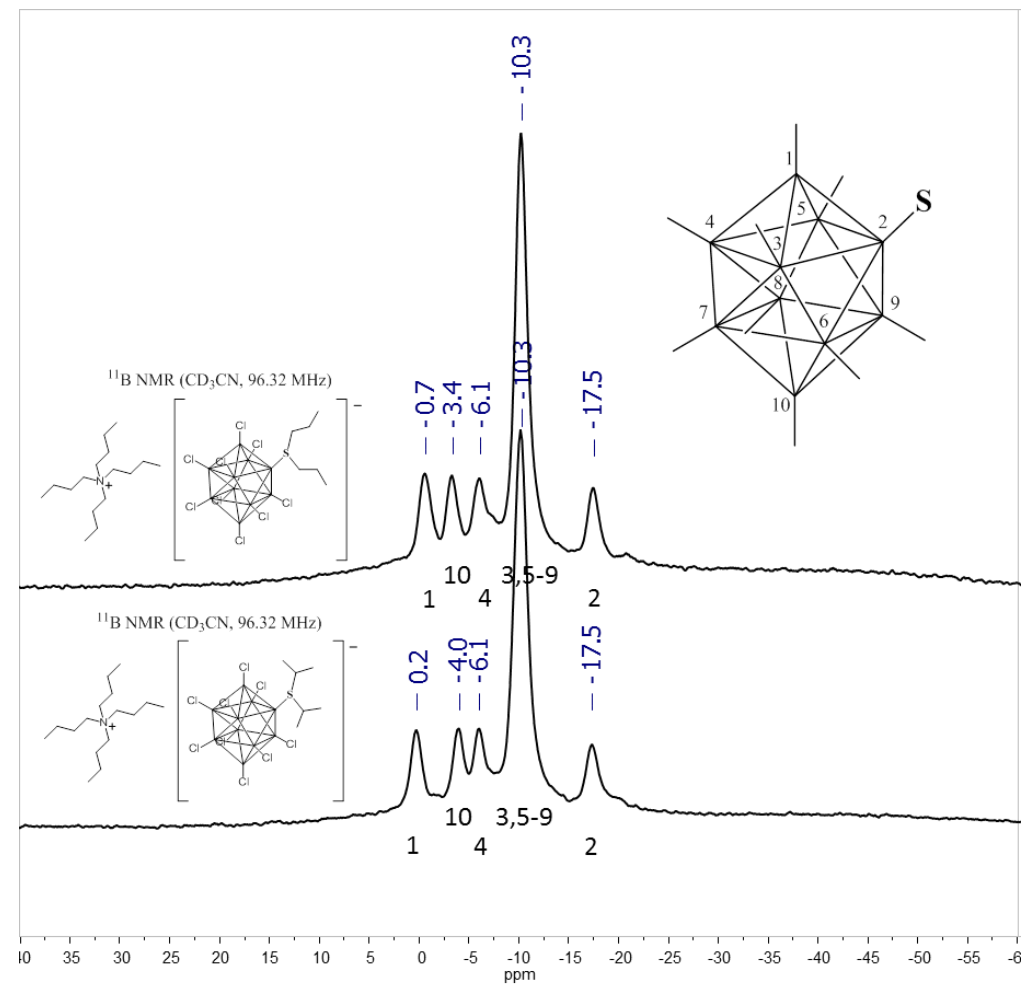

Fig. S1. ${ }^{11}$ B NMR spectrum (acetonitrile-d3, 96.32 MHz) of anions $\left[\mathrm{B}_{10} \mathrm{Cl} \mathrm{S}_{9}\left(n-\mathrm{C}_{3} \mathrm{H}_{7}\right)_{2}\right]^{-}$(top) and $\left[\mathrm{B}_{10} \mathrm{Cl}_{9} \mathrm{~S}\left(i-\mathrm{C}_{3} \mathrm{H}_{7}\right)_{2}\right]^{-}$(bottom)

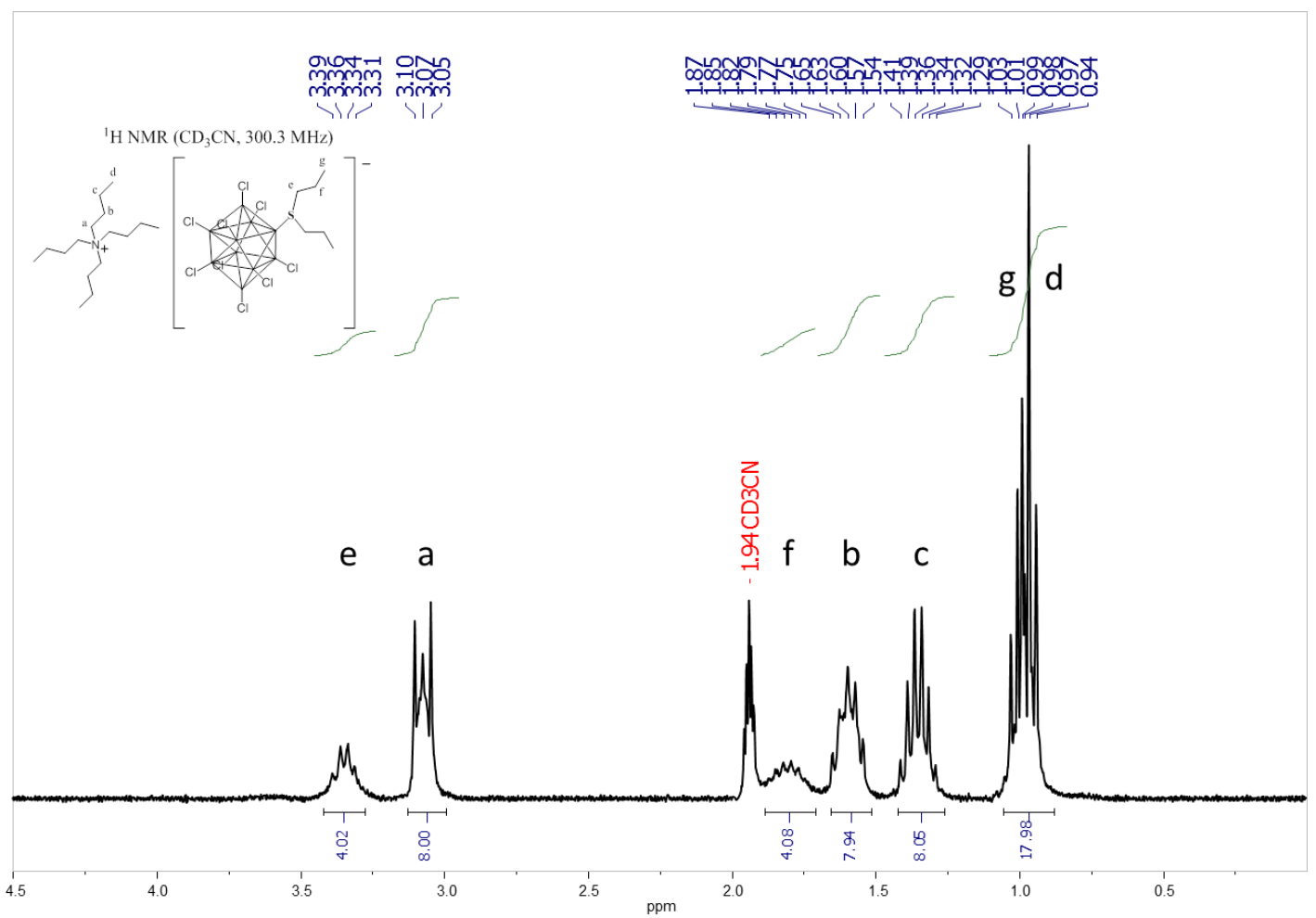

Fig. S2. Fragment ${ }^{1} \mathrm{H}$ NMR spectrum (acetonitrile-d3, 300.3 MHz) of $\left(\left(\mathrm{C}_{4} \mathrm{H}_{9}\right)_{4} \mathrm{~N}\right)\left[\mathrm{B}_{10} \mathrm{Cl}_{9} \mathrm{~S}\left(n-\mathrm{C}_{3} \mathrm{H}_{7}\right)_{2}\right]$ 


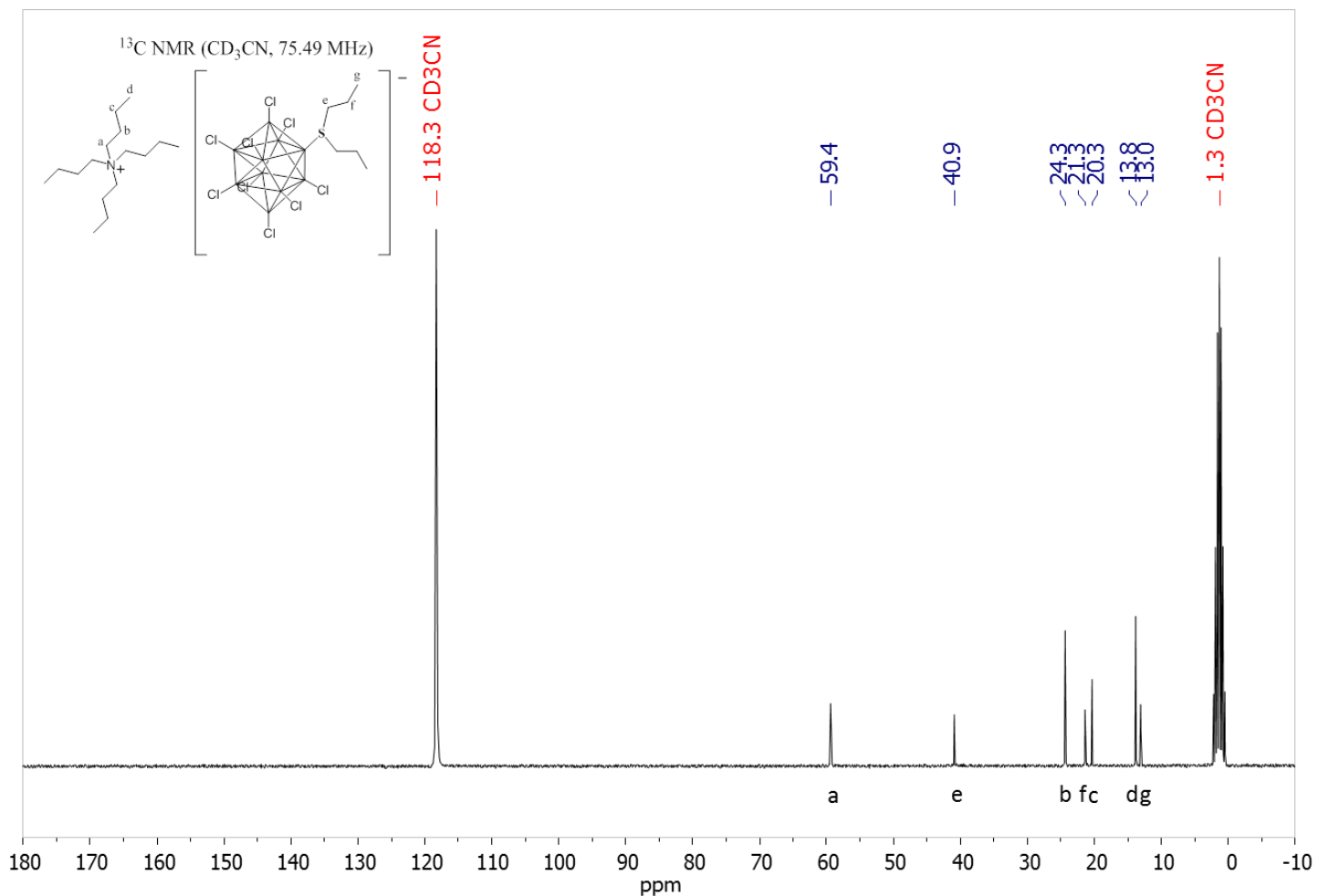

Fig. S3. ${ }^{13} \mathrm{C}$ NMR spectrum (acetonitrile-d3, 75.49 MHz) of $\left(\left(\mathrm{C}_{4} \mathrm{H}_{9}\right)_{4} \mathrm{~N}\right)\left[\mathrm{B}_{10} \mathrm{Cl}{ }_{9} \mathrm{~S}\left(n-\mathrm{C}_{3} \mathrm{H}_{7}\right)_{2}\right]$
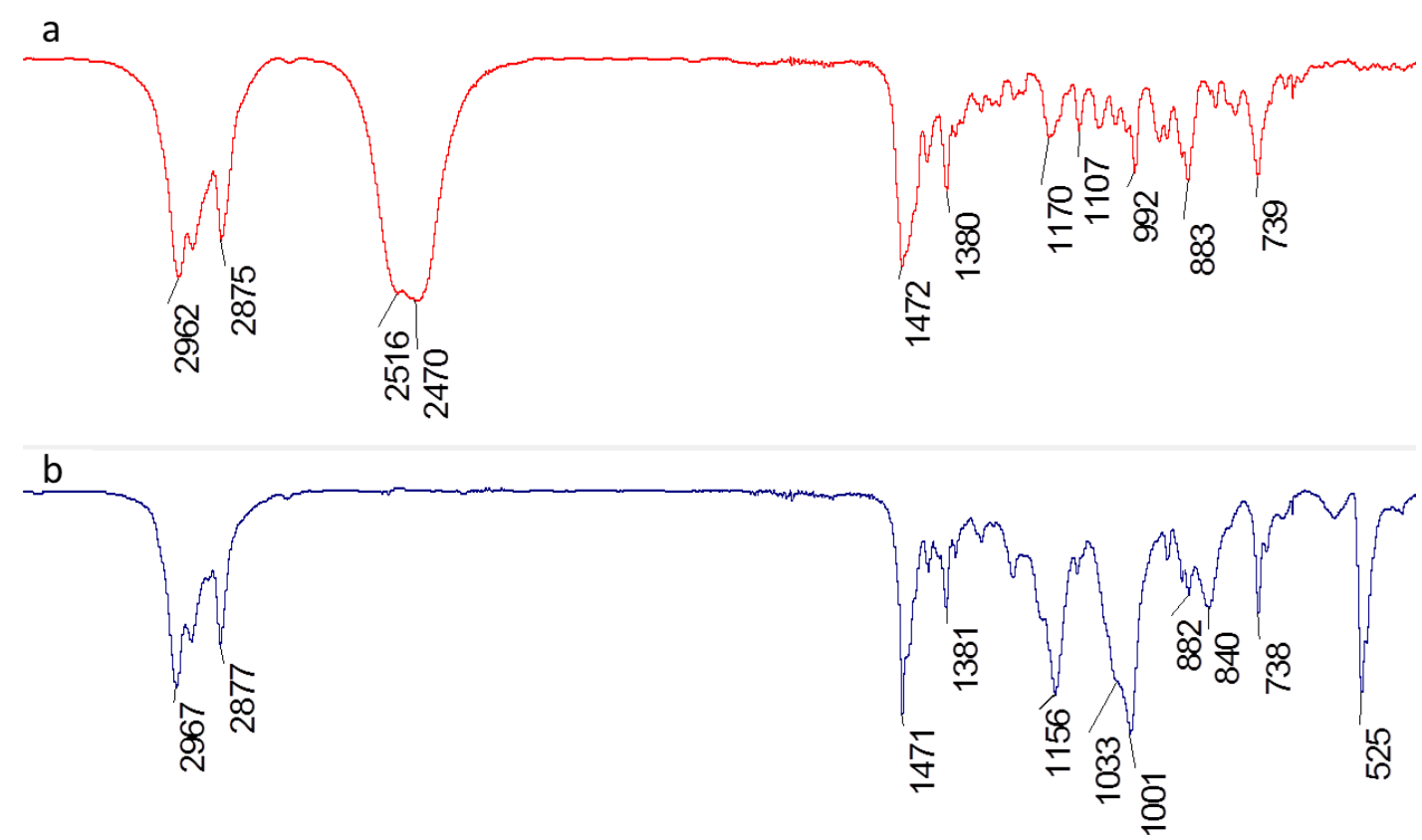

$\begin{array}{ccc}2500 & 2000 \\ \text { Wavenumbers (cm-1) } & 1500 & 1000 \\ & 500\end{array}$

Fig. S4. IR spectrum of $\left(\left(\mathrm{C}_{4} \mathrm{H}_{9}\right)_{4} \mathrm{~N}\right)\left[\mathrm{B}_{10} \mathrm{H}_{9} \mathrm{~S}\left(n-\mathrm{C}_{3} \mathrm{H}_{7}\right)_{2}\right]$ (a) and $\left(\left(\mathrm{C}_{4} \mathrm{H}_{9}\right)_{4} \mathrm{~N}\right)\left[\mathrm{B}_{10} \mathrm{Cl}_{9} \mathrm{~S}\left(n-\mathrm{C}_{3} \mathrm{H}_{7}\right)_{2}\right]$ (b) in tablets $\mathrm{KBr}$ 


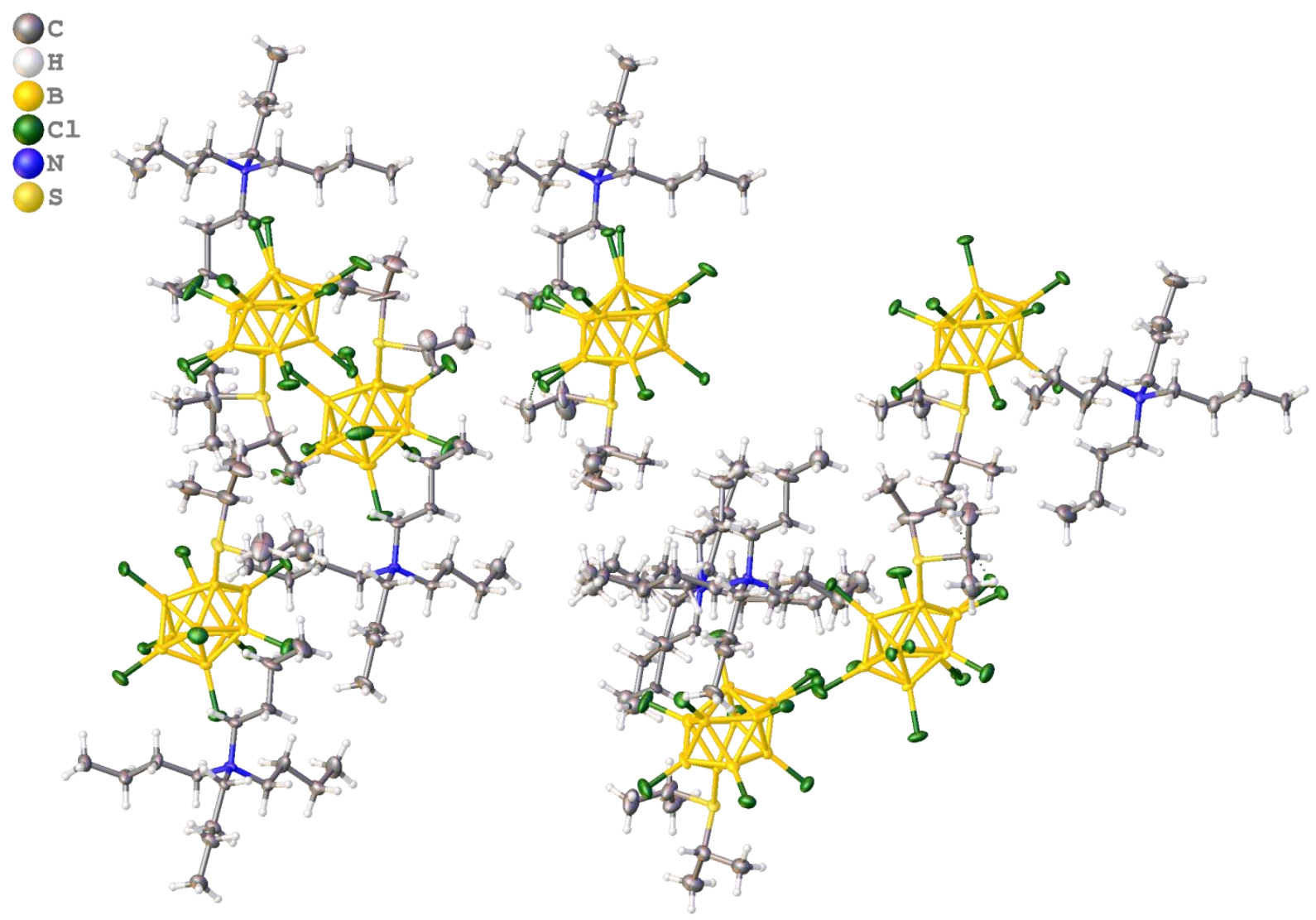

Fig. S5. Crystallographically independent part of the unit cell of compound 1. 


\section{X-ray data}

Table S1. Crystal data and structure refinement for compounds 1, 2, 7, and 8

\begin{tabular}{|c|c|c|}
\hline Structure & $\begin{array}{c}\left(\left(\mathrm{C}_{4} \mathrm{H}_{9}\right)_{4} \mathrm{~N}\right) \\
{\left[\mathrm{B}_{10} \mathrm{Cl}_{9} \mathrm{~S}\left(\mathrm{i}-\mathrm{C}_{3} \mathrm{H}_{7}\right)_{2}\right](\mathbf{1})}\end{array}$ & $\begin{array}{c}\left(\left(\mathrm{C}_{4} \mathrm{H}_{9}\right)_{4} \mathrm{~N}\right) \\
{\left[\mathrm{B}_{10} \mathrm{Cl}_{9} \mathrm{~S}\left(\mathrm{n}-\mathrm{C}_{3} \mathrm{H}_{7}\right)_{2}\right](2)}\end{array}$ \\
\hline Empirical formula & $\mathrm{C}_{22} \mathrm{H}_{50} \mathrm{~B}_{10} \mathrm{Cl}_{9} \mathrm{NS}$ & $\mathrm{C}_{22} \mathrm{H}_{50} \mathrm{~B}_{10} \mathrm{Cl}_{9} \mathrm{NS}$ \\
\hline Formula weight & 787.89 & 787.84 \\
\hline Temperature/K & 120 & 296 \\
\hline Crystal system & monoclinic & triclinic \\
\hline Space group & $\mathrm{C} 2 / \mathrm{c}$ & $\mathrm{P}-1$ \\
\hline $\mathrm{a} / \AA$ & $80.813(2)$ & $12.4414(4)$ \\
\hline $\mathrm{b} / \AA$ & $12.3592(4)$ & $12.4833(3)$ \\
\hline $\mathrm{c} / \AA$ & $55.5147(15)$ & $14.4736(4)$ \\
\hline$\alpha /^{\circ}$ & 90 & $110.7850(10)$ \\
\hline$\beta /{ }^{\circ}$ & $102.097(3)$ & $90.9070(10)$ \\
\hline$\gamma /{ }^{\circ}$ & 90 & $106.7690(10)$ \\
\hline Volume $/ \AA^{3}$ & $54216(3)$ & $1994.60(10)$ \\
\hline $\mathrm{Z}$ & 56 & 2 \\
\hline$\rho_{\text {calc }} \mathrm{g} / \mathrm{cm}^{3}$ & 1.351 & 1.312 \\
\hline$\mu / \mathrm{mm}^{-1}$ & 6.584 & 0.702 \\
\hline $\mathrm{F}(000)$ & 22849.0 & 816.0 \\
\hline Crystal size $/ \mathrm{mm}^{3}$ & $0.4 \times 0.4 \times 0.2$ & $0.7 \times 0.5 \times 0.2$ \\
\hline Radiation & $\operatorname{CuK} \alpha(\lambda=1.54178)$ & $\operatorname{MoK} \alpha(\lambda=0.71073)$ \\
\hline $2 \Theta$ range for data collection $/{ }^{\circ}$ & 2.236 to 144.458 & 4.176 to 59.994 \\
\hline Reflections collected & 305162 & 42030 \\
\hline Independent reflections & $51724\left[\mathrm{R}_{\mathrm{int}}=0.1301, \mathrm{R}_{\text {sigma }}=0.1012\right]$ & $\begin{array}{c}11480\left[\mathrm{R}_{\text {int }}=0.0272, \mathrm{R}_{\text {sigma }}\right. \\
=0.0290]\end{array}$ \\
\hline Data/restraints/parameters & $51724 / 28 / 2806$ & $11480 / 6 / 435$ \\
\hline Goodness-of-fit on $\mathrm{F}^{2}$ & 1.026 & 1.077 \\
\hline Final $\mathrm{R}$ indexes $[\mathrm{I}>=2 \sigma(\mathrm{I})]$ & $\mathrm{R}_{1}=0.0887, \mathrm{wR}_{2}=0.2014$ & $\mathrm{R}_{1}=0.0524, \mathrm{wR}_{2}=0.1124$ \\
\hline Final $\mathrm{R}$ indexes [all data] & $\mathrm{R}_{1}=0.1657, \mathrm{wR}_{2}=0.2537$ & $\mathrm{R}_{1}=0.0762, \mathrm{wR}_{2}=0.1281$ \\
\hline
\end{tabular}




\begin{tabular}{|c|c|c|}
\hline Structure & $\begin{array}{c}\left(\left(\mathrm{C}_{4} \mathrm{H}_{9}\right)_{4} \mathrm{~N}\right) \\
{\left[\mathrm{B}_{10} \mathrm{Cl}_{9} \mathrm{~S}\left(\mathrm{CH}_{2} \mathrm{Ph}\right)_{2}\right](7)}\end{array}$ & $\begin{array}{c}\left(\left(\mathrm{C}_{4} \mathrm{H}_{9}\right)_{4} \mathrm{~N}\right) \\
{\left[\mathrm{B}_{10} \mathrm{Cl}_{9}-c y c l o-\mathrm{S}\left(\mathrm{CH}_{2}\right)_{4}\right](\mathbf{8})}\end{array}$ \\
\hline Empirical formula & $\mathrm{C}_{34} \mathrm{H}_{56} \mathrm{~B}_{10} \mathrm{Cl}_{9} \mathrm{~N}_{3} \mathrm{~S}$ & $\mathrm{C}_{20} \mathrm{H}_{44} \mathrm{~B}_{10} \mathrm{Cl}_{9} \mathrm{NS}$ \\
\hline Formula weight & 966.02 & 757.77 \\
\hline Temperature/K & 296 & 296 \\
\hline Crystal system & triclinic & triclinic \\
\hline Space group & $\mathrm{P}-1$ & $\mathrm{P}-1$ \\
\hline 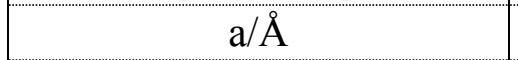 & $12.083(2)$ & $12.1472(9)$ \\
\hline $\mathrm{b} / \AA$ & $12.845(2)$ & $12.5139(9)$ \\
\hline $\mathrm{c} / \AA \AA$ & $16.576(3)$ & $15.1772(10)$ \\
\hline$\alpha /^{\circ}$ & $83.082(6)$ & $106.076(2)$ \\
\hline$\beta /{ }^{\circ}$ & $89.443(6)$ & $93.969(2)$ \\
\hline$\gamma /{ }^{\circ}$ & $79.991(6)$ & $118.982(3)$ \\
\hline Volume $/ \AA^{3}$ & $2515.0(8)$ & $1881.6(2)$ \\
\hline $\mathrm{Z}$ & 2 & 2 \\
\hline$\rho_{\text {calc }} \mathrm{g} / \mathrm{cm}^{3}$ & 1.276 & 1.337 \\
\hline$\mu / \mathrm{mm}^{-1}$ & 0.571 & 0.742 \\
\hline $\mathrm{F}(000)$ & 1000.0 & 780.0 \\
\hline Crystal size $/ \mathrm{mm}^{3}$ & $0.4 \times 0.4 \times 0.2$ & $0.6 \times 0.4 \times 0.2$ \\
\hline Radiation & $\operatorname{MoK} \alpha(\lambda=0.71073)$ & $\operatorname{MoK} \alpha(\lambda=0.71073)$ \\
\hline $2 \Theta$ range for data collection $/{ }^{\circ}$ & 4.202 to 51.998 & 3.998 to 60.986 \\
\hline Reflections collected & 18469 & 24595 \\
\hline Independent reflections & $\begin{array}{c}9684\left[\mathrm{R}_{\text {int }}=0.0382, \mathrm{R}_{\text {sigma }}=\right. \\
0.0762]\end{array}$ & $\begin{array}{c}11213\left[\mathrm{R}_{\text {int }}=0.0203, \mathrm{R}_{\text {sigma }}=\right. \\
0.0329]\end{array}$ \\
\hline Data/restraints/parameters & $9684 / 0 / 520$ & $11213 / 0 / 381$ \\
\hline Goodness-of-fit on $\mathrm{F}^{2}$ & 0.989 & 1.016 \\
\hline Final $\mathrm{R}$ indexes $[\mathrm{I}>=2 \sigma(\mathrm{I})]$ & $\mathrm{R}_{1}=0.0522, \mathrm{wR}_{2}=0.1299$ & $\mathrm{R}_{1}=0.0446, w \mathrm{R}_{2}=0.1050$ \\
\hline Final $\mathrm{R}$ indexes [all data] & $\mathrm{R}_{1}=0.1153, \mathrm{wR}_{2}=0.1548$ & $\mathrm{R}_{1}=0.0653, \mathrm{wR}_{2}=0.1155$ \\
\hline
\end{tabular}




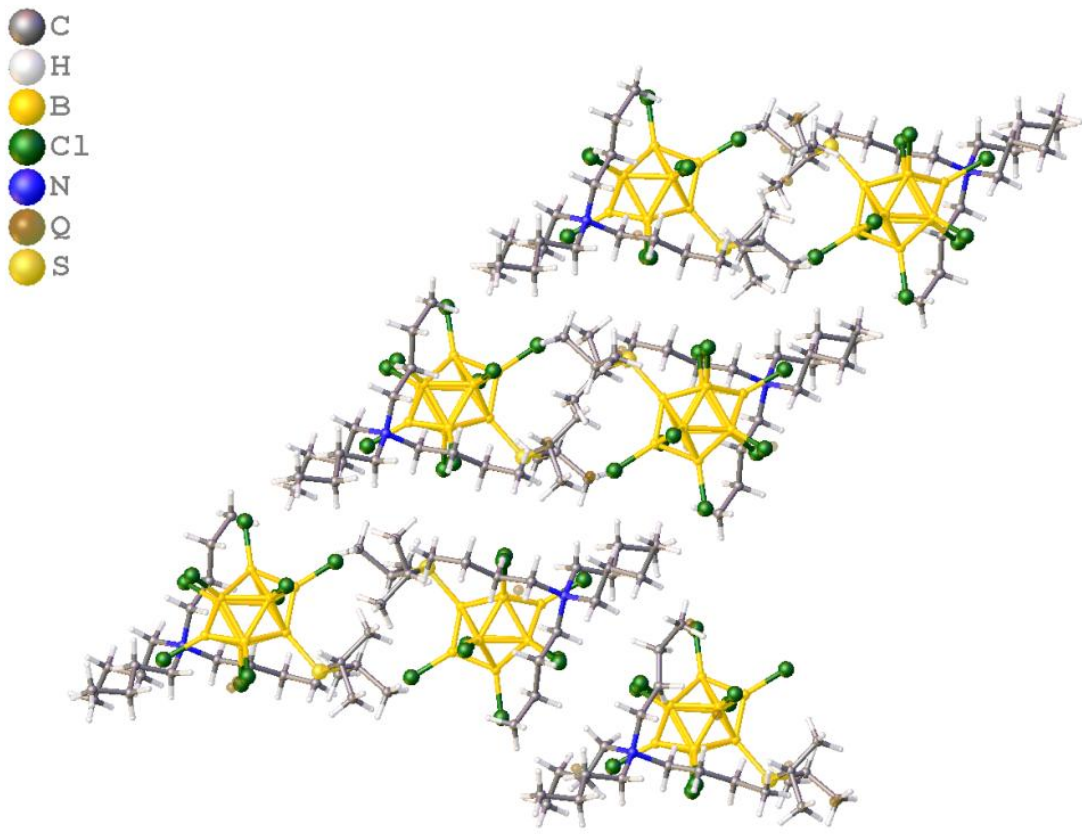

Fig. S6. Packing of compound 1 parallel to plane ac
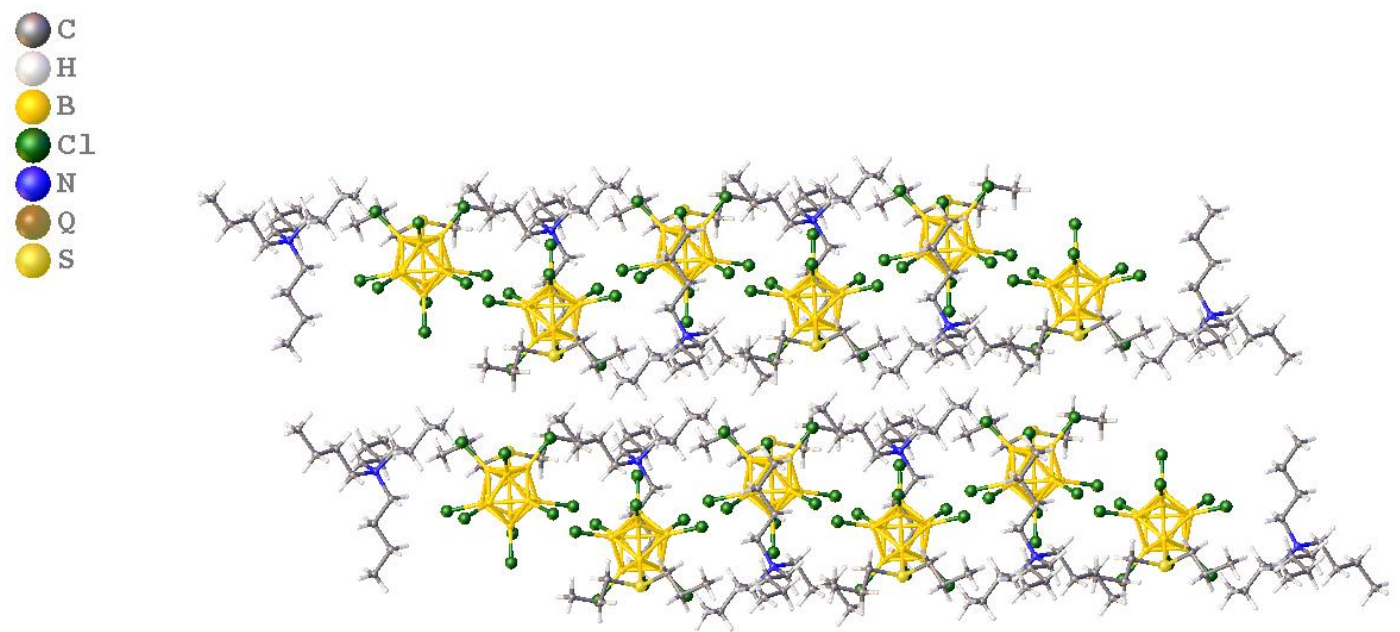

Fig. S7. Packing of compound 2 parallel to plane ab 

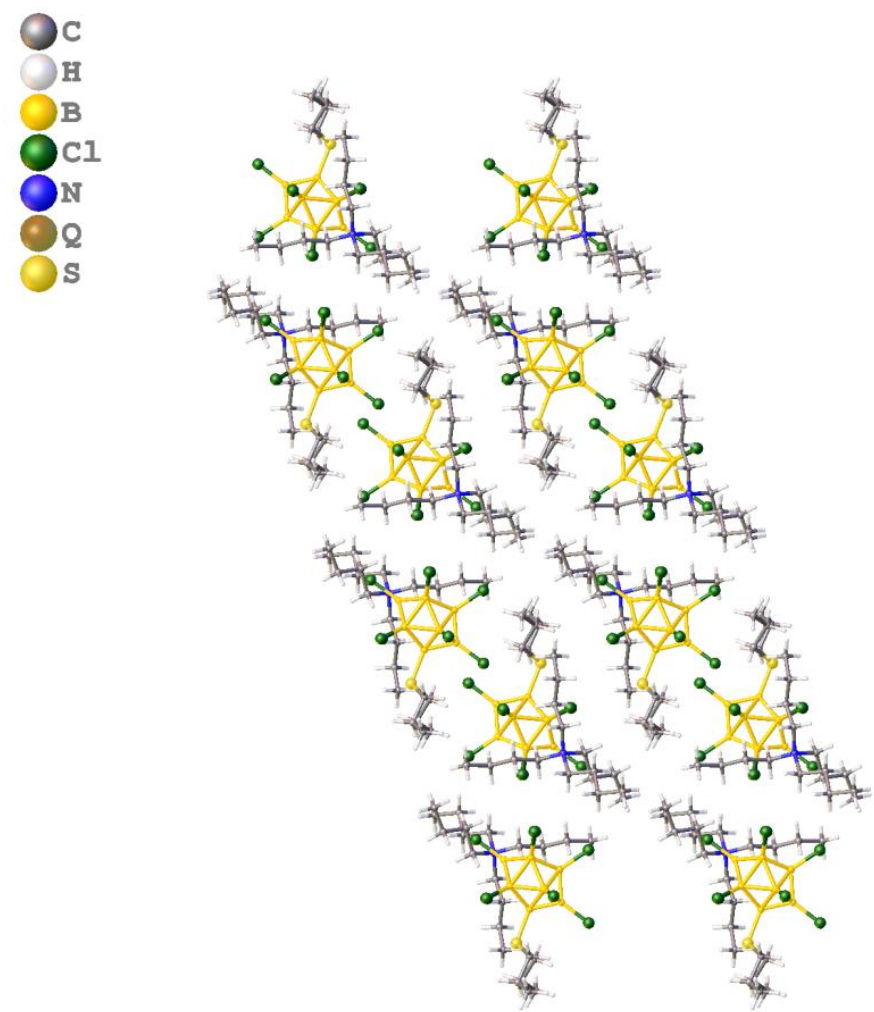

Fig. S8. Packing of compound 2 parallel to plane bc
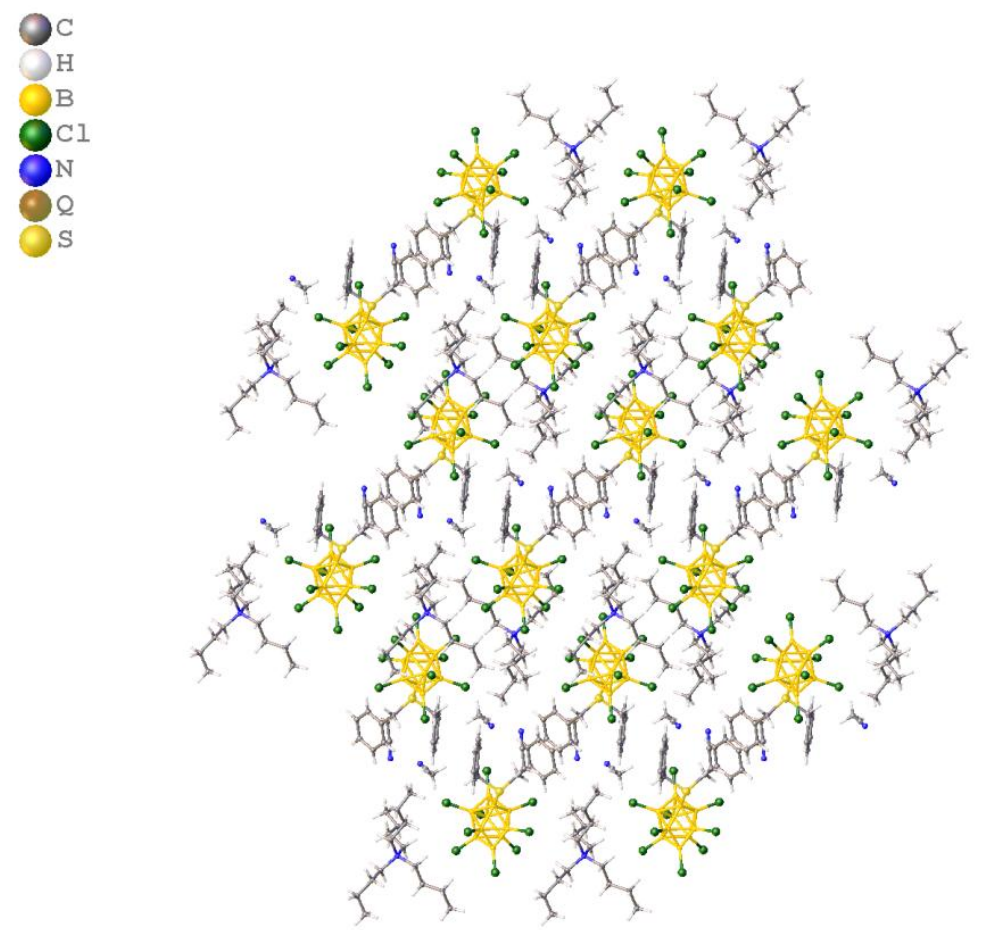

Fig. S9. Packing of compound 7 parallel to plane bc 

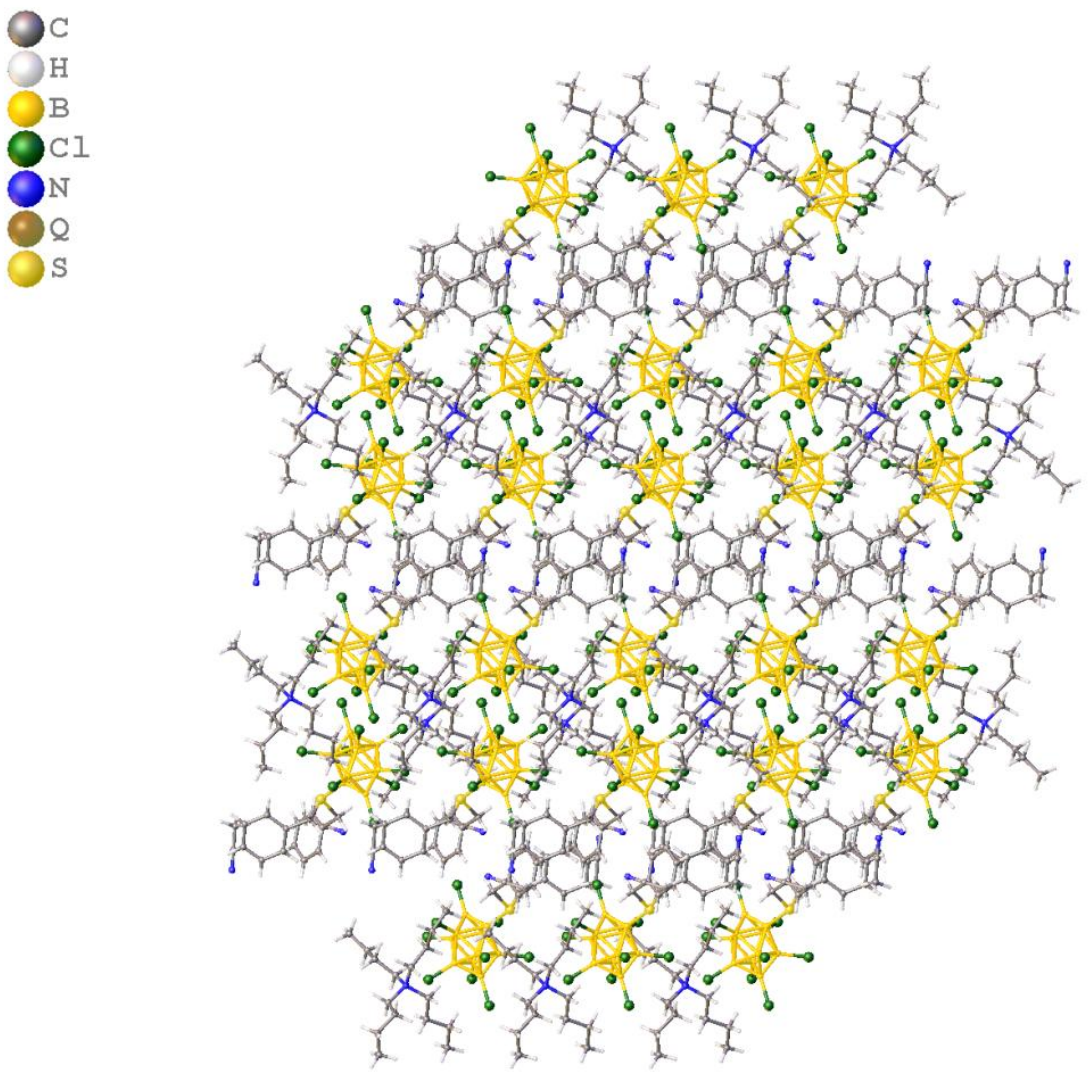

Fig. S10. Packing of compound $\mathbf{7}$ parallel to plane $\mathbf{1 1 0}$

$8^{\circ}$

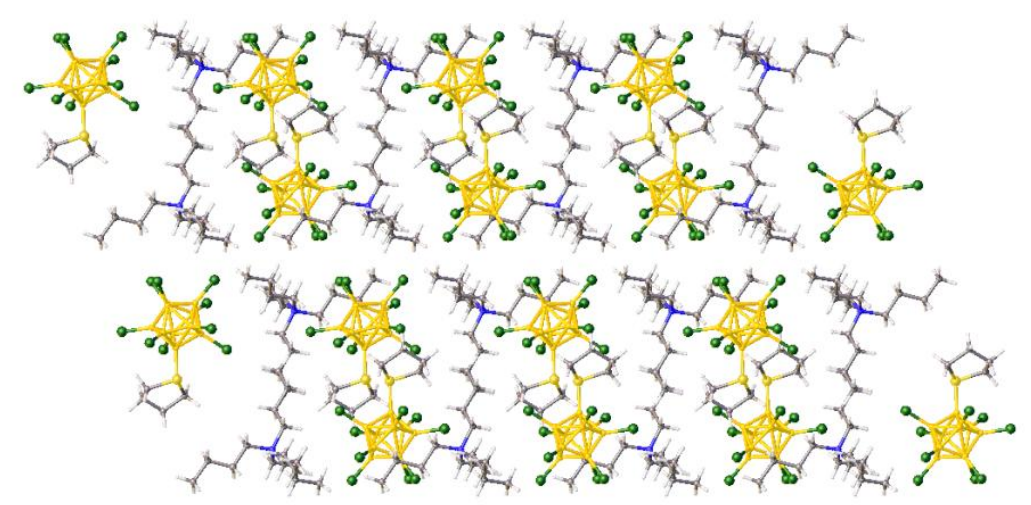

Fig. S11. Packing of compound 8 parallel to plane bc 


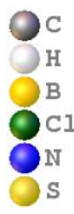

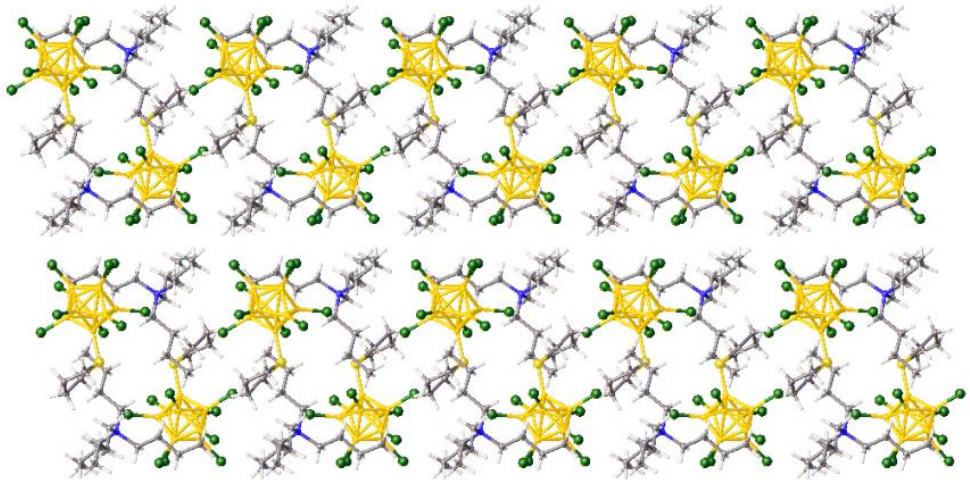

Fig. S12. Packing of compound $\mathbf{8}$ parallel to plane $\mathbf{1 1 0}$ 


\section{Hirshfield surface analysis}

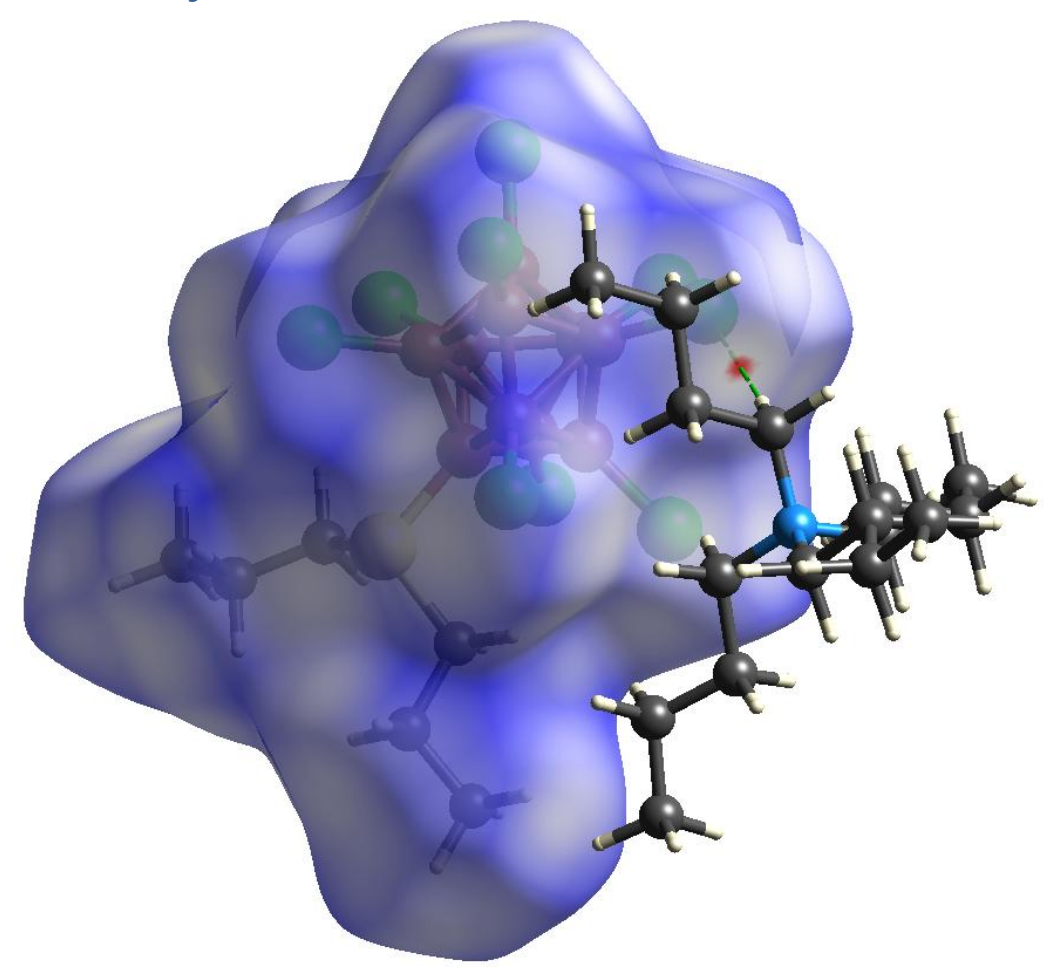

Fig. S13. Visualization of Hirshfeld surfaces for X-ray diffraction structure of compound $\mathbf{2}$ for different orientation of $n$-propyl fragments.
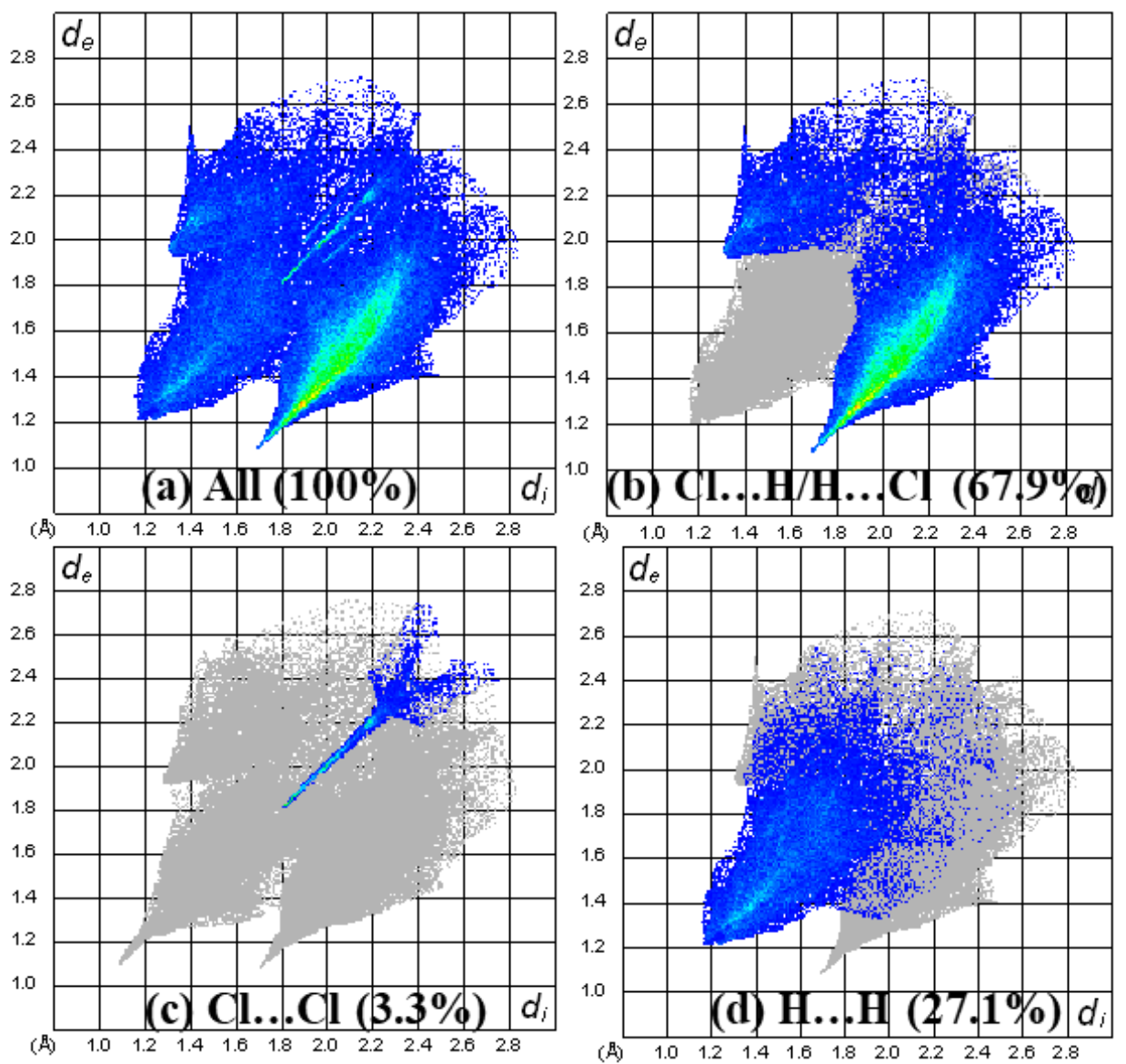

Fig. S14. (a) Full two-dimensional fingerprint plot for compound 2, together with that delineated into (b) $\mathrm{Cl}$... H/H...Cl, (c) $\mathrm{Cl}$...Cl, (d) $\mathrm{H} \ldots . . \mathrm{H}$ contacts for different orientation of of $n$-propyl fragments. 


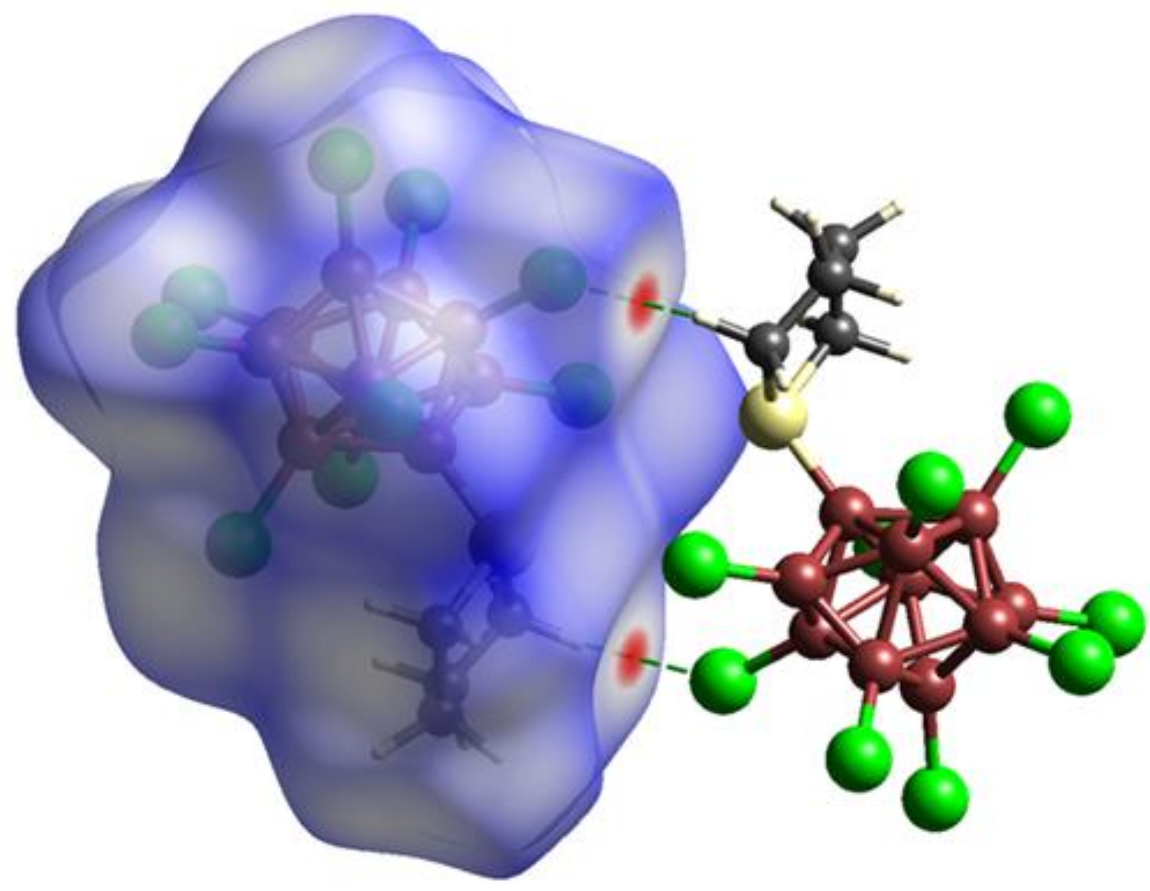

Fig. S15. Visualization of Hirshfeld surfaces for X-ray diffraction structure of compound $\mathbf{8}$ for different orientation of tetrahydrothiophene fragment.
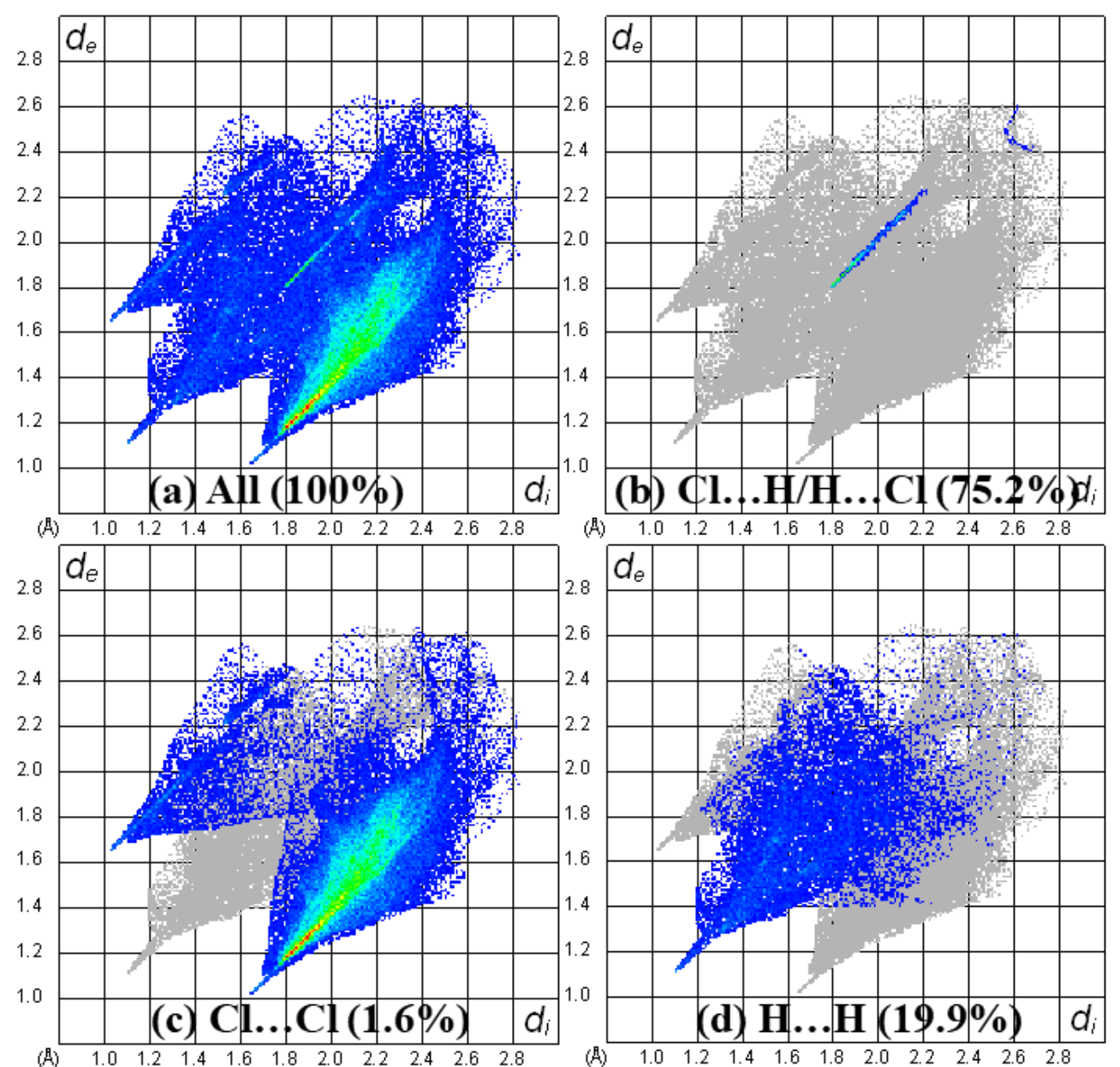

Fig. S16. (a) Full two-dimensional fingerprint plot for compound 8, together with that delineated into (b) $\mathrm{Cl} \ldots \mathrm{H} / \mathrm{H} \ldots \mathrm{Cl},(\mathrm{c}) \mathrm{Cl} . . \mathrm{Cl}$, (d) $\mathrm{H} . . . \mathrm{H}$ contacts for different orientation of tetrahydrothiophene fragment. 


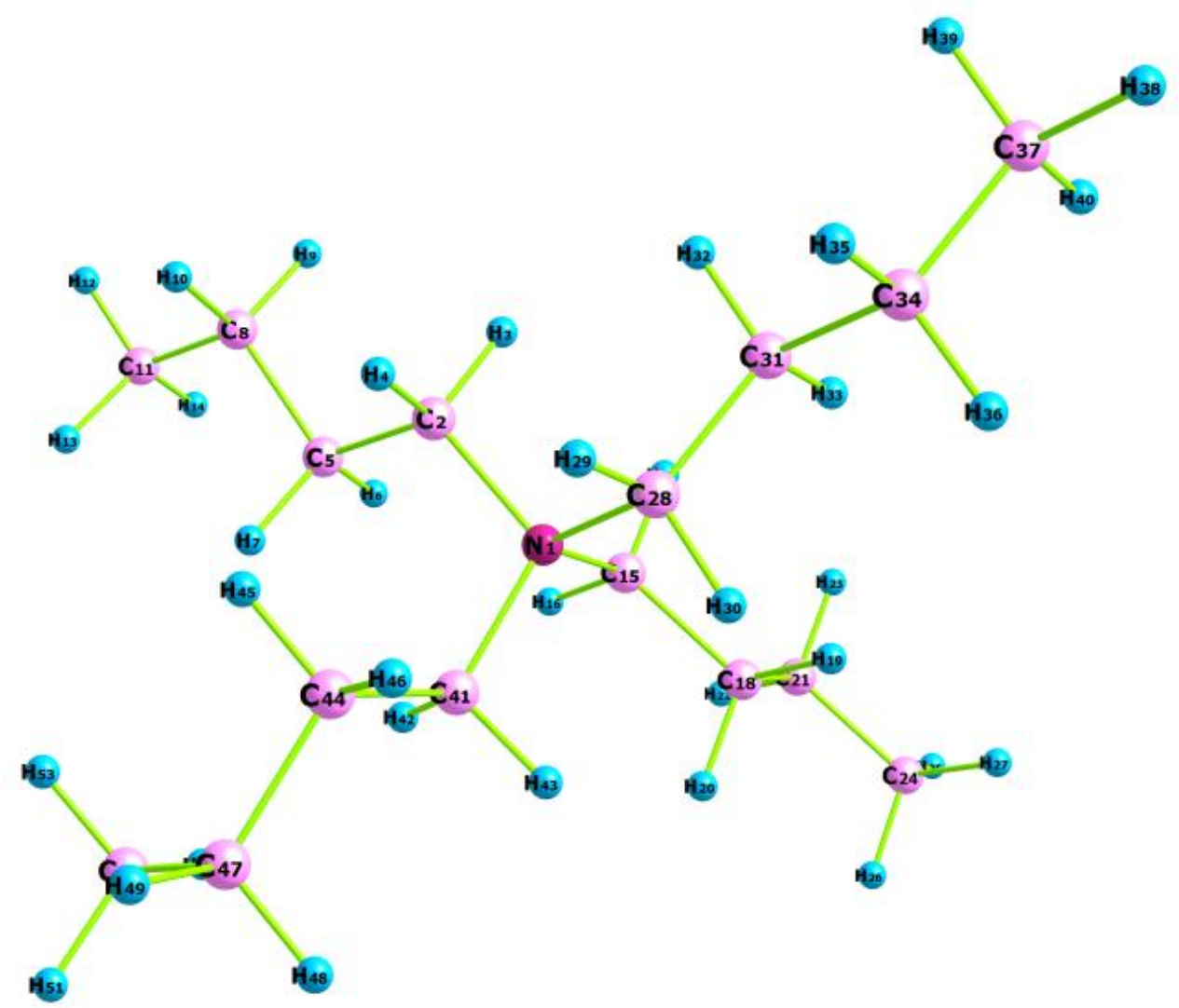

Fig. S17. Structure of the $\left(\left(\mathrm{C}_{4} \mathrm{H}_{9}\right)_{4} \mathrm{~N}\right)^{+}$cation according to the data of quantum calculations

Total Energy

Components:

Nuclear Repulsion

Electronic Energy

One Electron Energy:

Two Electron Energy:
$-685.69732911 \mathrm{Eh}$

$-18658.77292 \mathrm{eV}$

1365.29027352 Eh

$-2050.98760263 \mathrm{Eh}$

$-3616.36906643 \mathrm{Eh}$

1565.38146381 Eh

-1368.21092805 Eh

$682.51359894 \mathrm{Eh}$

2.00466471

$$
\begin{aligned}
& 37151.43709 \mathrm{eV} \\
& -55810.21000 \mathrm{eV} \\
& -98406.40518 \mathrm{eV} \\
& 42596.19518 \mathrm{eV}
\end{aligned}
$$

$-37230.91214 \mathrm{eV}$

$18572.13922 \mathrm{eV}$

Virial Ratio

SCF convergence

Last Energy change

$-1.1105 \mathrm{e}-06$

Tolerance

$1.0000 \mathrm{e}-06$

Last MAX-Density change

7.1897e-05

Tolerance

$1.0000 \mathrm{e}-05$

Last RMS-Density change

$8.0523 \mathrm{e}-07$

Tolerance

$1.0000 \mathrm{e}-06$

Last Orbital Gradient

9.3841e-06

Tolerance

$5.0000 \mathrm{e}-05$

Last Orbital Rotation

$3.0598 \mathrm{e}-05$

Tolerance

$5.0000 \mathrm{e}-05$

Table S2. Energy values and SCF convergence 


\begin{tabular}{|c|c|c|c|c|}
\hline Atom & $\mathbf{x}$ & $\mathbf{y}$ & $\mathbf{z}$ & Charge \\
\hline N1 & 20.10814 & 9.07102 & 8.02703 & 0.233195 \\
\hline C2 & 21.04061 & 9.29750 & 6.84519 & -0.134234 \\
\hline H3 & 20.65030 & 10.16497 & 6.32361 & 0.125540 \\
\hline H4 & 20.91168 & 8.44209 & 6.18874 & 0.117946 \\
\hline C5 & 22.50612 & 9.49640 & 7.16242 & -0.131556 \\
\hline H6 & 22.65342 & 10.33861 & 7.83817 & 0.089306 \\
\hline H7 & 22.92078 & 8.61820 & 7.65444 & 0.074001 \\
\hline C8 & 23.29227 & 9.75366 & 5.87920 & -0.139079 \\
\hline H9 & 22.90144 & 10.64705 & 5.38770 & 0.074438 \\
\hline H10 & 23.12965 & 8.92695 & 5.18384 & 0.082391 \\
\hline C11 & 24.77947 & 9.91802 & 6.13818 & -0.236786 \\
\hline H12 & 25.31902 & 10.09509 & 5.21073 & 0.093553 \\
\hline H13 & 25.20271 & 9.02644 & 6.60027 & 0.073488 \\
\hline H14 & 24.97670 & 10.76049 & 6.80020 & 0.089869 \\
\hline C15 & 20.03418 & 10.35674 & 8.83565 & -0.165915 \\
\hline H16 & 21.04313 & 10.54445 & 9.18836 & 0.123743 \\
\hline H17 & 19.78816 & 11.14201 & 8.12766 & 0.133681 \\
\hline C18 & 19.06768 & 10.37709 & 9.99872 & -0.077883 \\
\hline H19 & 18.05649 & 10.13435 & 9.67356 & 0.077778 \\
\hline H20 & 19.34541 & 9.64057 & 10.75202 & 0.073784 \\
\hline C21 & 19.05694 & 11.76041 & 10.64523 & -0.139202 \\
\hline H22 & 20.06906 & 12.01995 & 10.96366 & 0.071464 \\
\hline H23 & 18.77260 & 12.50518 & 9.89821 & 0.082115 \\
\hline C24 & 18.11171 & 11.83876 & 11.83083 & -0.247398 \\
\hline H25 & 18.12014 & 12.83433 & 12.26857 & 0.099863 \\
\hline H26 & 18.39373 & 11.13384 & 12.61210 & 0.094562 \\
\hline H27 & 17.08600 & 11.61738 & 11.53709 & 0.071855 \\
\hline C28 & 18.73720 & 8.68072 & 7.49718 & -0.110142 \\
\hline H29 & 18.89914 & 7.80640 & 6.87580 & 0.106659 \\
\hline H30 & 18.15886 & 8.35958 & 8.35861 & 0.136114 \\
\hline C31 & 17.98663 & 9.73598 & 6.71589 & -0.188113 \\
\hline H32 & 18.56020 & 10.06221 & 5.84838 & 0.103662 \\
\hline H33 & 17.80269 & 10.61903 & 7.32651 & 0.084562 \\
\hline C34 & 16.64616 & 9.18423 & 6.23675 & -0.116877 \\
\hline H35 & 16.81674 & 8.29225 & 5.63005 & 0.080786 \\
\hline H36 & 16.06043 & 8.85877 & 7.09951 & 0.089402 \\
\hline C37 & 15.85526 & 10.20332 & 5.43573 & -0.231131 \\
\hline H38 & 14.90697 & 9.78607 & 5.10482 & 0.092848 \\
\hline H39 & 16.40168 & 10.52227 & 4.54854 & 0.071107 \\
\hline C40 & 15.63506 & 11.09137 & 6.02733 & 0.080163 \\
\hline & 20.62630 & 7.95965 & 8.92306 & -0.190132 \\
\hline & & & & \\
\hline
\end{tabular}




\begin{tabular}{|c|c|c|c|c|}
\hline H42 & 21.54129 & 8.34088 & 9.36317 & 0.137673 \\
\hline H43 & 19.90143 & 7.85718 & 9.72392 & 0.134192 \\
\hline C44 & 20.85598 & 6.61575 & 8.26553 & -0.094030 \\
\hline H45 & 21.49352 & 6.71455 & 7.38715 & 0.080091 \\
\hline H46 & 19.91048 & 6.19479 & 7.92743 & 0.076312 \\
\hline C47 & 21.51104 & 5.63376 & 9.23922 & -0.128247 \\
\hline H48 & 20.91256 & 5.57021 & 10.15100 & 0.073276 \\
\hline H49 & 21.46466 & 4.64370 & 8.78675 & 0.101695 \\
\hline C50 & 22.95555 & 5.95774 & 9.58273 & -0.297206 \\
\hline H51 & 23.37980 & 5.18726 & 10.22250 & 0.121477 \\
\hline H52 & 23.06195 & 6.90201 & 10.11645 & 0.081046 \\
\hline H53 & 23.57171 & 6.01010 & 8.68493 & 0.094291 \\
\hline
\end{tabular}

Table S3. Coordinates and Mulliken atomic charges 


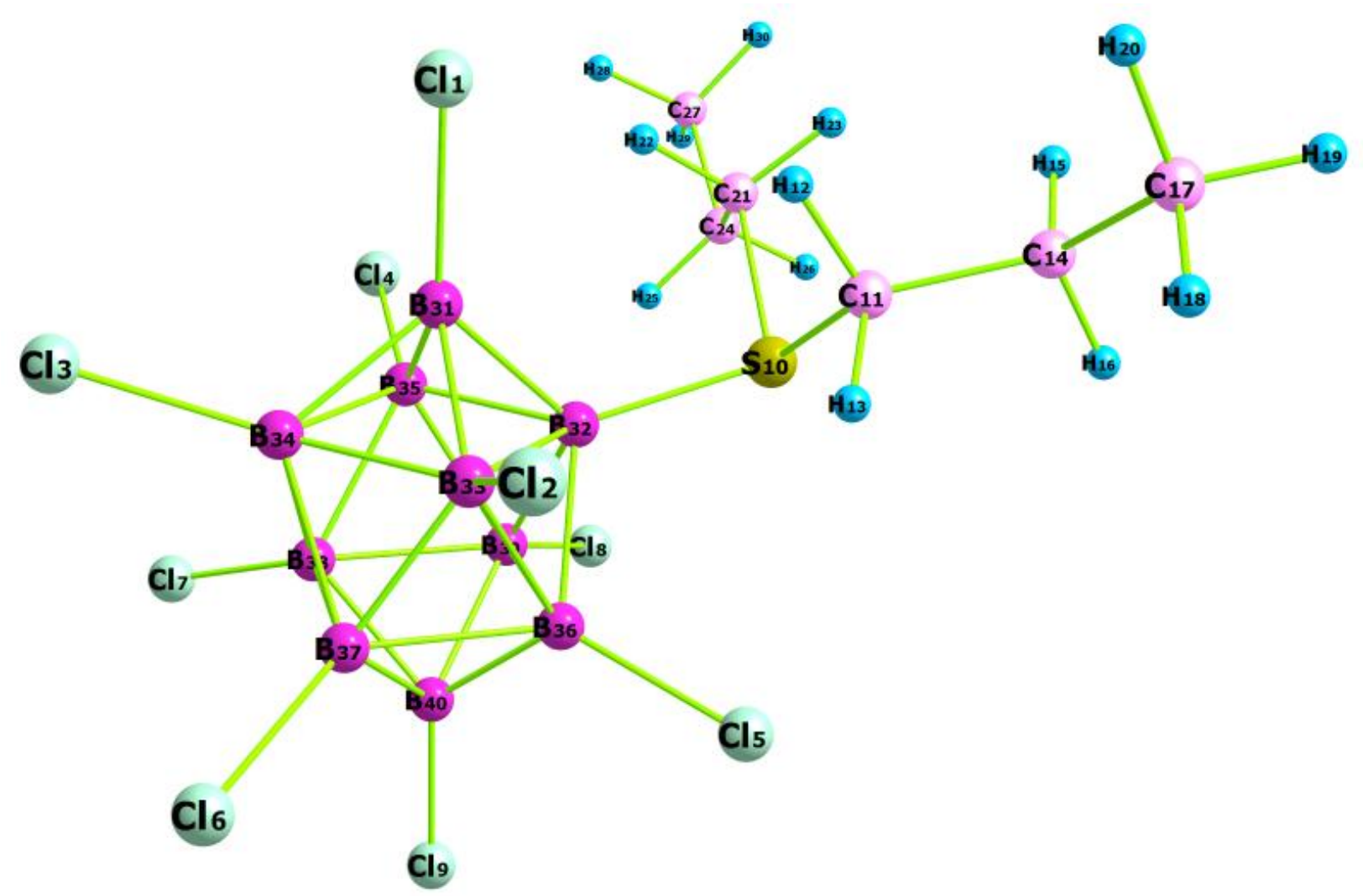

Fig. S18. Structure of the $\left[\mathrm{B}_{10} \mathrm{Cl}{ }_{9} \mathrm{~S}\left(n-\mathrm{C}_{3} \mathrm{H}_{7}\right)_{2}\right]$ cation according to the data of quantum calculations

Total Energy

Components:

Nuclear Repulsion

Electronic Energy

One Electron Energy:

Two Electron Energy:

Virial components:

Potential Energy

Kinetic Energy

Virial Ratio
$-5025.61837787 \mathrm{Eh}$

4417.90715969 Eh

$-9443.52553756 \mathrm{Eh}$

$-15770.59024685 \mathrm{Eh}$

6327.06470929 Eh

$-10043.32697009 \mathrm{Eh}$

$5017.70859222 \mathrm{Eh}$

2.00157637
$-136754.02850 \mathrm{eV}$

$120217.36555 \mathrm{eV}$ $-256971.39405 \mathrm{eV}$ $-429139.57765 \mathrm{eV}$ $172168.18360 \mathrm{eV}$

$-273292.82079 \mathrm{eV}$ $136538.79229 \mathrm{eV}$

SCF convergence

Last Energy change

Last MAX-Density change

$8.9804 \mathrm{e}-08$

Tolerance

$1.0000 \mathrm{e}-06$

Last RMS-Density change

$4.9854 \mathrm{e}-02$

Tolerance

$1.0000 \mathrm{e}-05$

1.3203e-04 Tolerance

$1.0000 \mathrm{e}-06$

Last Orbital Gradient

$9.1736 \mathrm{e}-06$

Tolerance

$5.0000 \mathrm{e}-05$

Last Orbital Rotation

$2.0154 \mathrm{e}-05$

Tolerance

$5.0000 \mathrm{e}-05$

Table S4. Energy values and SCF convergence 


\begin{tabular}{|c|c|c|c|c|}
\hline Atom & $\mathbf{x}$ & $\mathbf{y}$ & $\mathbf{z}$ & Charge \\
\hline $\mathrm{Cl} 1$ & 7.638932 & 2.986460 & 6.419629 & -0.266271 \\
\hline $\mathrm{Cl} 2$ & 10.489986 & 4.710598 & 4.769329 & -0.256297 \\
\hline $\mathrm{Cl} 3$ & 7.329754 & 2.841061 & 2.726842 & -0.218483 \\
\hline $\mathrm{Cl} 4$ & 4.551182 & 4.735739 & 5.265319 & -0.252797 \\
\hline $\mathrm{Cl} 5$ & 9.599489 & 8.185996 & 4.833643 & -0.257559 \\
\hline $\mathrm{Cl} 6$ & 9.361199 & 5.541218 & 1.477703 & -0.240790 \\
\hline $\mathrm{Cl} 7$ & 5.157628 & 5.564323 & 1.801799 & -0.244293 \\
\hline $\mathrm{Cl} 8$ & 5.450788 & 8.220227 & 5.100443 & -0.259967 \\
\hline $\mathrm{Cl} 9$ & 7.294987 & 8.629849 & 1.886584 & -0.226603 \\
\hline $\mathrm{S} 10$ & 7.709355 & 6.757076 & 7.293213 & 0.092252 \\
\hline C11 & 9.291055 & 6.008389 & 7.836985 & -0.128491 \\
\hline $\mathrm{H} 12$ & 9.154202 & 4.930951 & 7.854764 & 0.120787 \\
\hline H13 & 9.980058 & 6.237808 & 7.029693 & 0.124185 \\
\hline $\mathrm{C} 14$ & 9.765732 & 6.579733 & 9.154373 & -0.170346 \\
\hline $\mathrm{H} 15$ & 9.032494 & 6.387008 & 9.940656 & 0.079919 \\
\hline H16 & 9.852993 & 7.663497 & 9.067347 & 0.098991 \\
\hline $\mathrm{C} 17$ & 11.106445 & 5.980056 & 9.551149 & -0.275984 \\
\hline $\mathrm{H} 18$ & 11.862717 & 6.180569 & 8.793610 & 0.115562 \\
\hline H19 & 11.456878 & 6.398296 & 10.493999 & 0.062248 \\
\hline $\mathrm{H} 20$ & 11.037328 & 4.899043 & 9.668211 & 0.102881 \\
\hline $\mathrm{C} 21$ & 6.483170 & 5.805414 & 8.283921 & -0.127387 \\
\hline $\mathrm{H} 22$ & 6.392881 & 4.826112 & 7.826333 & 0.123553 \\
\hline $\mathrm{H} 23$ & 6.930640 & 5.692837 & 9.270143 & 0.104837 \\
\hline $\mathrm{C} 24$ & 5.156334 & 6.529330 & 8.353180 & -0.088653 \\
\hline $\mathrm{H} 25$ & 4.808862 & 6.744370 & 7.346139 & 0.074225 \\
\hline $\mathrm{H} 26$ & 5.285041 & 7.491100 & 8.853114 & 0.074944 \\
\hline $\mathrm{C} 27$ & 4.123842 & 5.680832 & 9.075672 & -0.265222 \\
\hline $\mathrm{H} 28$ & 3.958757 & 4.745133 & 8.543922 & 0.095202 \\
\hline $\mathrm{H} 29$ & 3.169530 & 6.202468 & 9.131835 & 0.082762 \\
\hline $\mathrm{H} 30$ & 4.433459 & 5.441440 & 10.094777 & 0.052658 \\
\hline B31 & 7.539164 & 4.378902 & 5.286895 & 0.378719 \\
\hline B32 & 7.559115 & 6.012983 & 5.556748 & -0.067044 \\
\hline B33 & 8.769763 & 5.199407 & 4.497895 & 0.047208 \\
\hline B34 & 7.405623 & 4.399435 & 3.615898 & -0.003989 \\
\hline B35 & 6.202916 & 5.211856 & 4.700670 & -0.006539 \\
\hline B36 & 8.380138 & 6.954884 & 4.289294 & -0.157497 \\
\hline B37 & 8.270983 & 5.816393 & 2.880817 & 0.061210 \\
\hline B38 & 6.453546 & 5.825059 & 3.020082 & 0.051303 \\
\hline B39 & 6.566978 & 6.963037 & 4.423723 & -0.026167 \\
\hline B40 & 7.373765 & 7.230169 & 2.985891 & 0.596931 \\
\hline
\end{tabular}

Table S5. Coordinates and Mulliken atomic charges 


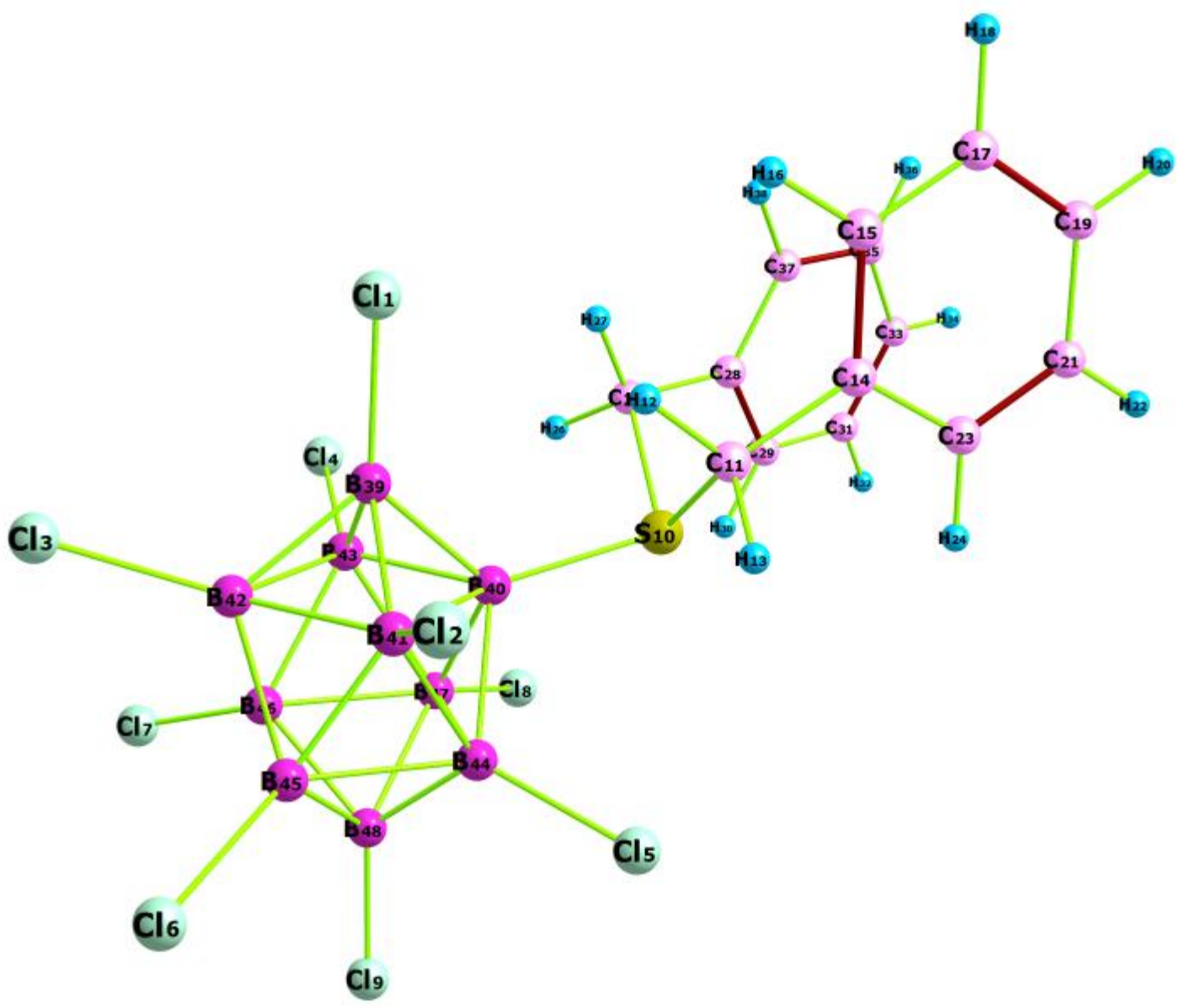

Fig. S19. Structure of the $\left[\mathrm{B}_{10} \mathrm{Cl}_{9} \mathrm{~S}\left(\mathrm{CH}_{2} \mathrm{Ph}\right)_{2}\right]$ cation according to the data of quantum calculations

Total Energy

Components:

Nuclear Repulsion

Electronic Energy

One Electron Energy:

Two Electron Energy:

Virial components:

Potential Energy

Kinetic Energy

Virial Ratio
$-5330.38874720 \mathrm{Eh}$

5712.66294026 Eh $-11043.05168746 \mathrm{Eh}$ $-18766.71739942 \mathrm{Eh}$ 7723.66571196 Eh

-10651.62481385 Eh $5321.23606664 \mathrm{Eh}$ 2.00172003
$-145047.25187 \mathrm{eV}$

$155449.46150 \mathrm{eV}$ $-300496.71337 \mathrm{eV}$ $-510668.34231 \mathrm{eV}$ $210171.62894 \mathrm{eV}$

$-289845.44664 \mathrm{eV}$ $144798.19477 \mathrm{eV}$

SCF convergence

Last Energy change

Last MAX-Density change

Last RMS-Density change

Last Orbital Gradient

Last Orbital Rotation

$-4.0508 \mathrm{e}-07$

7.6161e-02

$1.5394 \mathrm{e}-04$

$1.1266 \mathrm{e}-05$

$2.9484 \mathrm{e}-05$
Tolerance

Tolerance

Tolerance

Tolerance

Tolerance
$1.0000 \mathrm{e}-06$

$1.0000 \mathrm{e}-05$

$1.0000 \mathrm{e}-06$

$5.0000 \mathrm{e}-05$

$5.0000 \mathrm{e}-05$

Table S6. Energy values and SCF convergence 


\begin{tabular}{|c|c|c|c|c|}
\hline Atom & $\mathbf{x}$ & $\mathbf{y}$ & $\mathbf{z}$ & Charge \\
\hline C11 & 2.93773 & 4.57380 & 1.61190 & -0.266088 \\
\hline C12 & 3.98474 & 7.01180 & 4.22171 & -0.243433 \\
\hline C13 & 0.31415 & 4.94057 & 4.25270 & -0.228789 \\
\hline C14 & 2.34159 & 1.34411 & 3.38621 & -0.245458 \\
\hline C15 & 6.10161 & 5.12364 & 6.40490 & -0.258827 \\
\hline C16 & 2.03230 & 6.22272 & 7.14930 & -0.239094 \\
\hline C17 & 0.87063 & 2.22275 & 6.55291 & -0.240517 \\
\hline C18 & 4.95709 & 1.17442 & 5.81103 & -0.266223 \\
\hline C19 & 3.73958 & 3.28401 & 8.70028 & -0.217464 \\
\hline S10 & 6.08009 & 3.37918 & 3.50706 & 0.074825 \\
\hline C11 & 6.41814 & 4.95611 & 2.61202 & -0.095644 \\
\hline H12 & 5.48583 & 5.27365 & 2.16001 & 0.122521 \\
\hline H13 & 6.65510 & 5.64103 & 3.42337 & 0.161301 \\
\hline C14 & 7.51772 & 4.81248 & 1.61934 & -0.051655 \\
\hline C15 & 7.26958 & 5.07885 & 0.27660 & -0.119329 \\
\hline H16 & 6.27721 & 5.38597 & -0.02753 & 0.120900 \\
\hline C17 & 8.27938 & 4.95078 & -0.66661 & -0.123740 \\
\hline H18 & 8.07222 & 5.16406 & -1.70716 & 0.121087 \\
\hline C19 & 9.54494 & 4.54023 & -0.27731 & -0.151063 \\
\hline H20 & 10.33067 & 4.42703 & -1.01296 & 0.124841 \\
\hline C21 & 9.79968 & 4.26881 & 1.06107 & -0.084219 \\
\hline H22 & 10.78331 & 3.94150 & 1.37039 & 0.110410 \\
\hline C23 & 8.79565 & 4.40955 & 2.00286 & -0.176307 \\
\hline H24 & 8.99450 & 4.18842 & 3.04337 & 0.142831 \\
\hline C25 & 5.63734 & 2.20191 & 2.15467 & -0.066957 \\
\hline H26 & 4.91930 & 1.54142 & 2.63398 & 0.144880 \\
\hline H27 & 5.11464 & 2.75943 & 1.38595 & 0.109942 \\
\hline C28 & 6.84097 & 1.47340 & 1.66296 & -0.025908 \\
\hline C29 & 7.56656 & 0.65360 & 2.52682 & -0.133158 \\
\hline H30 & 7.24696 & 0.55405 & 3.55623 & 0.122095 \\
\hline C31 & 8.69043 & -0.01853 & 2.07726 & -0.146148 \\
\hline H32 & 9.24499 & -0.65091 & 2.75811 & 0.131676 \\
\hline C33 & 9.10178 & 0.11334 & 0.75656 & -0.120265 \\
\hline H34 & 9.98253 & -0.40976 & 0.40674 & 0.109143 \\
\hline C35 & 8.37919 & 0.91911 & -0.11009 & -0.135610 \\
\hline H36 & 8.69510 & 1.03350 & -1.13860 & 0.113437 \\
\hline C37 & 7.25571 & 1.59464 & 0.34195 & -0.110308 \\
\hline H38 & 6.70677 & 2.23820 & -0.33115 & 0.123415 \\
\hline & 3.09848 & 4.26139 & 3.37622 & 0.367466 \\
\hline B40 & 4.39694 & 3.75425 & 4.26518 & 0.039521 \\
\hline & 3.55696 & 5.30054 & 4.61335 & -0.006072 \\
\hline & 1.98548 & 4.40006 & 4.62312 & 0.015973 \\
\hline & & & & \\
\hline
\end{tabular}




\begin{tabular}{|c|c|c|c|c|}
\hline B43 & 2.84571 & 2.85075 & 4.25036 & 0.059155 \\
\hline B44 & 4.49903 & 4.43693 & 5.89823 & -0.152244 \\
\hline B45 & 2.77080 & 4.89777 & 6.18540 & 0.109946 \\
\hline B46 & 2.26753 & 3.16519 & 5.92775 & 0.021251 \\
\hline B47 & 3.99725 & 2.70790 & 5.64133 & -0.048609 \\
\hline B48 & 3.51702 & 3.60674 & 6.96272 & 0.506510 \\
\hline
\end{tabular}

Table S7. Coordinates and Mulliken atomic charges 


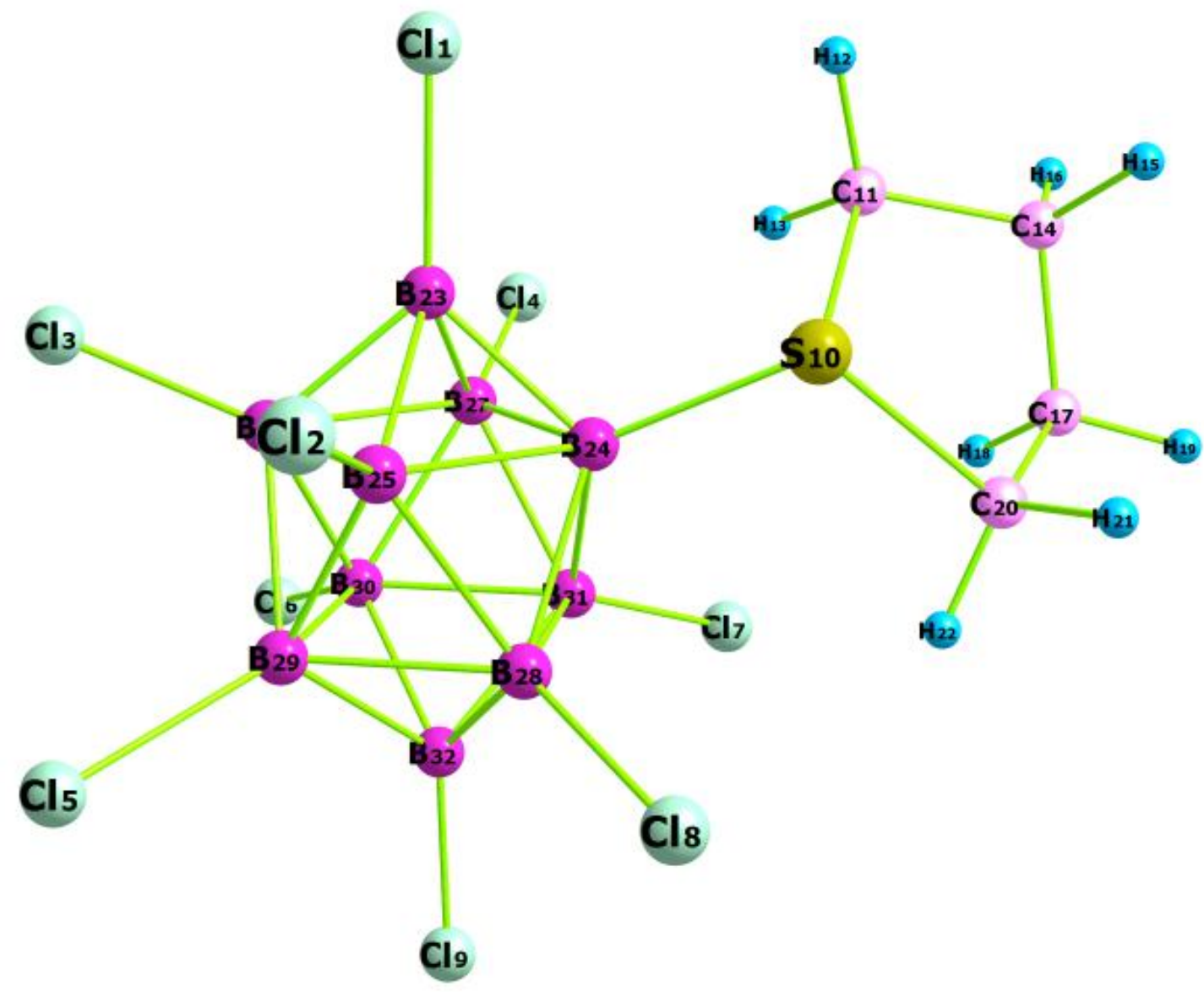

Fig. S20. Structure of the $\left[\mathrm{B}_{10} \mathrm{Cl}_{9}-c y c l o-\mathrm{S}\left(\mathrm{C}_{4} \mathrm{H}_{8}\right)\right]$ cation according to the data of quantum calculations

Total Energy

Components:

Nuclear Repulsion

Electronic Energy

One Electron Energy:

Two Electron Energy:

Virial components:

Potential Energy

Kinetic Energy

Virial Ratio

SCF convergence

Last Energy change

Last MAX-Density change

Last RMS-Density change

Last Orbital Gradient

Last Orbital Rotation
$-4945.81541478 \mathrm{Eh}$

$-134582.47948 \mathrm{eV}$

3973.14995152 Eh

$-8918.96536630 \mathrm{Eh}$

$-14775.20919008 \mathrm{Eh}$

5856.24382378 Eh

$108114.90664 \mathrm{eV}$ $-242697.38611 \mathrm{eV}$ $-402053.88209 \mathrm{eV}$ $159356.49597 \mathrm{eV}$

$-9884.11990582 \mathrm{Eh}$ $-268960.57633 \mathrm{eV}$ 4938.30449103 Eh 2.00152095

Table S8. Energy values and SCF convergence

$1.0000 \mathrm{e}-06$

$1.0000 \mathrm{e}-05$

$1.0000 \mathrm{e}-06$

$5.0000 \mathrm{e}-05$

$5.0000 \mathrm{e}-05$

$\begin{array}{lll}-1.9781 \mathrm{e}-07 & \text { Tolerance } & 1.0000 \mathrm{e}-06 \\ 4.3534 \mathrm{e}-02 & \text { Tolerance } & 1.0000 \mathrm{e}-05 \\ 1.2909 \mathrm{e}-04 & \text { Tolerance } & 1.0000 \mathrm{e}-06 \\ 6.7441 \mathrm{e}-06 & \text { Tolerance } & 5.0000 \mathrm{e}-05 \\ 2.2825 \mathrm{e}-05 & \text { Tolerance } & 5.0000 \mathrm{e}-05\end{array}$




\begin{tabular}{|c|c|c|c|c|}
\hline Atom & $\mathbf{x}$ & $\mathbf{y}$ & $\mathbf{z}$ & Charge \\
\hline C11 & -0.731353 & -5.022594 & 9.204423 & -0.242886 \\
\hline C12 & -0.397101 & -1.415907 & 9.980901 & -0.231350 \\
\hline C13 & 0.266462 & -4.529112 & 12.781417 & -0.231341 \\
\hline C14 & 2.595228 & -6.561038 & 9.905212 & -0.262579 \\
\hline C15 & 1.723295 & -1.229193 & 12.907972 & -0.246500 \\
\hline C16 & 3.850901 & -4.877375 & 12.832653 & -0.236965 \\
\hline C17 & 5.020287 & -4.153034 & 8.894563 & -0.278716 \\
\hline C18 & 2.878734 & -0.517510 & 8.861478 & -0.260018 \\
\hline C19 & 5.202963 & -1.625065 & 11.579364 & -0.230509 \\
\hline S10 & 1.667411 & -3.339447 & 7.184211 & 0.104834 \\
\hline C11 & 1.646774 & -5.094857 & 6.609712 & -0.137526 \\
\hline H12 & 0.608955 & -5.379437 & 6.484552 & 0.128294 \\
\hline H13 & 2.088055 & -5.697345 & 7.396229 & 0.132623 \\
\hline C14 & 2.473320 & -5.087177 & 5.342012 & -0.109551 \\
\hline H15 & 1.895464 & -4.673637 & 4.513153 & 0.070143 \\
\hline H16 & 2.748909 & -6.106821 & 5.073875 & 0.063150 \\
\hline C17 & 3.696798 & -4.225831 & 5.622878 & -0.123545 \\
\hline H18 & 4.351971 & -4.737442 & 6.323092 & 0.096258 \\
\hline H19 & 4.265465 & -4.018609 & 4.716299 & -0.179068 \\
\hline C20 & 3.220791 & -2.930797 & 6.255079 & 0.130764 \\
\hline H21 & 2.921007 & -2.184500 & 5.524292 & 0.150426 \\
\hline H22 & 3.920799 & -2.488287 & 6.951332 & 0.320327 \\
\hline B23 & 0.727362 & -4.194022 & 9.840264 & -0.087873 \\
\hline B24 & 1.970229 & -3.451929 & 9.027462 & 0.048694 \\
\hline B25 & 0.957780 & -2.577424 & 10.228903 & -0.043728 \\
\hline B26 & 1.250784 & -3.920277 & 11.409299 & 0.085330 \\
\hline B27 & 2.255140 & -4.804498 & 10.179246 & -0.096295 \\
\hline B28 & 2.642030 & -2.027125 & 9.842261 & 0.036092 \\
\hline B29 & 2.142578 & -2.343109 & 11.560637 & 0.024195 \\
\hline B30 & 3.058827 & -3.919903 & 11.533078 & -0.024845 \\
\hline B31 & 3.557878 & -3.608733 & 9.823739 & 0.564928 \\
\hline B32 & 3.730308 & -2.465172 & 11.030383 & 0.067238 \\
\hline & Tab1e S9. Co0r & & \\
\hline & & & & \\
\hline
\end{tabular}

Table S9. Coordinates and Mulliken atomic charges 
NMR and IR data

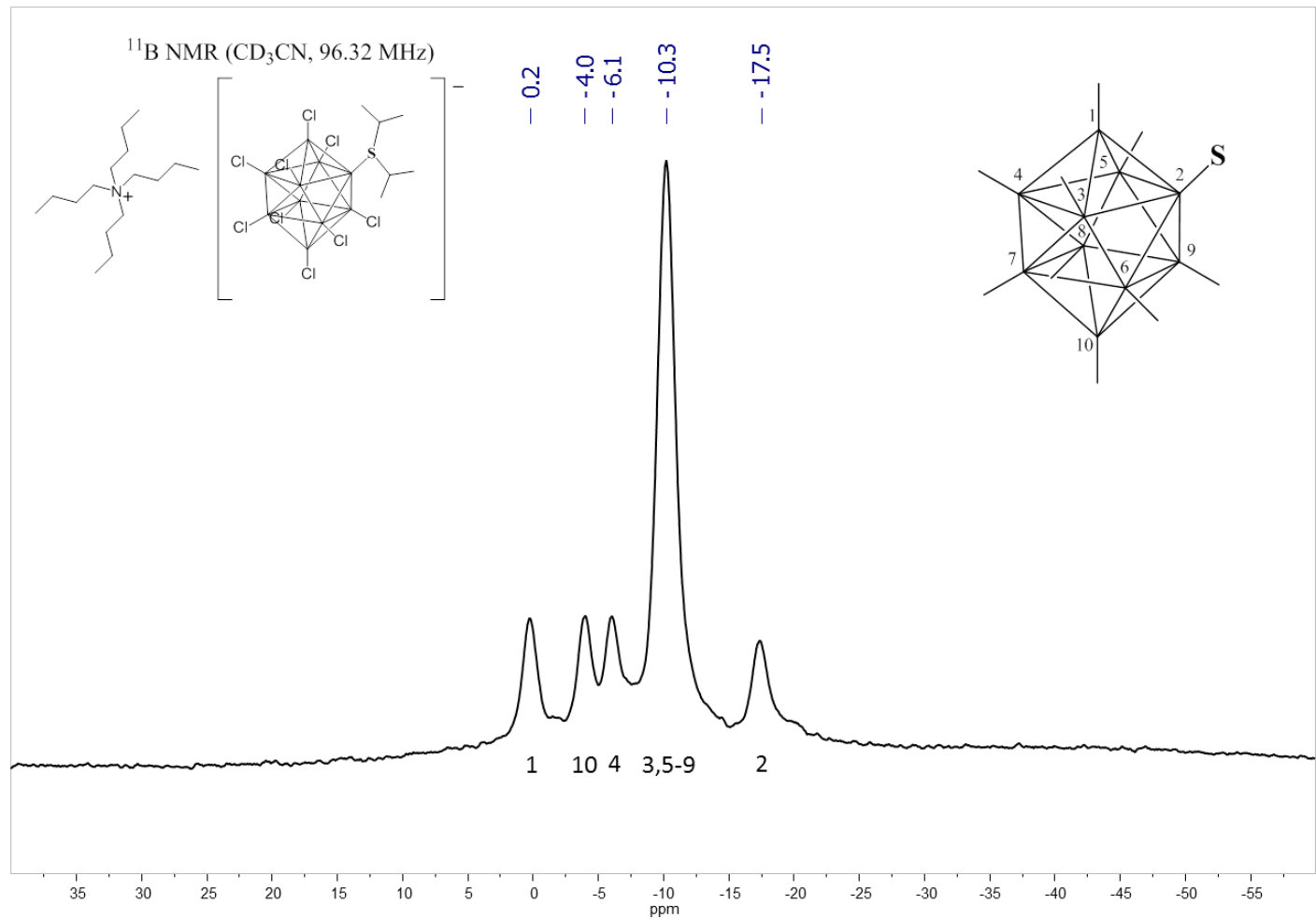

Fig. S21. ${ }^{11}$ B NMR spectrum (acetonitrile-d3, 96.32 MHz) of $\left(\left(\mathrm{C}_{4} \mathrm{H}_{9}\right)_{4} \mathrm{~N}\right)\left[\mathrm{B}_{10} \mathrm{Cl} 9 \mathrm{~S}\left(i-\mathrm{C}_{3} \mathrm{H}_{7}\right)_{2}\right]$

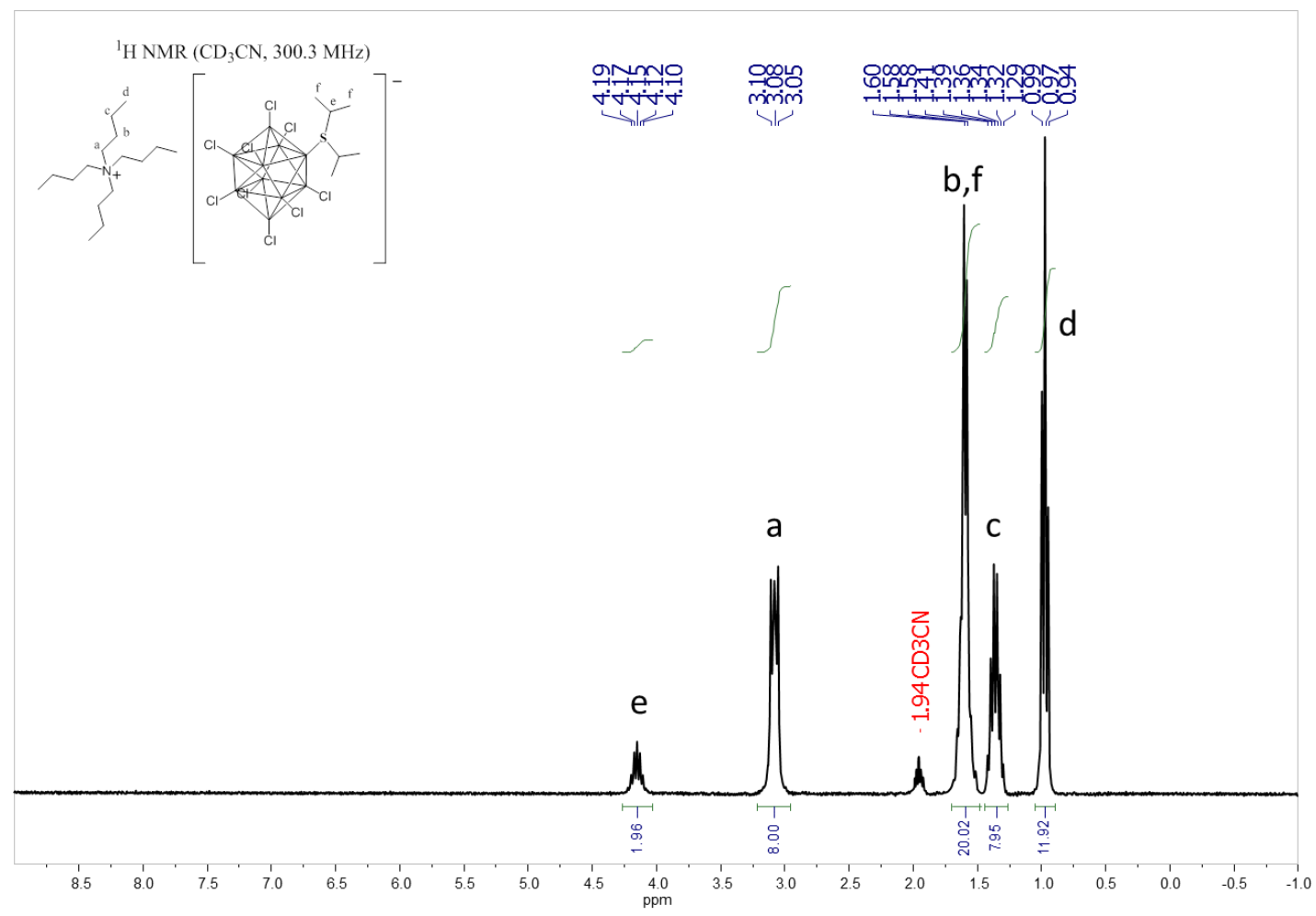

Fig. S22. ${ }^{1} \mathrm{H}$ NMR spectrum (acetonitrile-d3, 300.3 MHz) of $\left(\left(\mathrm{C}_{4} \mathrm{H}_{9}\right)_{4} \mathrm{~N}\right)\left[\mathrm{B}_{10} \mathrm{Cl}_{9} \mathrm{~S}\left(i-\mathrm{C}_{3} \mathrm{H}_{7}\right)_{2}\right]$ 


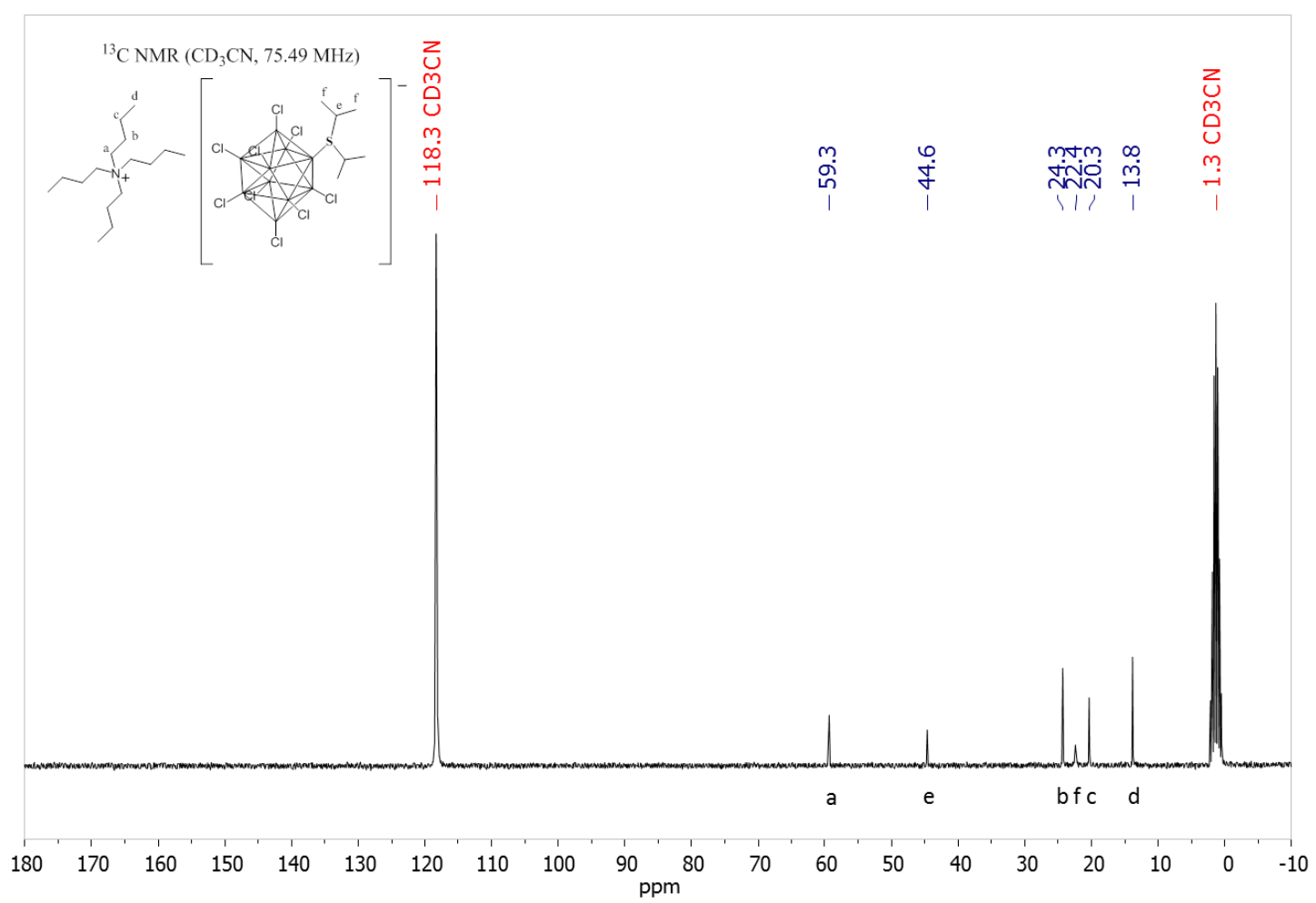

Fig. S23. ${ }^{13} \mathrm{C}$ NMR spectrum (acetonitrile-d3, 75.49 MHz) of $\left(\left(\mathrm{C}_{4} \mathrm{H}_{9}\right)_{4} \mathrm{~N}\right)\left[\mathrm{B}_{10} \mathrm{Cl}_{9} \mathrm{~S}\left(i-\mathrm{C}_{3} \mathrm{H}_{7}\right)_{2}\right]$

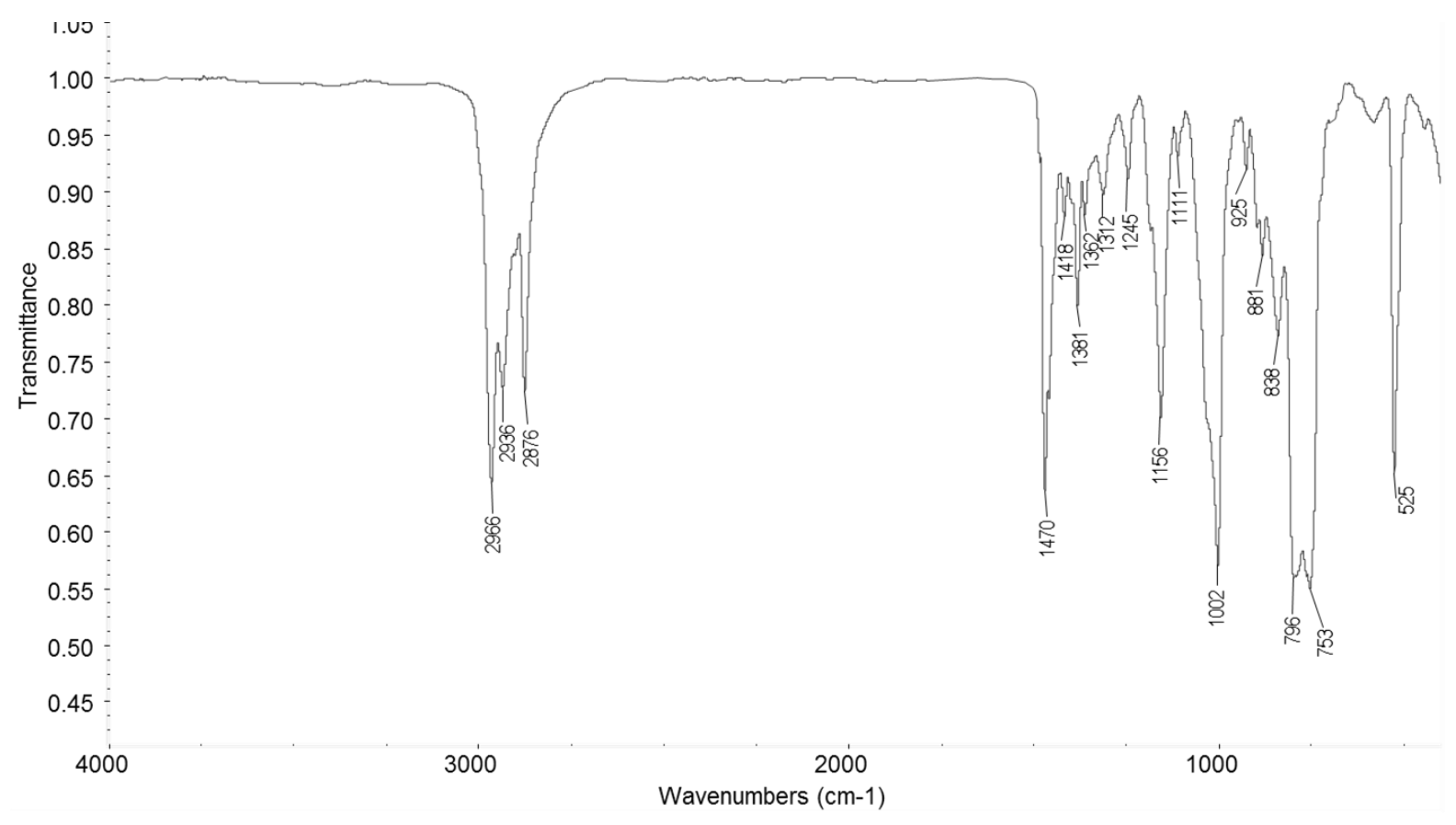

Fig. S24. IR spectra (in $\left.\mathrm{CCl}_{4}\right)$ of $\left(\left(\mathrm{C}_{4} \mathrm{H}_{9}\right)_{4} \mathrm{~N}\right)\left[\mathrm{B}_{10} \mathrm{Cl}_{9} \mathrm{~S}\left(i-\mathrm{C}_{3} \mathrm{H}_{7}\right)_{2}\right]$ 


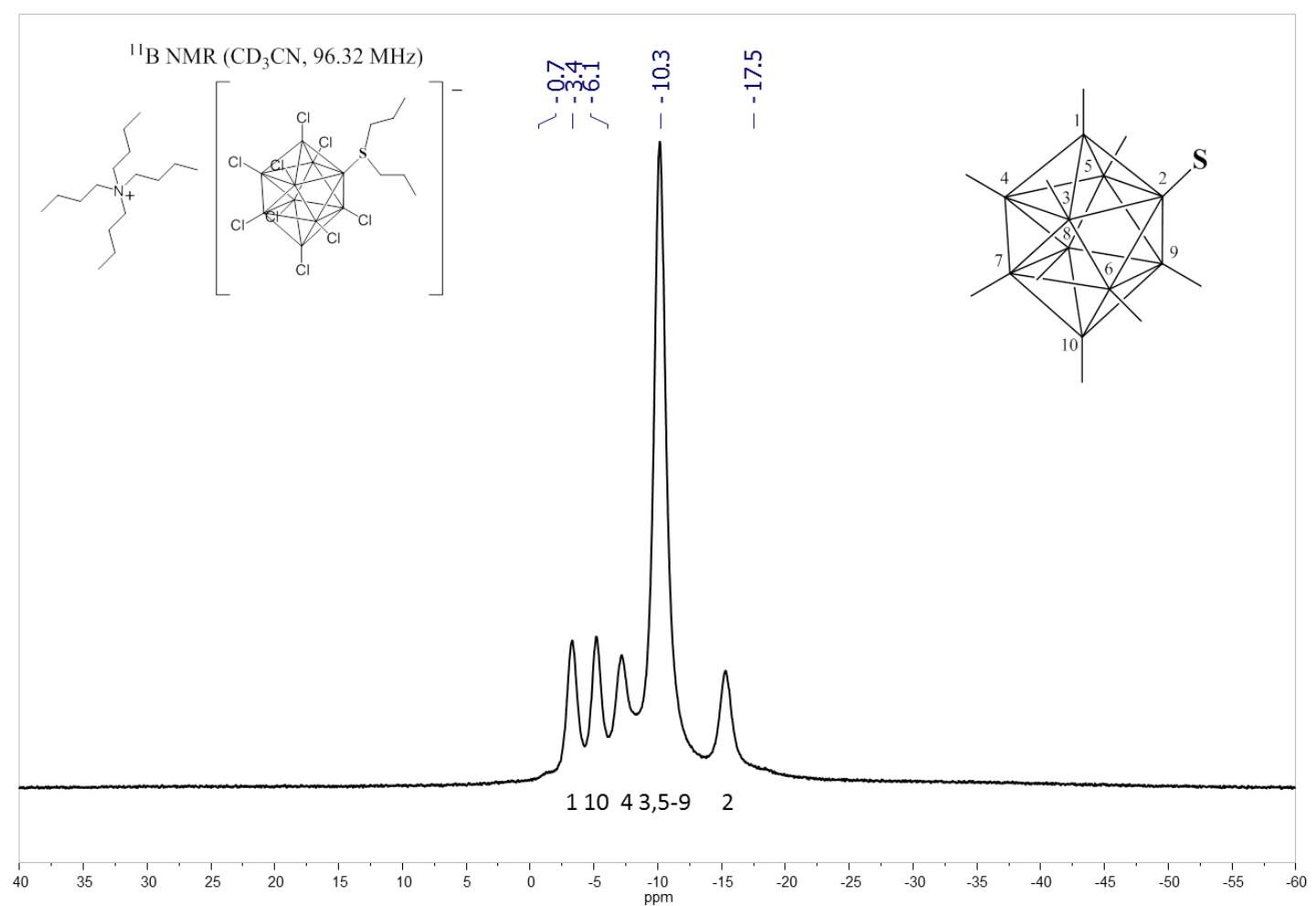

Fig. S25. ${ }^{11} \mathrm{~B}$ NMR spectrum (acetonitrile-d3, 96.32 MHz) of $\left(\left(\mathrm{C}_{4} \mathrm{H}_{9}\right)_{4} \mathrm{~N}\right)\left[\mathrm{B}_{10} \mathrm{Cl}_{9} \mathrm{~S}\left(n-\mathrm{C}_{3} \mathrm{H}_{7}\right)_{2}\right]$

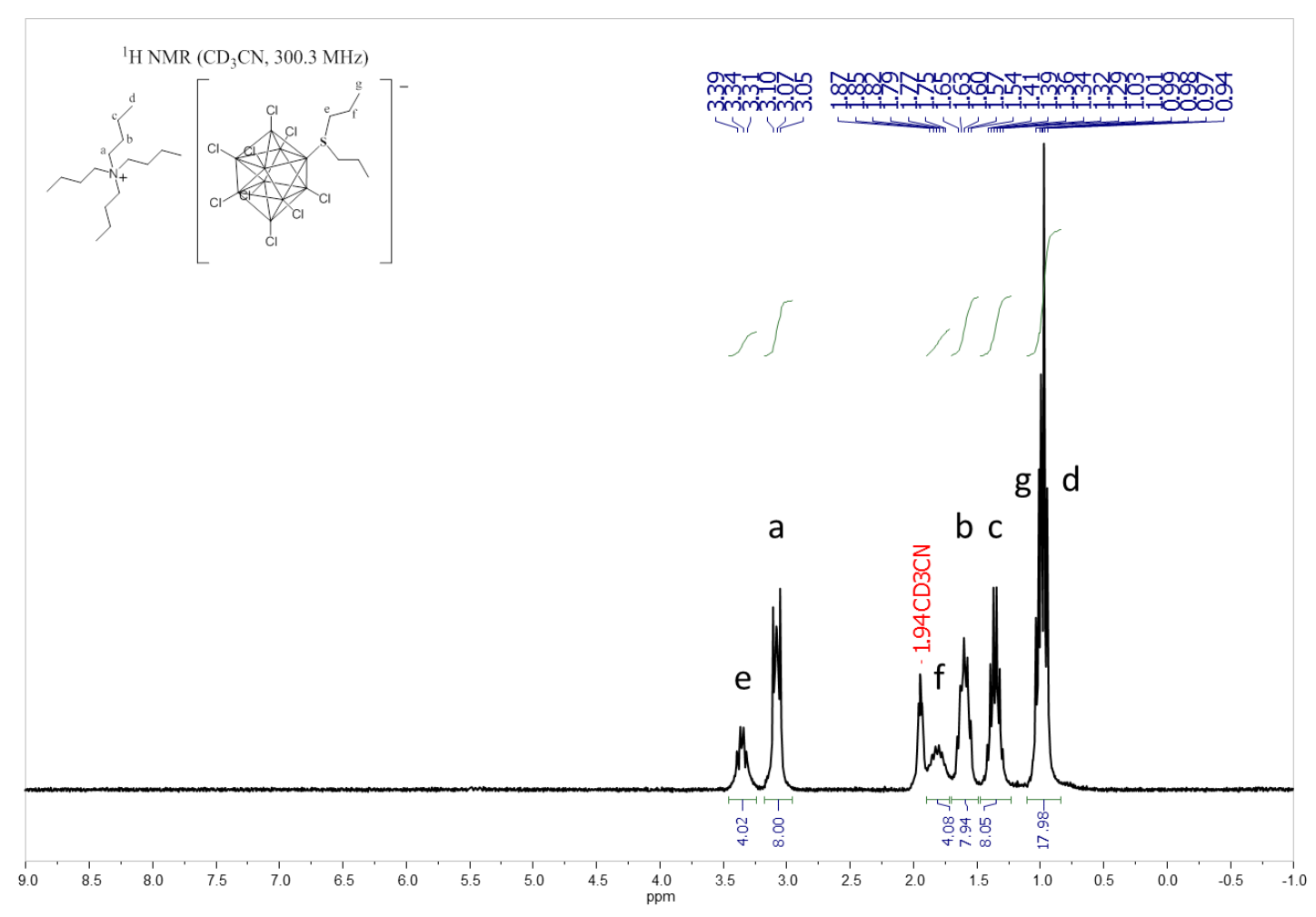

Fig. S26. ${ }^{1} \mathrm{H}$ NMR spectrum (acetonitrile-d3, 300.3 MHz) of $\left(\left(\mathrm{C}_{4} \mathrm{H}_{9}\right)_{4} \mathrm{~N}\right)\left[\mathrm{B}_{10} \mathrm{Cl}_{9} \mathrm{~S}\left(n-\mathrm{C}_{3} \mathrm{H}_{7}\right)_{2}\right]$ 


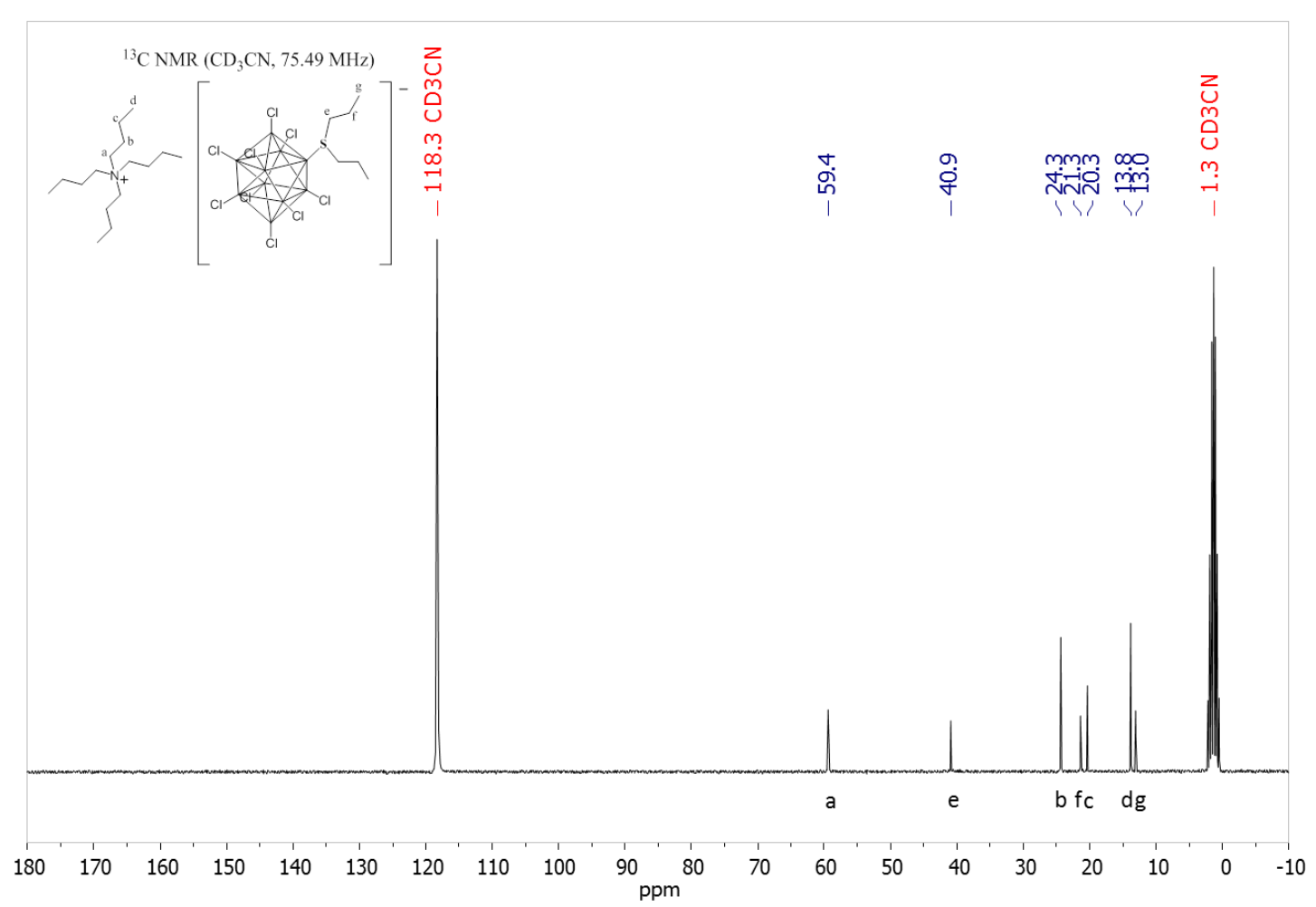

Fig. S27. ${ }^{13} \mathrm{C}$ NMR spectrum (acetonitrile-d3, 75.49 MHz) of $\left(\left(\mathrm{C}_{4} \mathrm{H}_{9}\right)_{4} \mathrm{~N}\right)\left[\mathrm{B}_{10} \mathrm{Cl}_{9} \mathrm{~S}\left(n-\mathrm{C}_{3} \mathrm{H}_{7}\right)_{2}\right]$

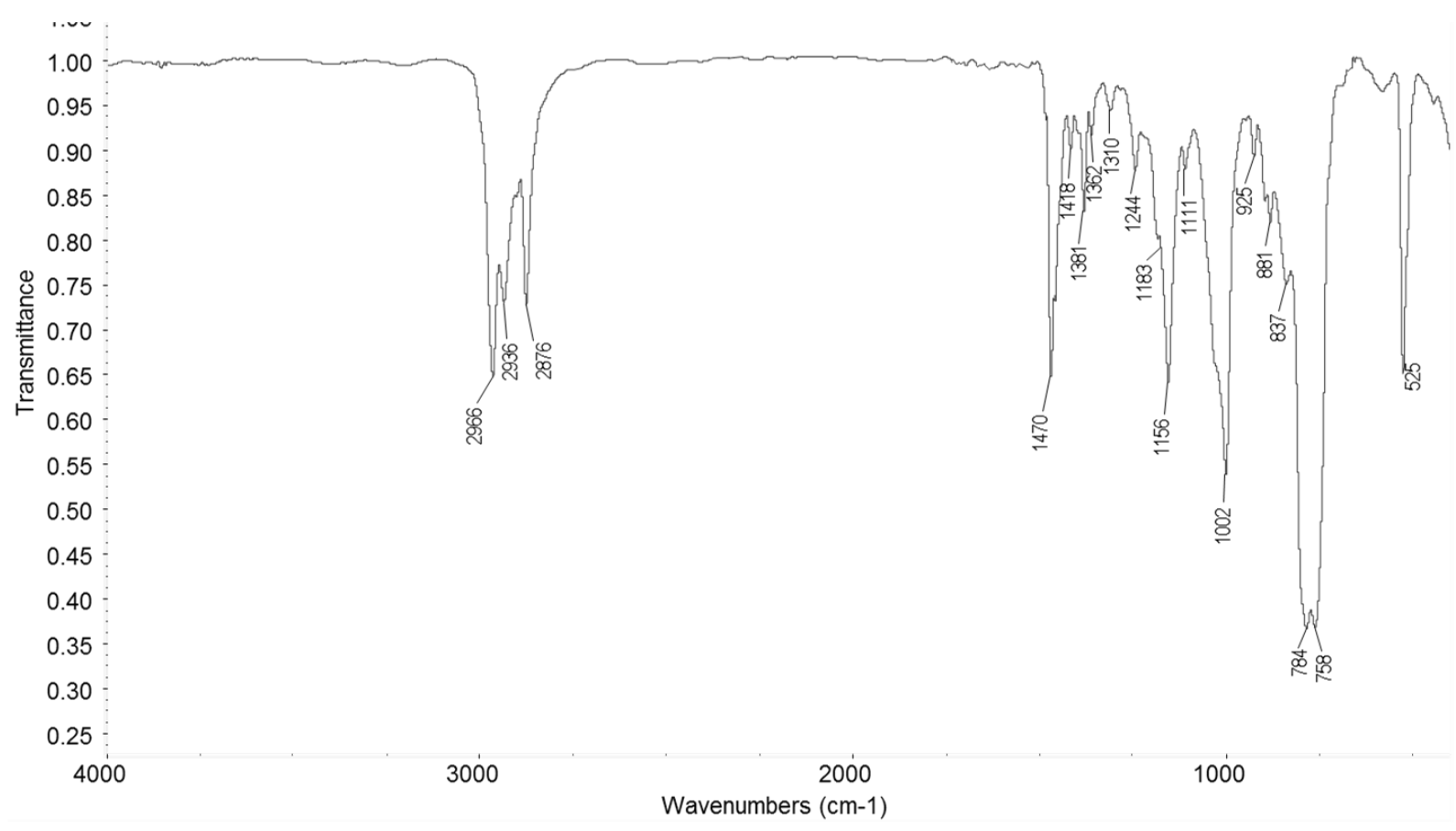

Fig. S28. IR spectra (in $\left.\mathrm{CCl}_{4}\right)$ of $\left(\left(\mathrm{C}_{4} \mathrm{H}_{9}\right)_{4} \mathrm{~N}\right)\left[\mathrm{B}_{10} \mathrm{Cl}_{9} \mathrm{~S}\left(n-\mathrm{C}_{3} \mathrm{H}_{7}\right)_{2}\right]$ 


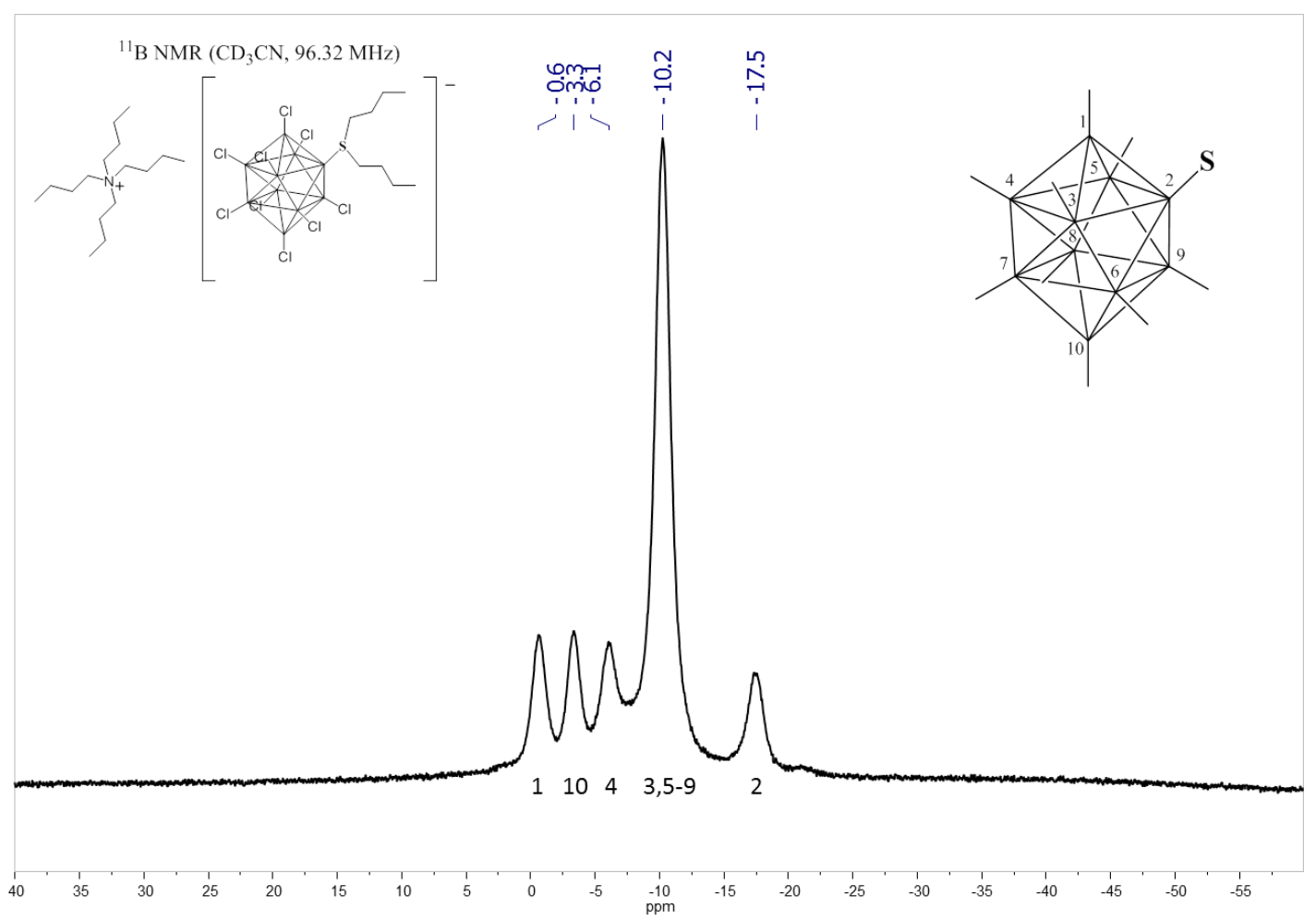

Fig. S29. ${ }^{11}$ B NMR spectrum (acetonitrile-d3, 96.32 MHz) of $\left(\left(\mathrm{C}_{4} \mathrm{H}_{9}\right)_{4} \mathrm{~N}\right)\left[\mathrm{B}_{10} \mathrm{Cl}{ }_{9} \mathrm{~S}\left(n-\mathrm{C}_{4} \mathrm{H}_{9}\right)_{2}\right]$

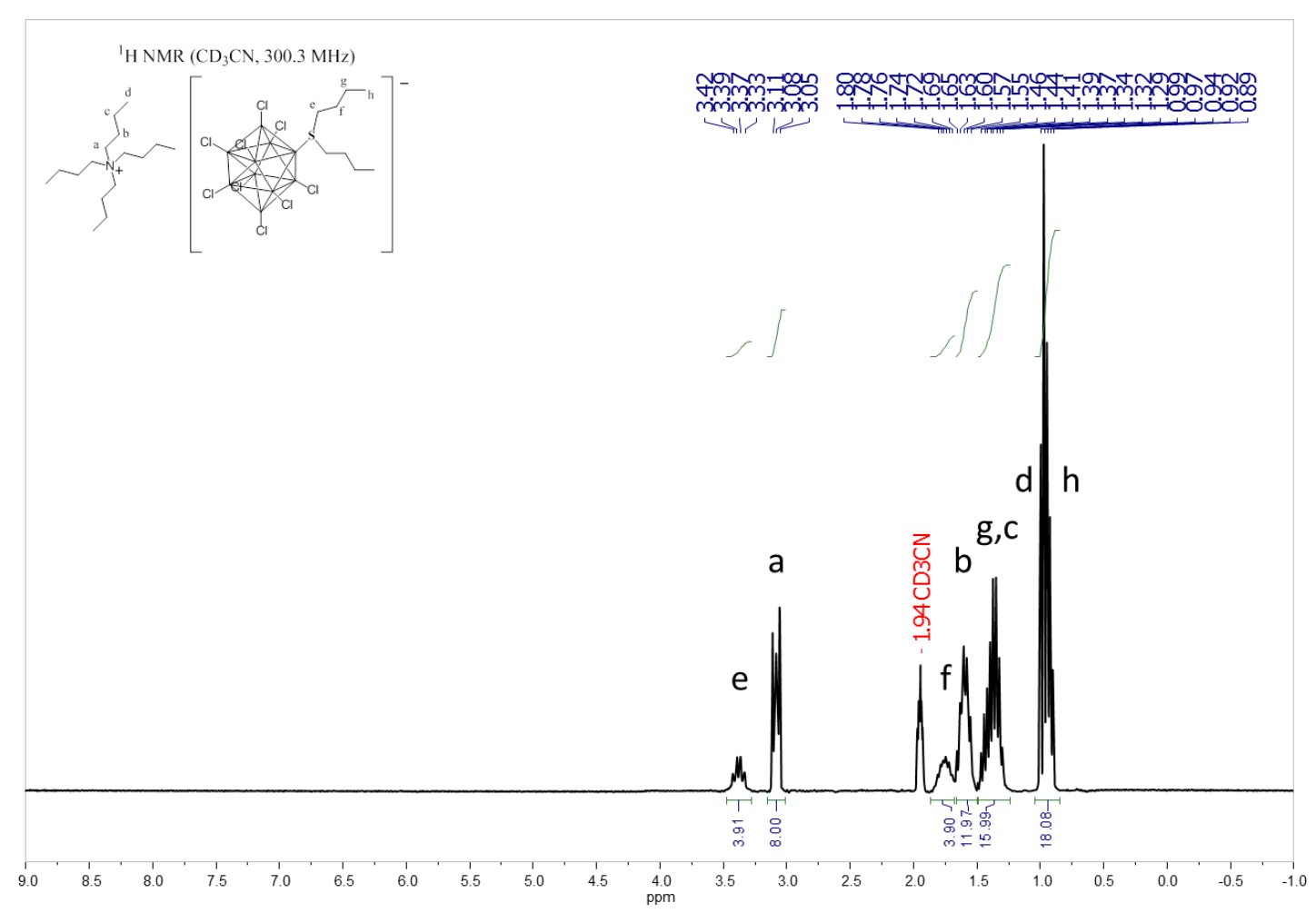

Fig. S30. ${ }^{1} \mathrm{H}$ NMR spectrum (acetonitrile-d3, 300.3 MHz) of $\left(\left(\mathrm{C}_{4} \mathrm{H}_{9}\right)_{4} \mathrm{~N}\right)\left[\mathrm{B}_{10} \mathrm{Cl}_{9} \mathrm{~S}\left(n-\mathrm{C}_{4} \mathrm{H}_{9}\right)_{2}\right]$ 


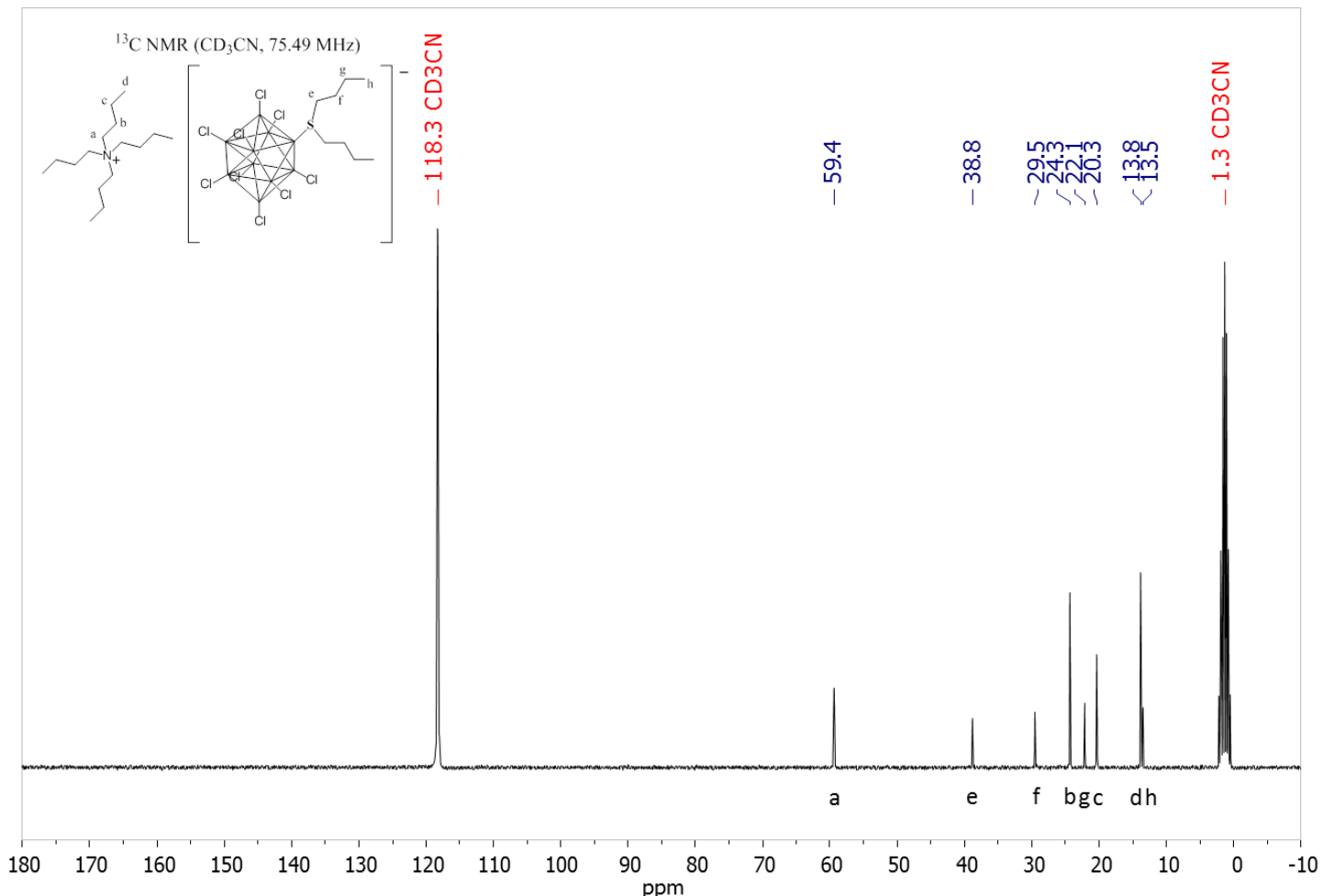

Fig. S31. ${ }^{13} \mathrm{C}$ NMR spectrum (acetonitrile-d3, 75.49 MHz) of $\left(\left(\mathrm{C}_{4} \mathrm{H}_{9}\right)_{4} \mathrm{~N}\right)\left[\mathrm{B}_{10} \mathrm{Cl}{ }_{9} \mathrm{~S}\left(n-\mathrm{C}_{4} \mathrm{H}_{9}\right)_{2}\right]$

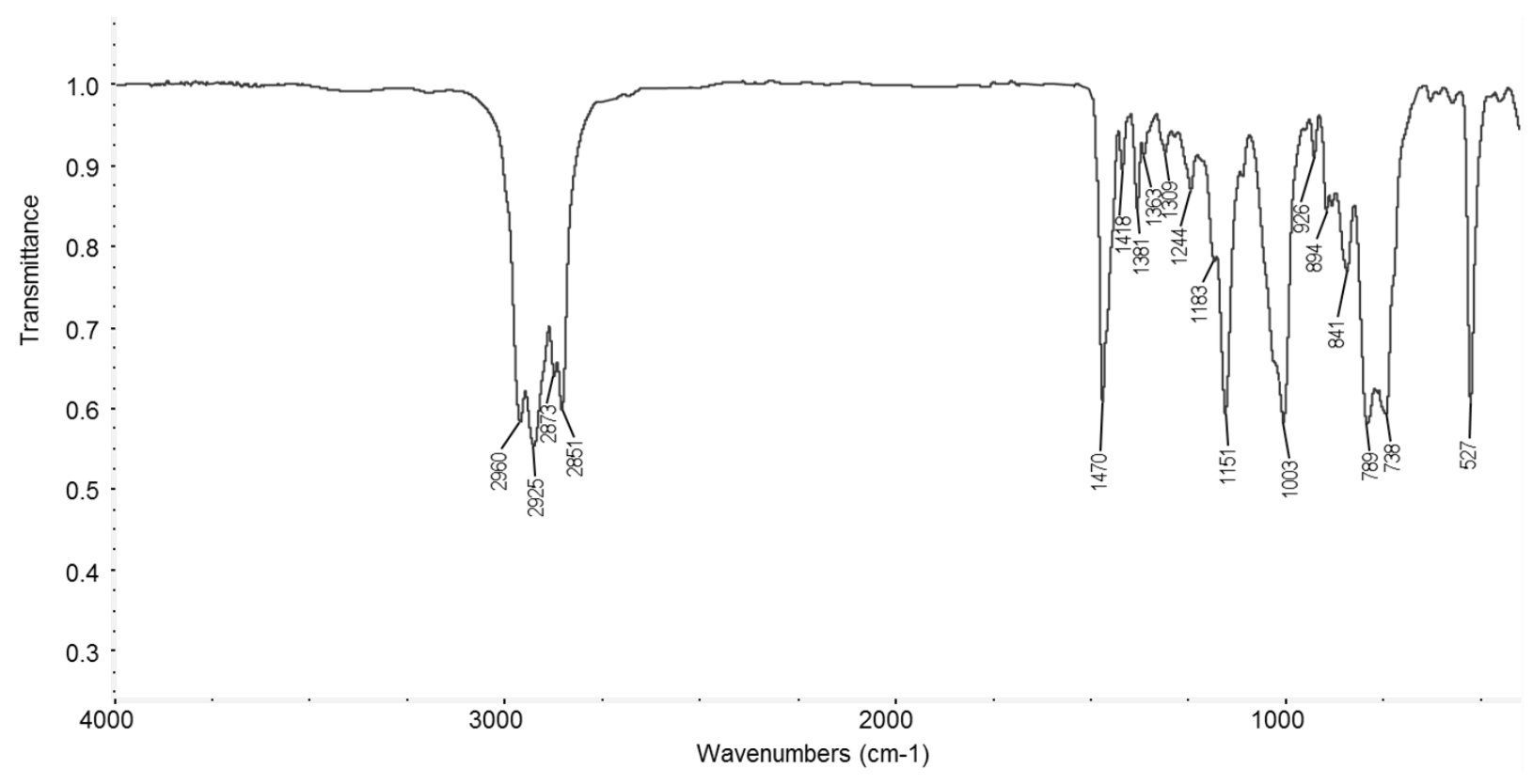

Fig. S32. IR spectra (in $\left.\mathrm{CCl}_{4}\right)$ of $\left(\left(\mathrm{C}_{4} \mathrm{H}_{9}\right)_{4} \mathrm{~N}\right)\left[\mathrm{B}_{10} \mathrm{Cl}_{9} \mathrm{~S}\left(n-\mathrm{C}_{4} \mathrm{H}_{9}\right)_{2}\right]$ 


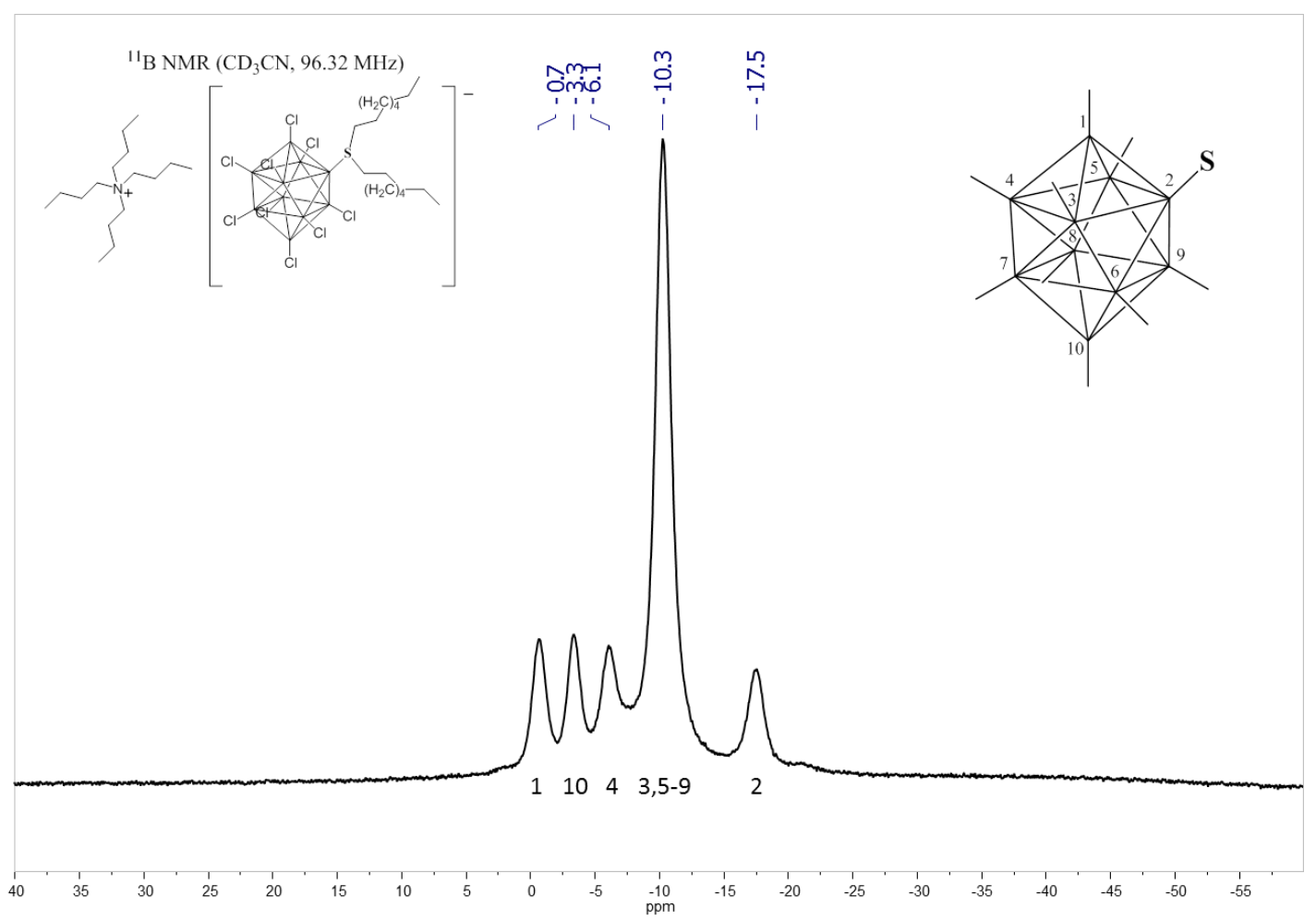

Fig. S33. ${ }^{11}$ B NMR spectrum (acetonitrile-d3, 96.32 MHz) of $\left(\left(\mathrm{C}_{4} \mathrm{H}_{9}\right)_{4} \mathrm{~N}\right)\left[\mathrm{B}_{10} \mathrm{Cl}_{9} \mathrm{~S}\left(n-\mathrm{C}_{8} \mathrm{H}_{17}\right)_{2}\right]$

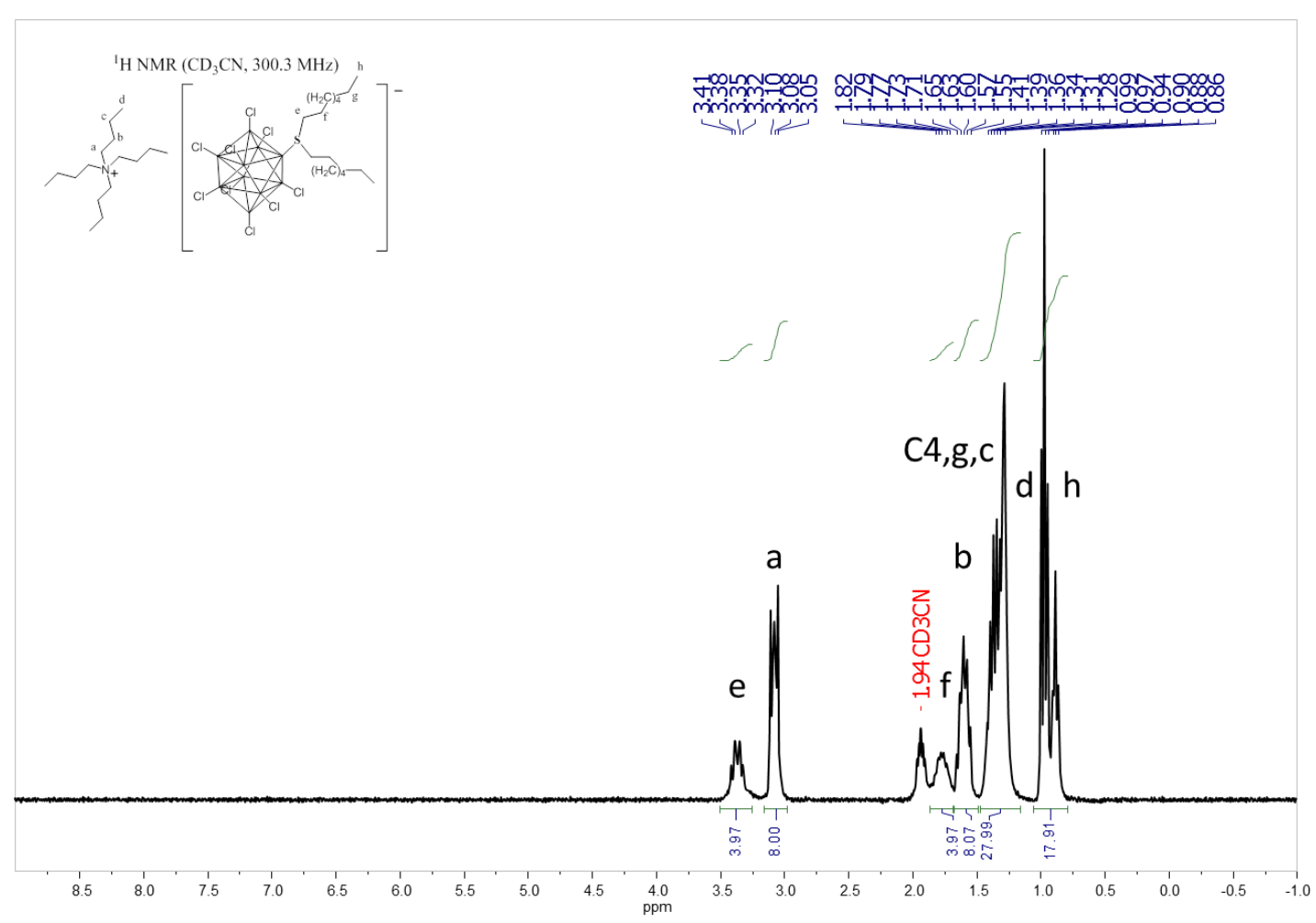

Fig. S34. ${ }^{1} \mathrm{H}$ NMR spectrum (acetonitrile-d3, 300.3 MHz) of $\left(\left(\mathrm{C}_{4} \mathrm{H}_{9}\right)_{4} \mathrm{~N}\right)\left[\mathrm{B}_{10} \mathrm{Cl}_{9} \mathrm{~S}\left(n-\mathrm{C}_{8} \mathrm{H}_{17}\right)_{2}\right]$ 


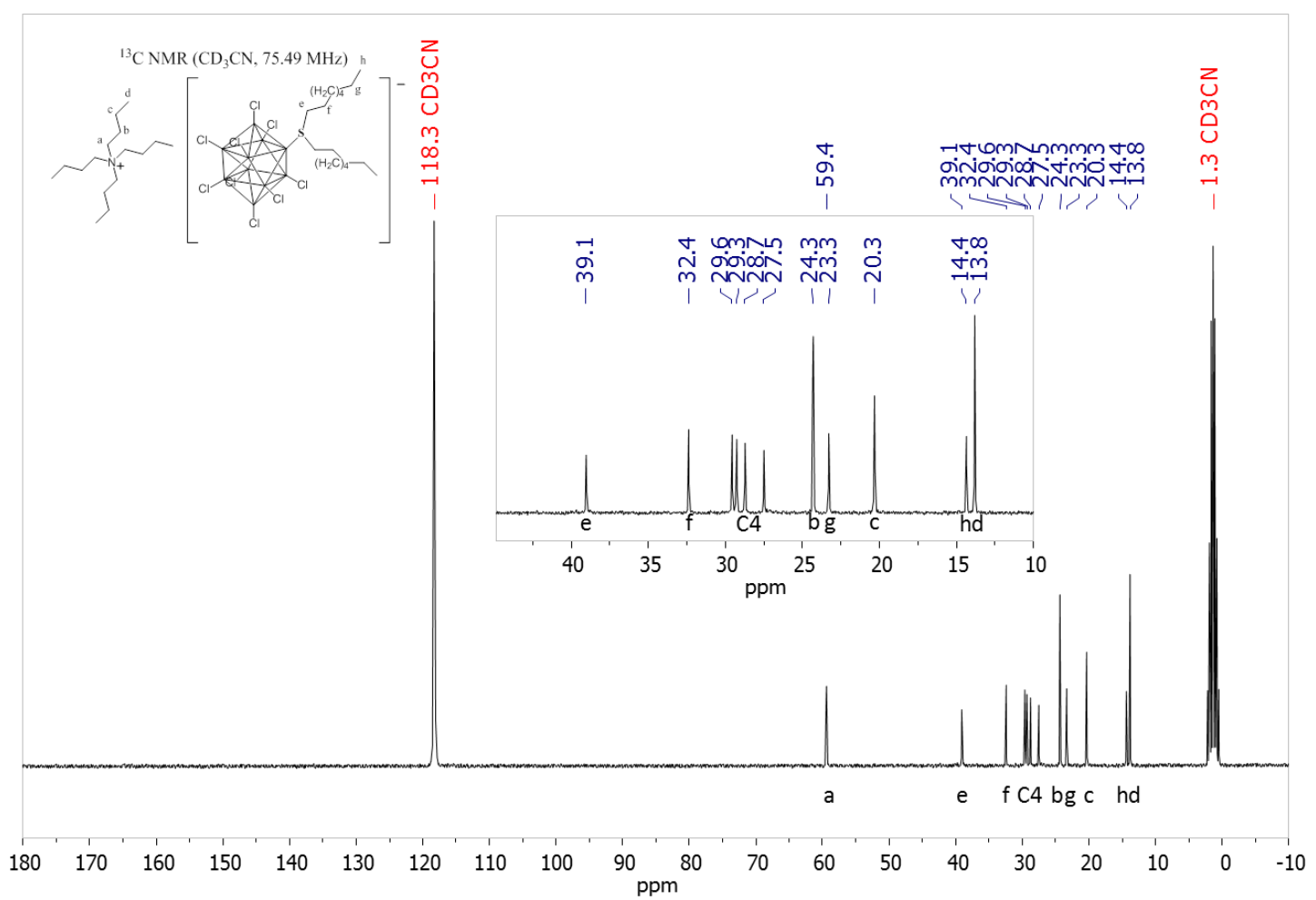

Fig. S35. ${ }^{13} \mathrm{C}$ NMR spectrum (acetonitrile-d3, 75.49 MHz) of $\left(\left(\mathrm{C}_{4} \mathrm{H}_{9}\right)_{4} \mathrm{~N}\right)\left[\mathrm{B}_{10} \mathrm{Cl}_{9} \mathrm{~S}\left(n-\mathrm{C}_{8} \mathrm{H}_{17}\right)_{2}\right]$

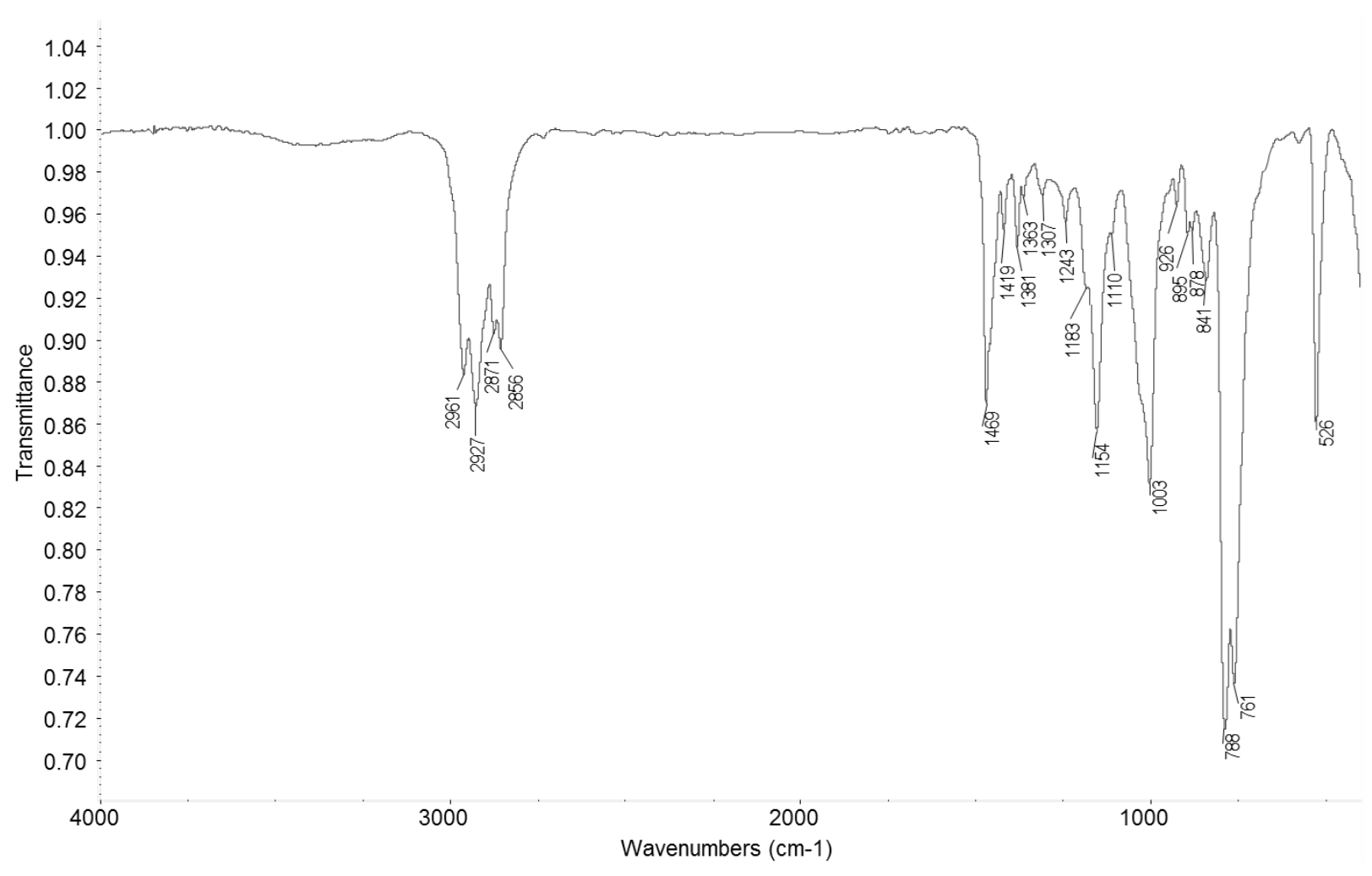

Fig. S36. IR spectra (in $\left.\mathrm{CCl}_{4}\right)$ of $\left(\left(\mathrm{C}_{4} \mathrm{H}_{9}\right)_{4} \mathrm{~N}\right)\left[\mathrm{B}_{10} \mathrm{Cl}{ }_{9} \mathrm{~S}\left(n-\mathrm{C}_{8} \mathrm{H}_{17}\right)_{2}\right]$ 


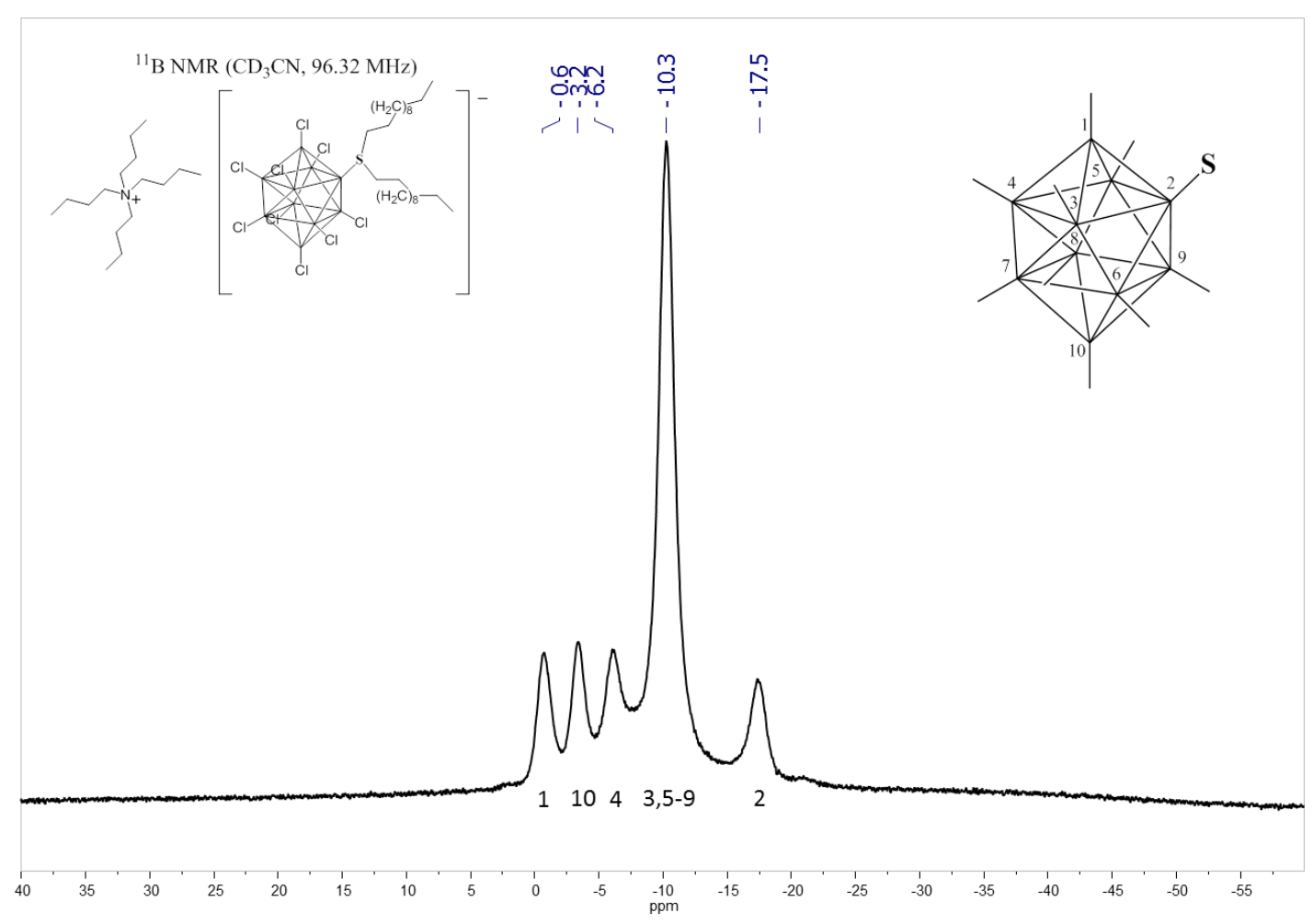

Fig. S37. ${ }^{11} \mathrm{~B}$ NMR spectrum (acetonitrile-d3, 96.32 MHz) of $\left(\left(\mathrm{C}_{4} \mathrm{H}_{9}\right)_{4} \mathrm{~N}\right)\left[\mathrm{B}_{10} \mathrm{Cl}{ }_{9} \mathrm{~S}\left(n-\mathrm{C}_{12} \mathrm{H}_{25}\right)_{2}\right]$

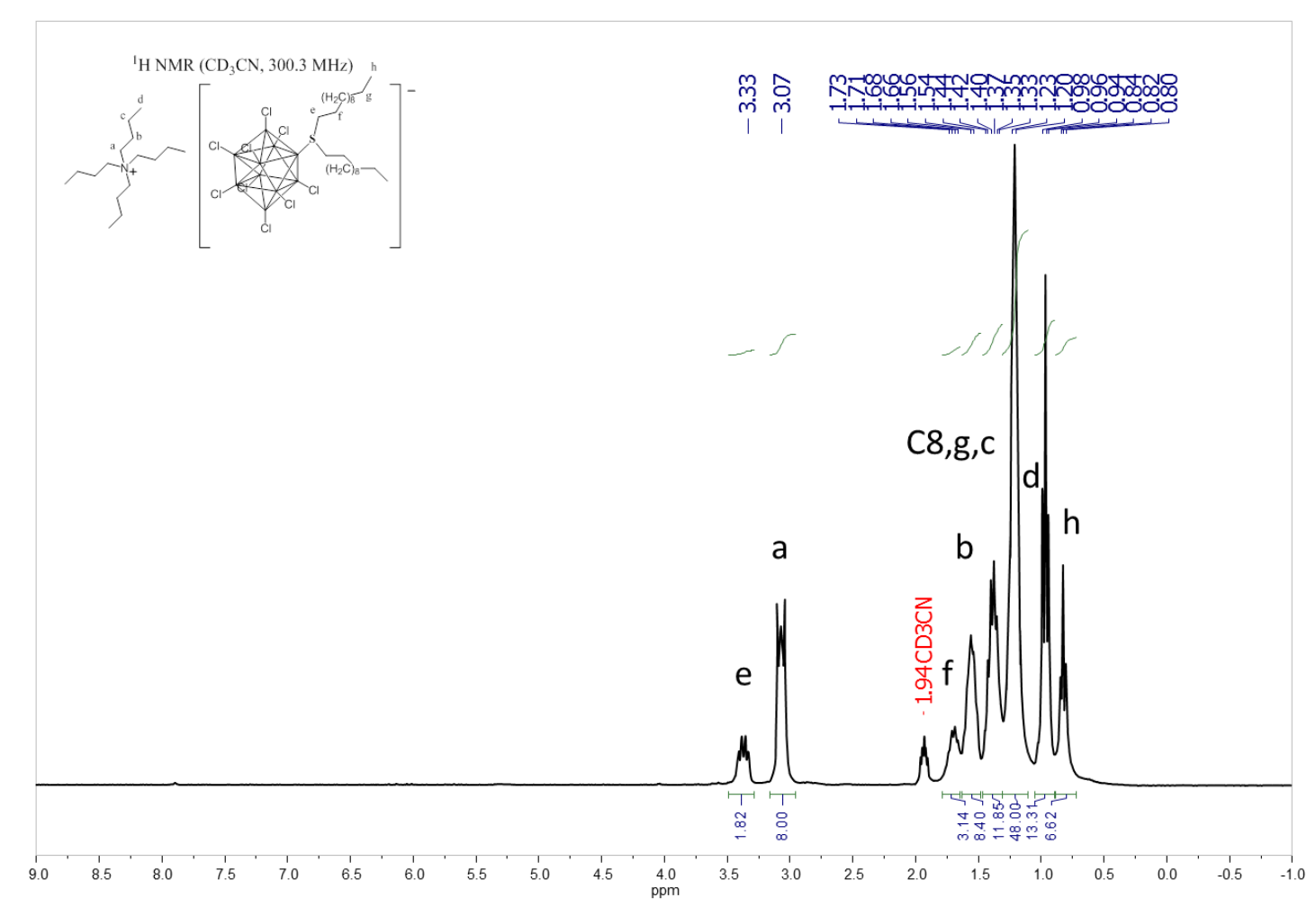

Fig. S38. ${ }^{1} \mathrm{H}$ NMR spectrum (acetonitrile-d3, 300.3 MHz) of $\left(\left(\mathrm{C}_{4} \mathrm{H}_{9}\right)_{4} \mathrm{~N}\right)\left[\mathrm{B}_{10} \mathrm{Cl}_{9} \mathrm{~S}\left(n-\mathrm{C}_{12} \mathrm{H}_{25}\right)_{2}\right]$ 


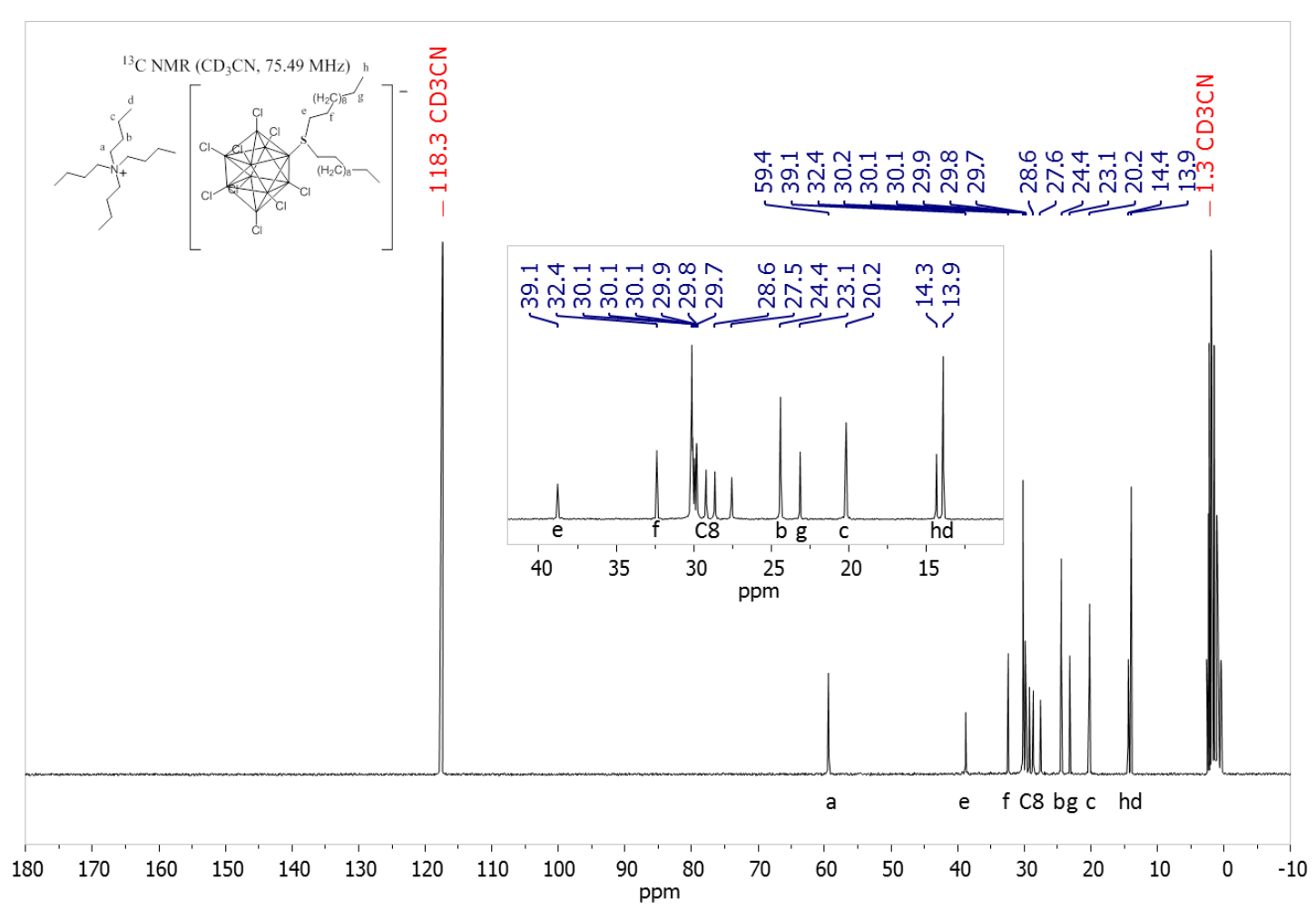

Fig. S39. ${ }^{13} \mathrm{C}$ NMR spectrum (acetonitrile-d3, 75.49 MHz) of $\left(\left(\mathrm{C}_{4} \mathrm{H}_{9}\right)_{4} \mathrm{~N}\right)\left[\mathrm{B}_{10} \mathrm{Cl}_{9} \mathrm{~S}\left(n-\mathrm{C}_{12} \mathrm{H}_{25}\right)_{2}\right]$

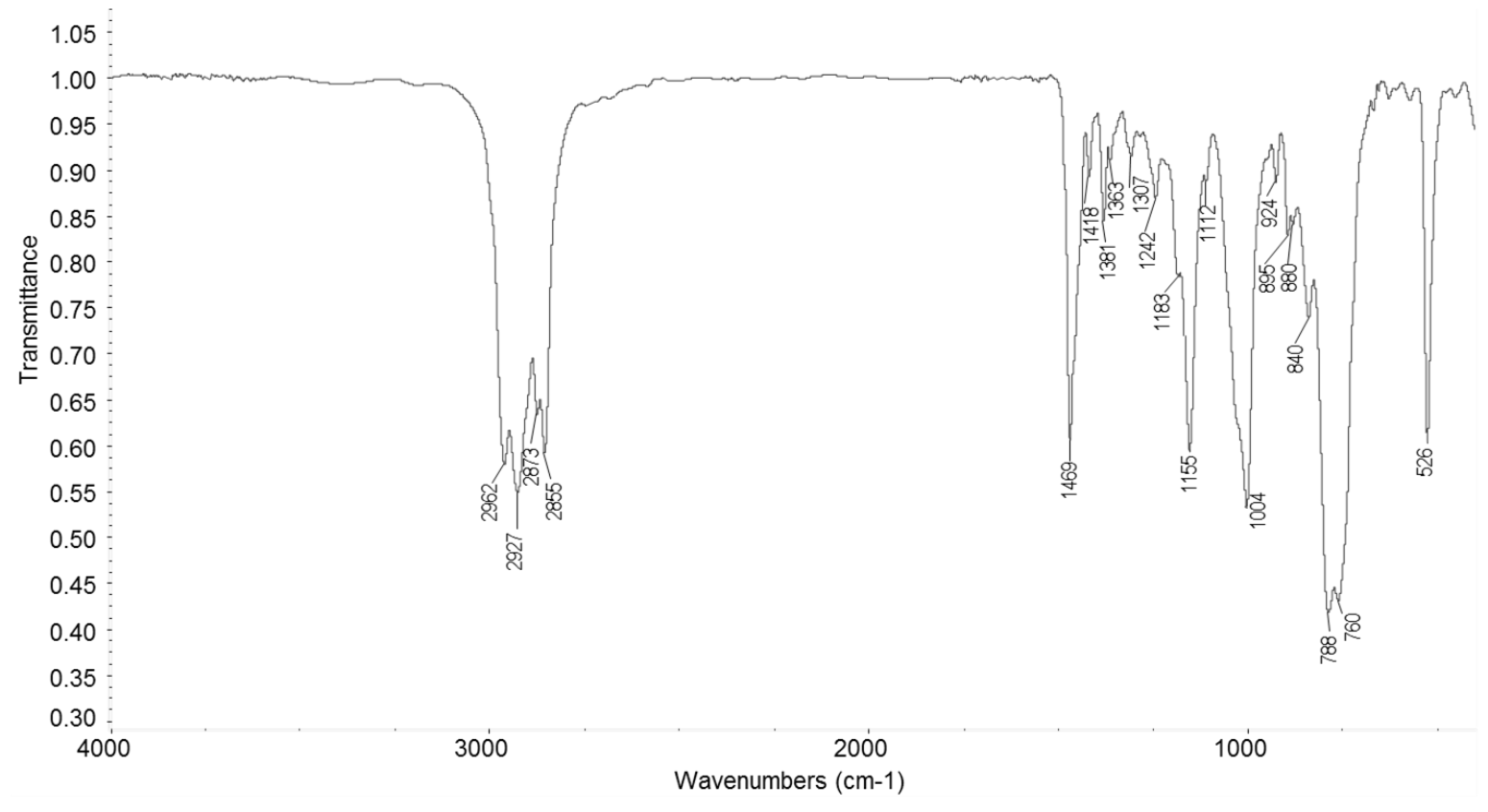

Fig. S40. IR spectra (in $\left.\mathrm{CCl}_{4}\right)$ of $\left(\left(\mathrm{C}_{4} \mathrm{H}_{9}\right)_{4} \mathrm{~N}\right)\left[\mathrm{B}_{10} \mathrm{Cl}_{9} \mathrm{~S}\left(n-\mathrm{C}_{12} \mathrm{H}_{25}\right)_{2}\right]$ 


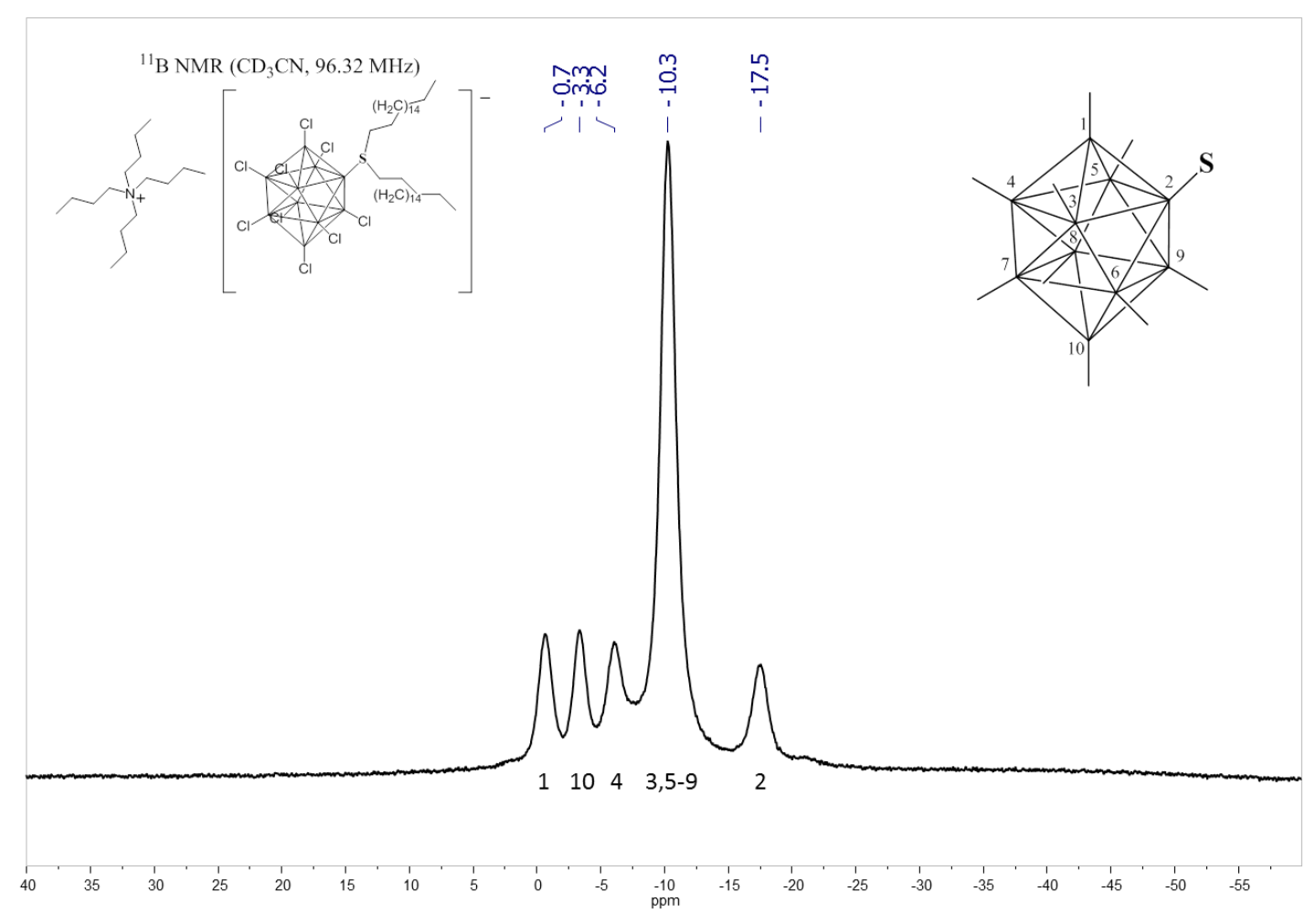

Fig. S41. ${ }^{11} \mathrm{~B}$ NMR spectrum (acetonitrile-d3, 96.32 MHz) of $\left(\left(\mathrm{C}_{4} \mathrm{H}_{9}\right)_{4} \mathrm{~N}\right)\left[\mathrm{B}_{10} \mathrm{Cl}_{9} \mathrm{~S}\left(n-\mathrm{C}_{18} \mathrm{H}_{37}\right)_{2}\right]$

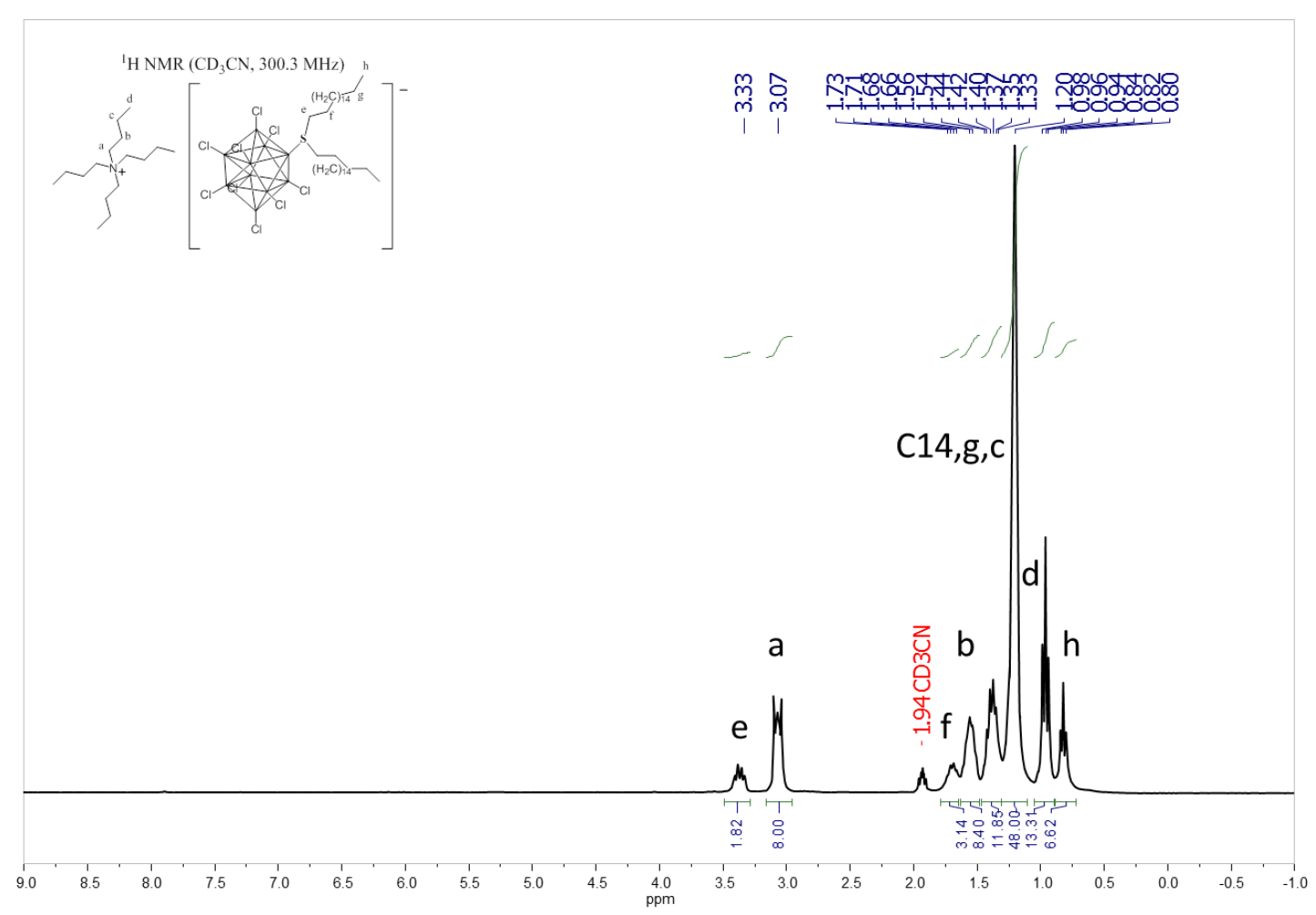

Fig. S42. ${ }^{1} \mathrm{H}$ NMR spectrum (acetonitrile-d3, 300.3 MHz) of $\left(\left(\mathrm{C}_{4} \mathrm{H}_{9}\right)_{4} \mathrm{~N}\right)\left[\mathrm{B}_{10} \mathrm{Cl}_{9} \mathrm{~S}\left(n-\mathrm{C}_{18} \mathrm{H}_{37}\right)_{2}\right]$ 


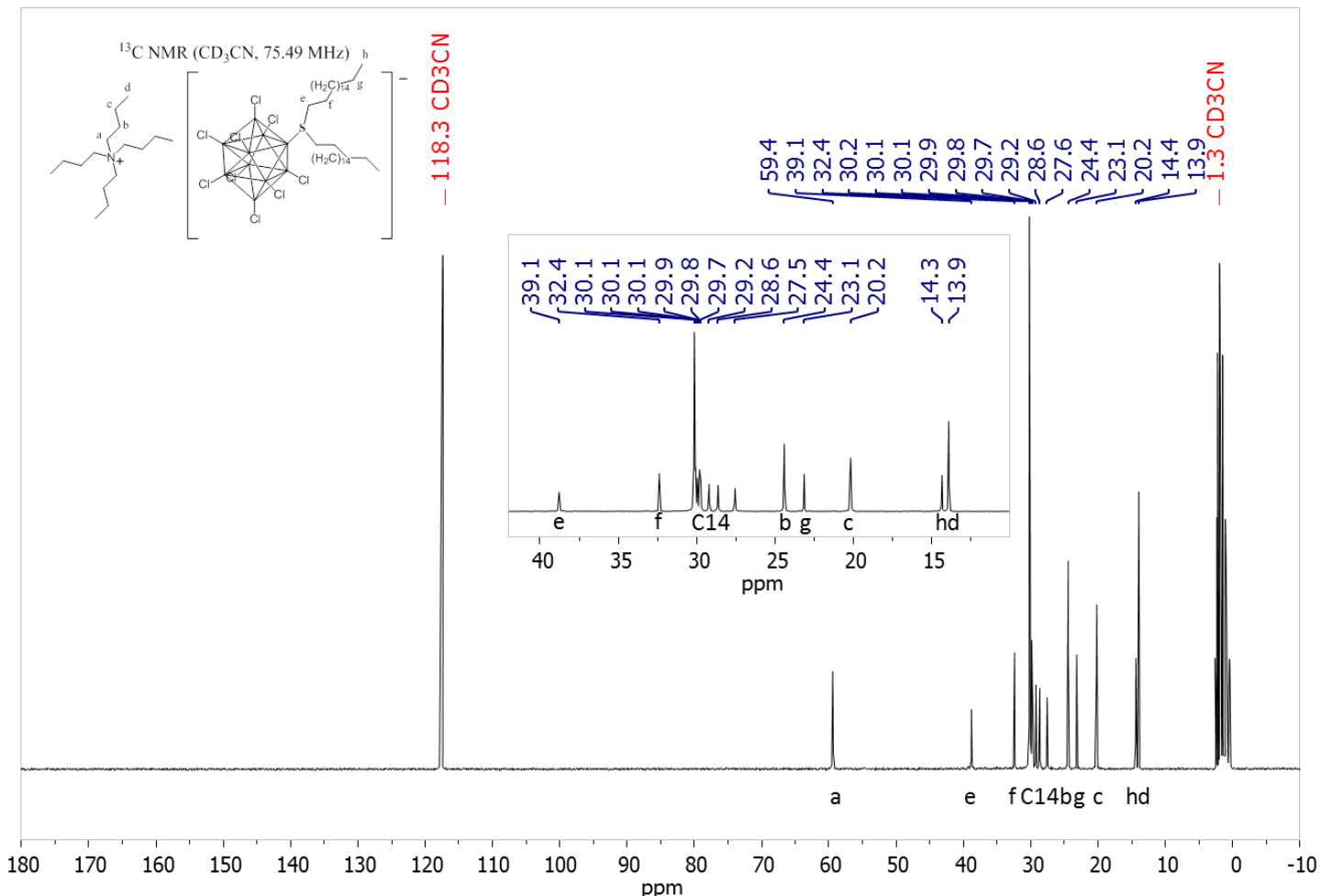

Fig. S43. ${ }^{13} \mathrm{C}$ NMR spectrum (acetonitrile-d3, 75.49 MHz) of $\left(\left(\mathrm{C}_{4} \mathrm{H}_{9}\right)_{4} \mathrm{~N}\right)\left[\mathrm{B}_{10} \mathrm{Cl}_{9} \mathrm{~S}\left(n-\mathrm{C}_{18} \mathrm{H}_{37}\right)_{2}\right]$

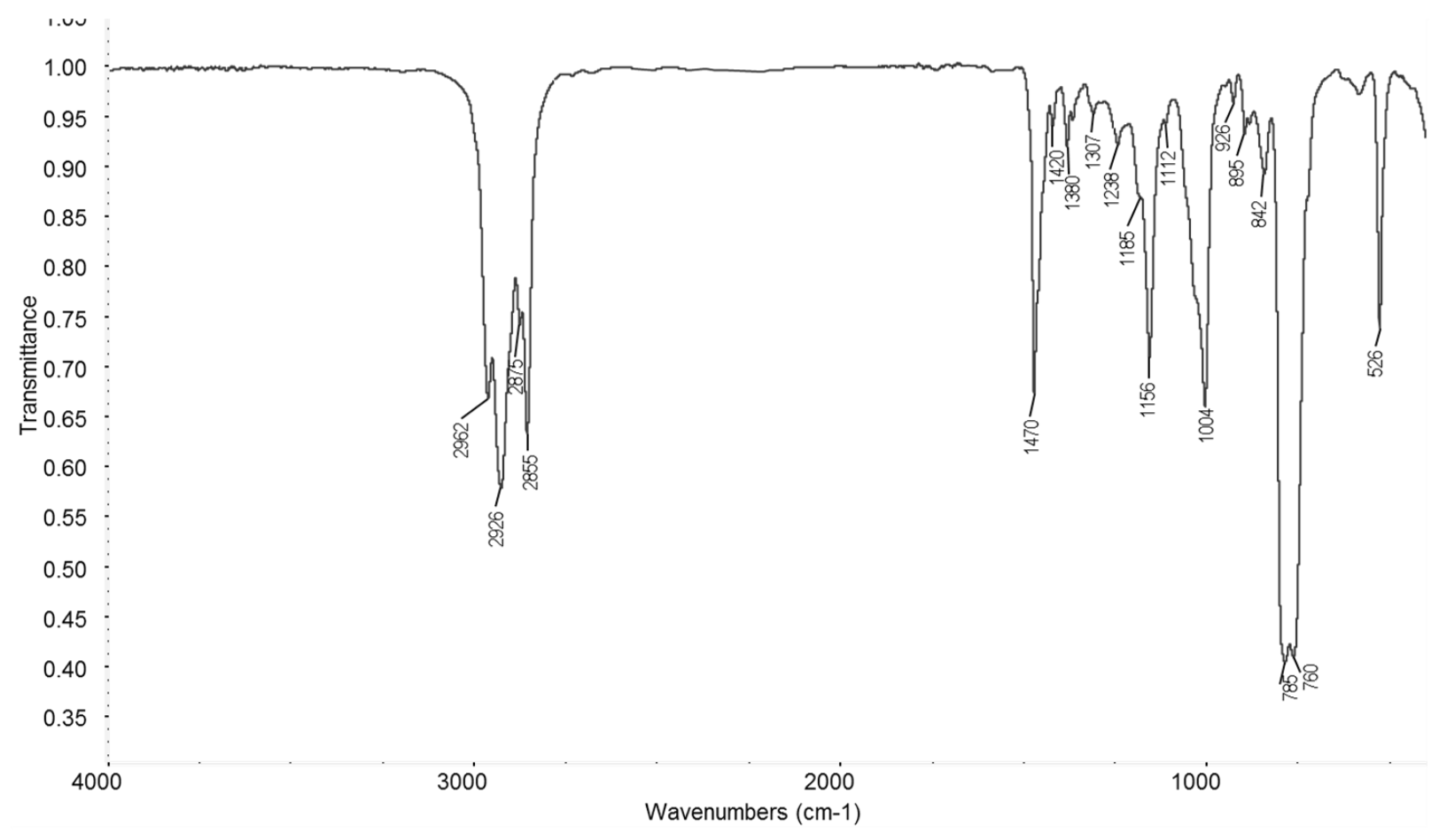

Fig. S44. IR spectra (in $\left.\mathrm{CCl}_{4}\right)$ of $\left(\left(\mathrm{C}_{4} \mathrm{H}_{9}\right)_{4} \mathrm{~N}\right)\left[\mathrm{B}_{10} \mathrm{Cl}_{9} \mathrm{~S}\left(n-\mathrm{C}_{18} \mathrm{H}_{37}\right)_{2}\right]$ 


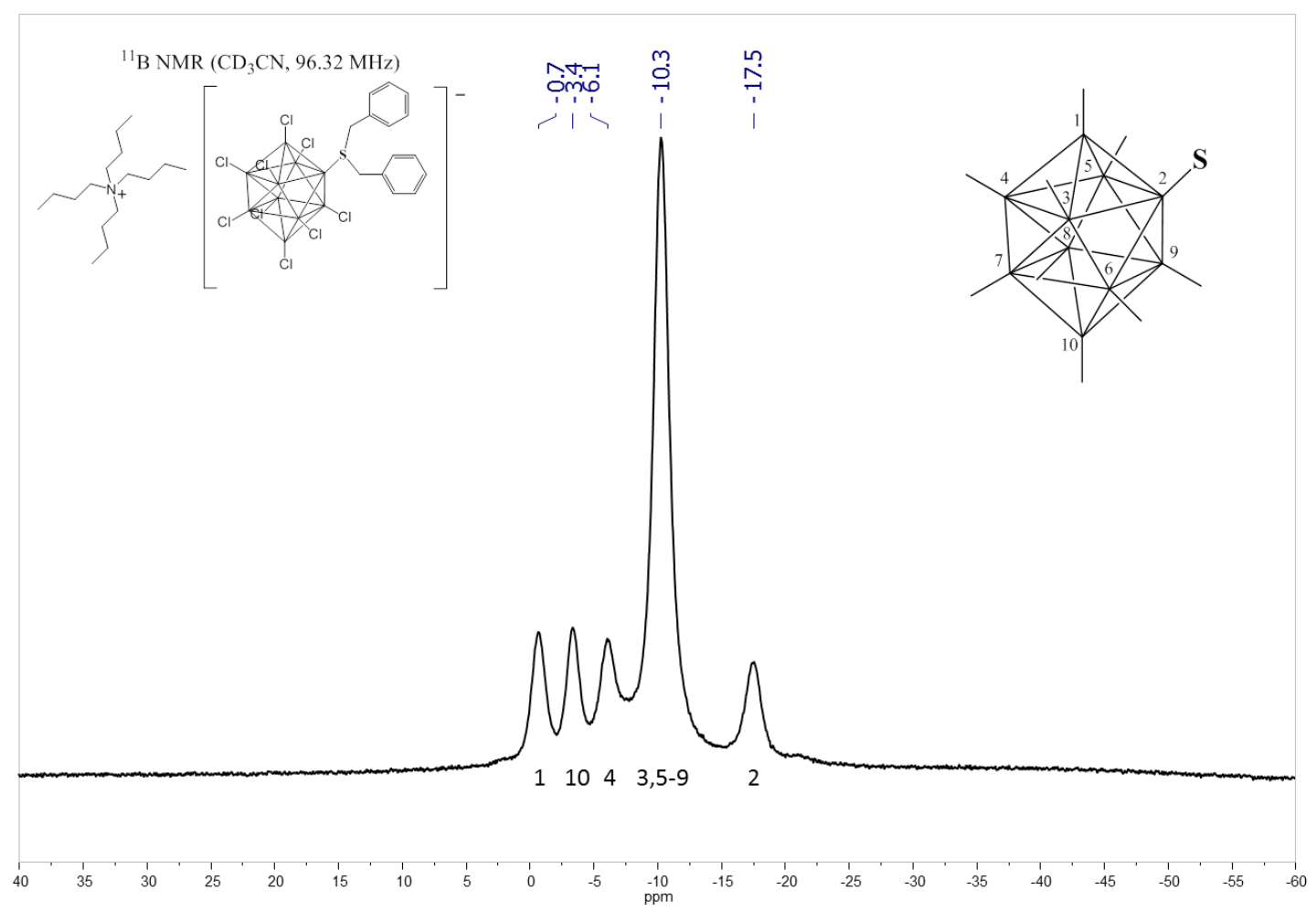

Fig. S45. ${ }^{11}$ B NMR spectrum (acetonitrile-d3, 96.32 MHz) of $\left(\left(\mathrm{C}_{4} \mathrm{H}_{9}\right)_{4} \mathrm{~N}\right)\left[\mathrm{B}_{10} \mathrm{Cl}_{9} \mathrm{~S}\left(\mathrm{CH}_{2} \mathrm{Ph}\right)_{2}\right]$

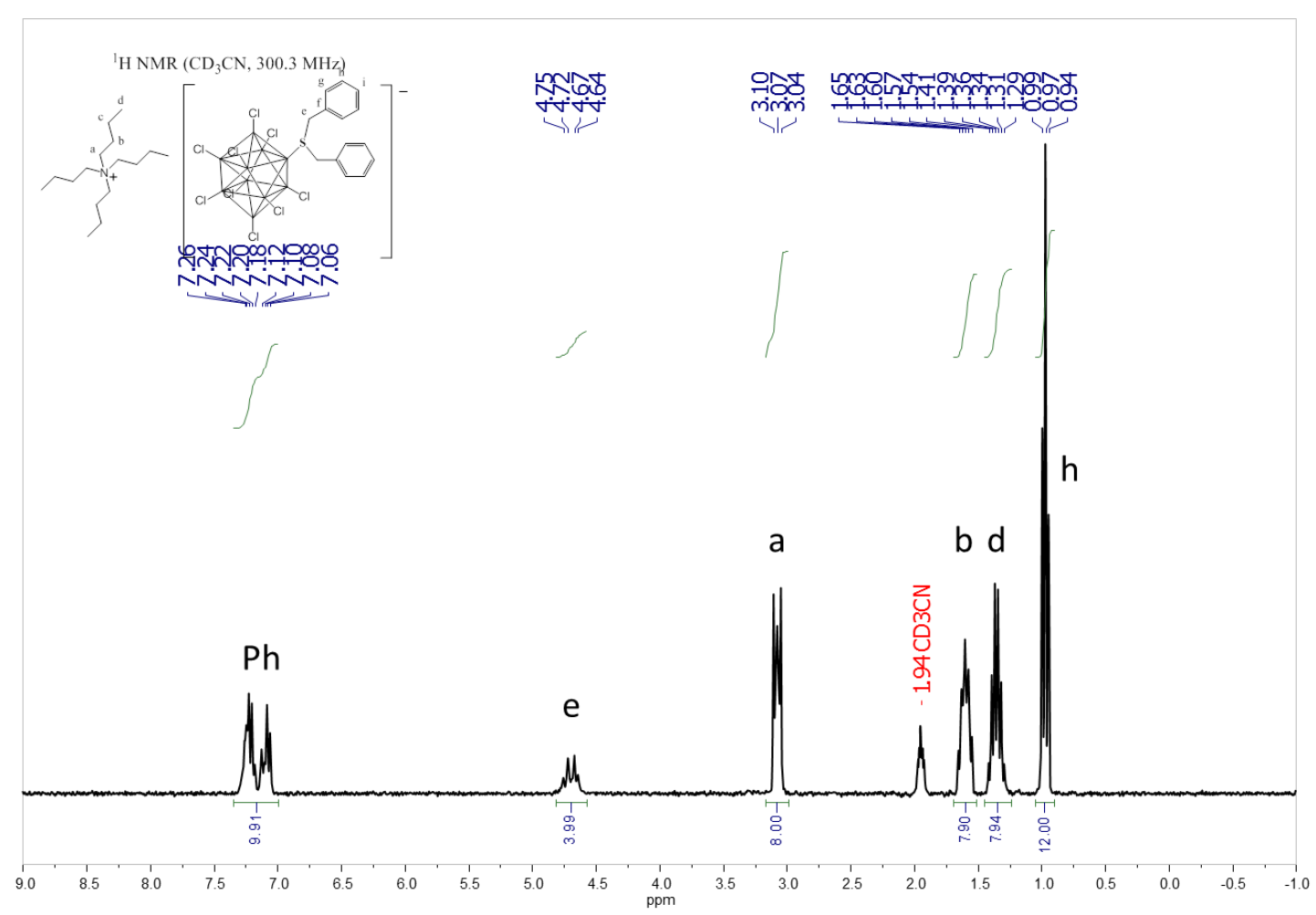

Fig. S46. ${ }^{1} \mathrm{H}$ NMR spectrum (acetonitrile-d3, 300.3 MHz) of $\left(\left(\mathrm{C}_{4} \mathrm{H}_{9}\right)_{4} \mathrm{~N}\right)\left[\mathrm{B}_{10} \mathrm{Cl}_{9} \mathrm{~S}\left(\mathrm{CH}_{2} \mathrm{Ph}\right)_{2}\right]$ 


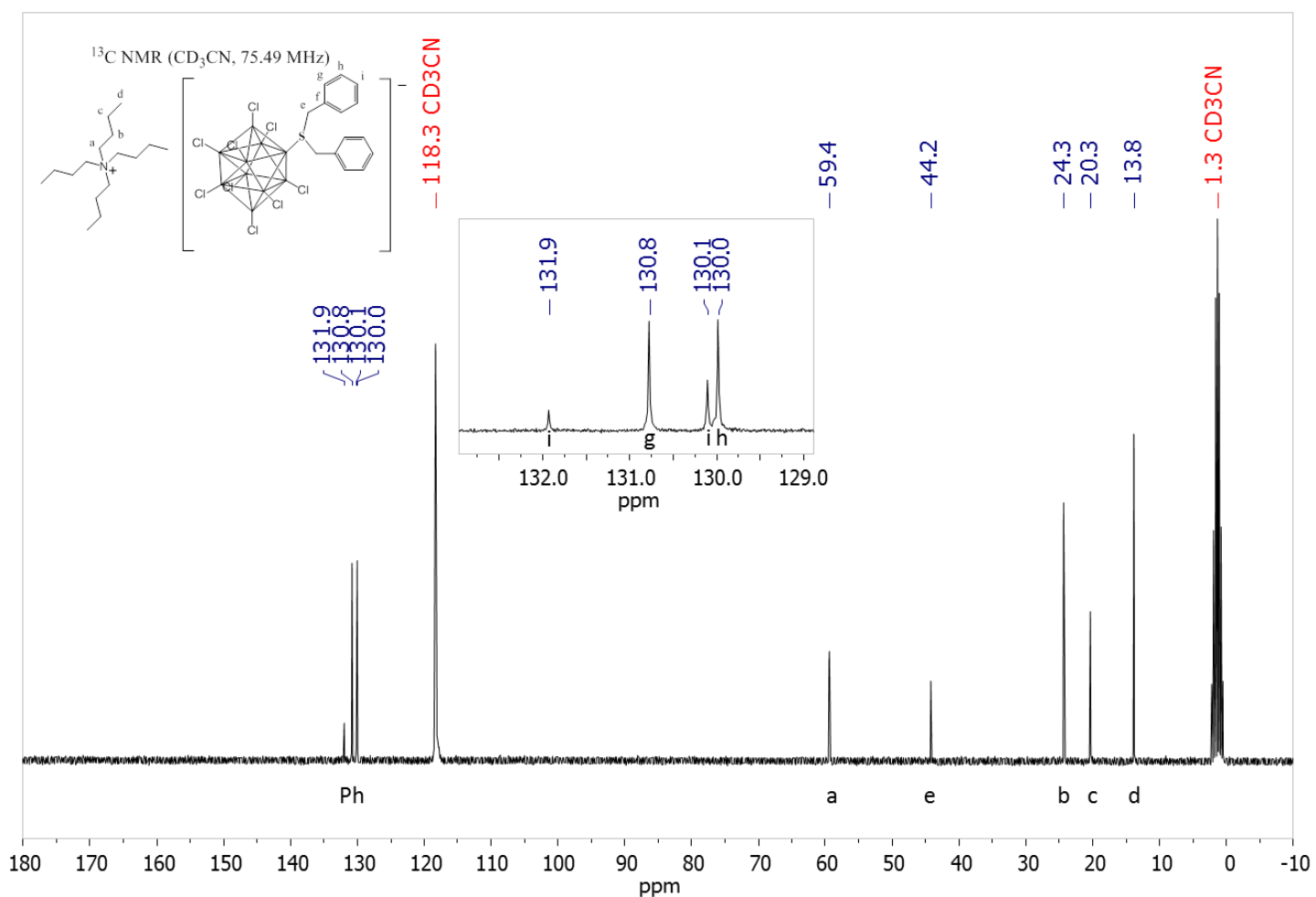

Fig. S47. ${ }^{13} \mathrm{C}$ NMR spectrum (acetonitrile-d3, 75.49 MHz) of $\left(\left(\mathrm{C}_{4} \mathrm{H}_{9}\right)_{4} \mathrm{~N}\right)\left[\mathrm{B}_{10} \mathrm{Cl}_{9} \mathrm{~S}\left(\mathrm{CH}_{2} \mathrm{Ph}\right)_{2}\right]$

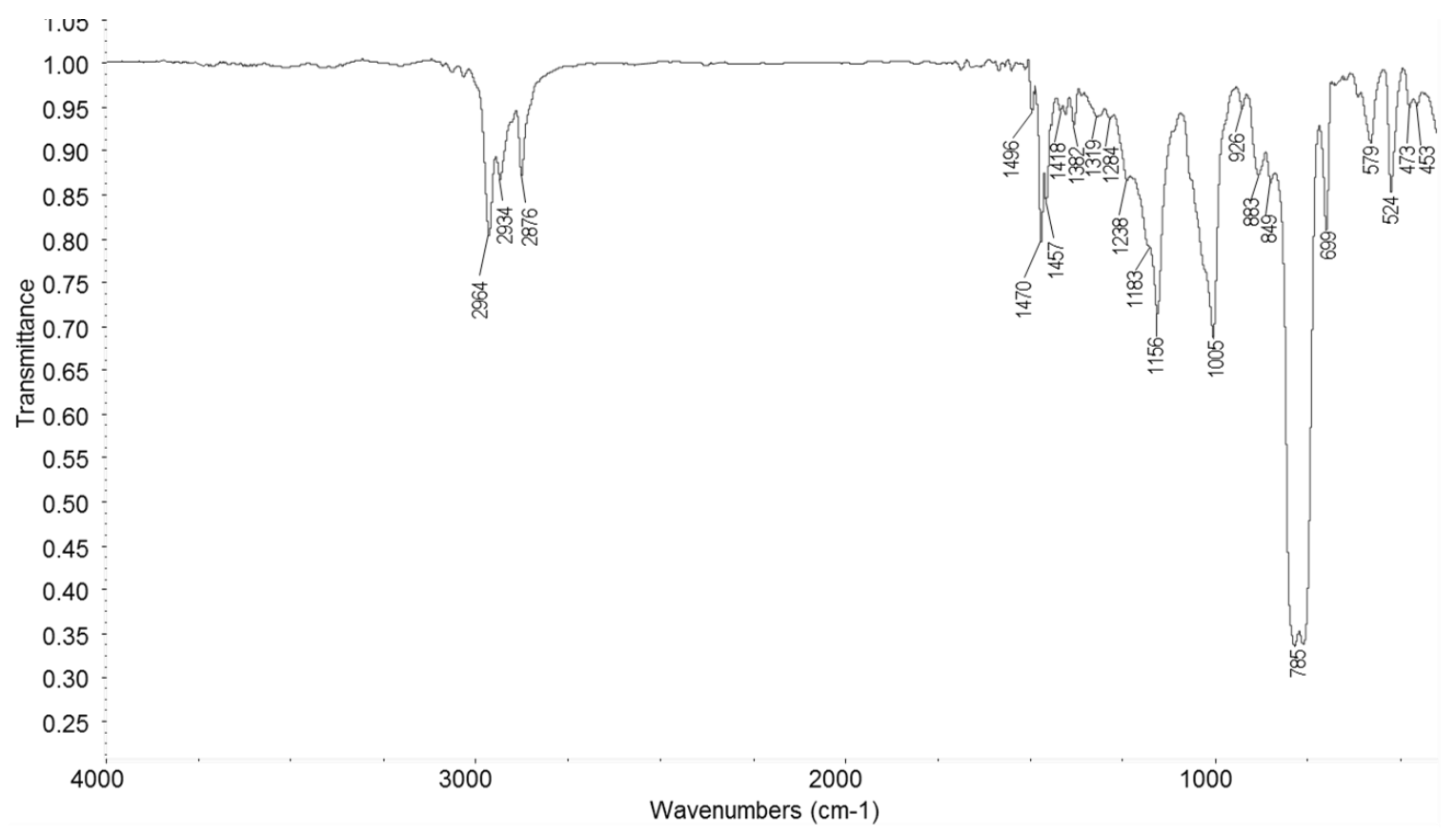

Fig. S48. IR spectra (in $\left.\mathrm{CCl}_{4}\right)$ of $\left(\left(\mathrm{C}_{4} \mathrm{H}_{9}\right)_{4} \mathrm{~N}\right)\left[\mathrm{B}_{10} \mathrm{Cl}_{9} \mathrm{~S}\left(\mathrm{CH}_{2} \mathrm{Ph}\right)_{2}\right]$ 


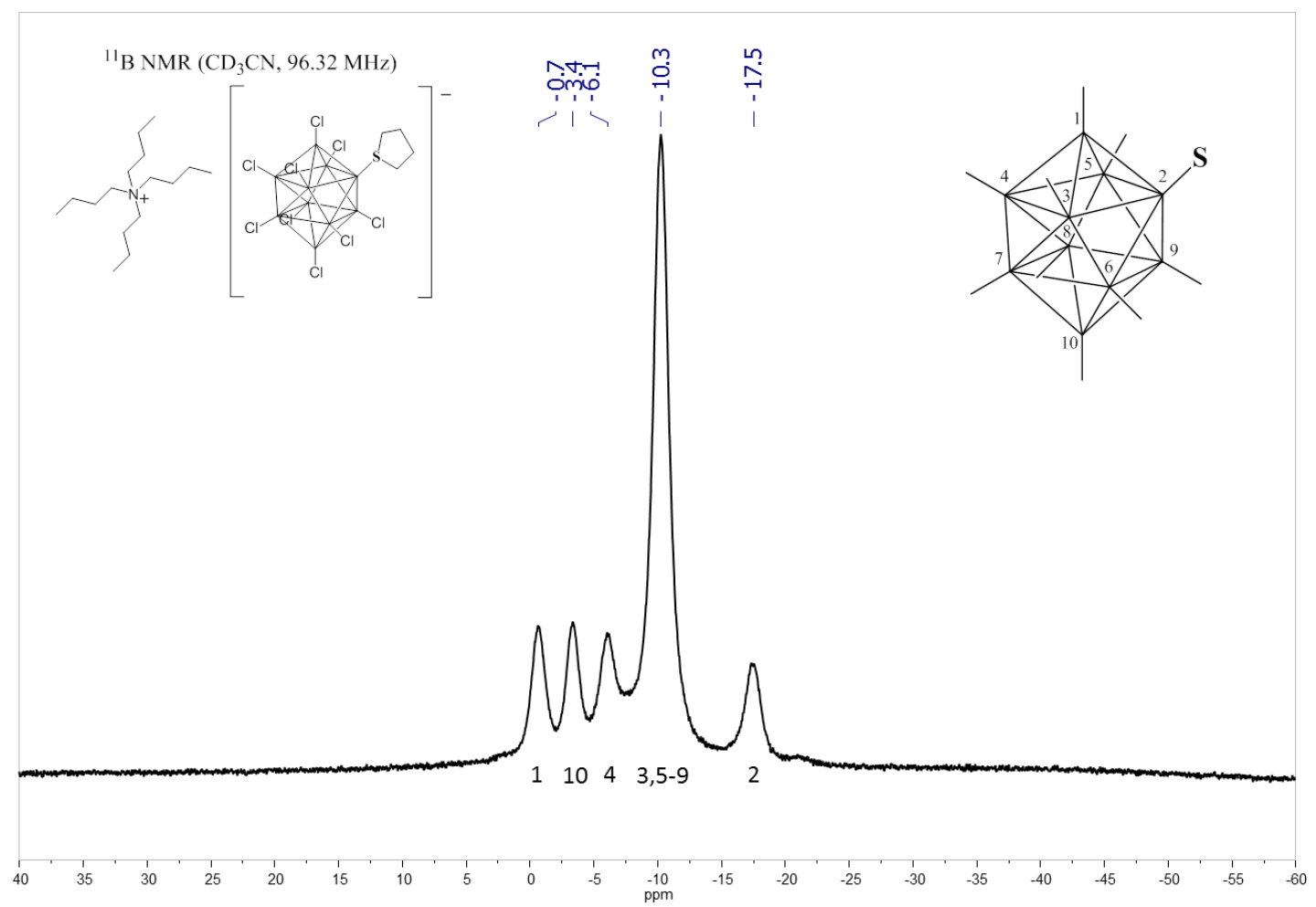

Fig. S49. ${ }^{11} \mathrm{~B}$ NMR spectrum (acetonitrile-d3, 96.32 MHz) of $\left(\left(\mathrm{C}_{4} \mathrm{H}_{9}\right)_{4} \mathrm{~N}\right)\left[\mathrm{B}_{10} \mathrm{Cl}_{9} \mathrm{~S}\right.$-cyclo-S $\left.\left(\mathrm{CH}_{2}\right)_{4}\right]$

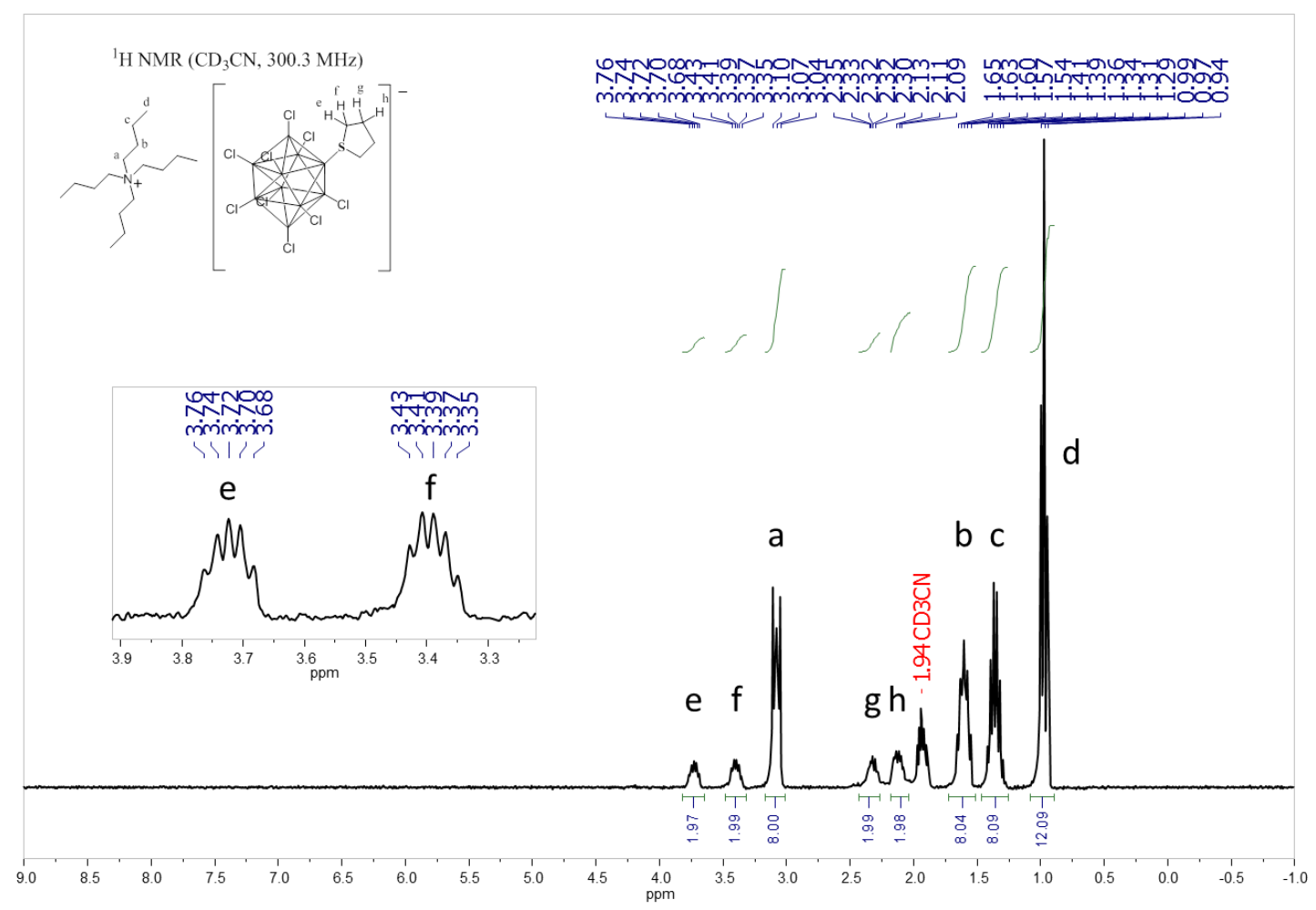

Fig. S50. ${ }^{1} \mathrm{H}$ NMR spectrum (acetonitrile-d $3,300.3 \mathrm{MHz}$ ) of $\left(\left(\mathrm{C}_{4} \mathrm{H}_{9}\right)_{4} \mathrm{~N}\right)\left[\mathrm{B}_{10} \mathrm{Cl}_{9} \mathrm{~S}\right.$-cyclo-S $\left.\left(\mathrm{CH}_{2}\right)_{4}\right]$ 


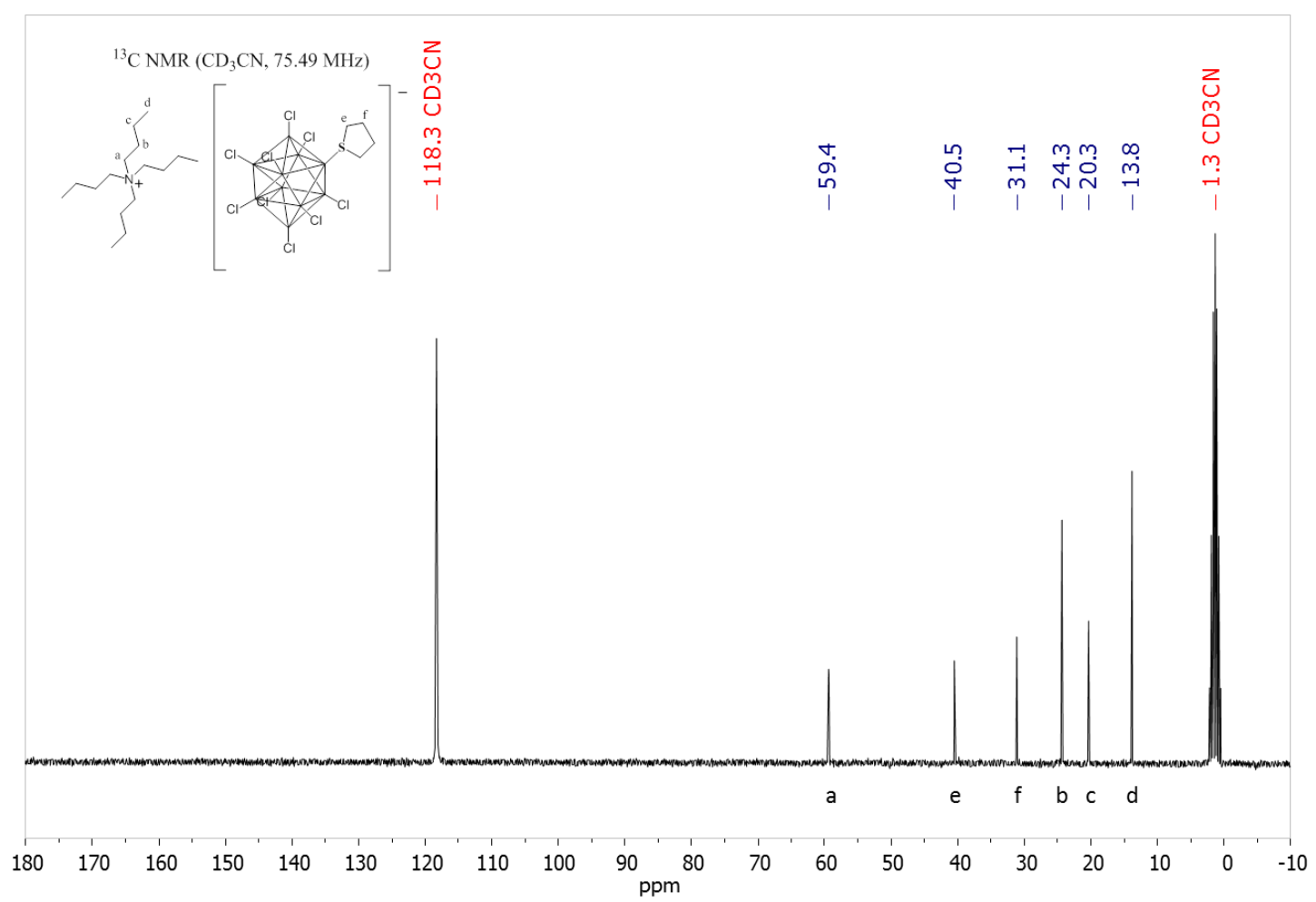

Fig. S51. ${ }^{13} \mathrm{C}$ NMR spectrum (acetonitrile-d3, 75.49 MHz) of $\left(\left(\mathrm{C}_{4} \mathrm{H}_{9}\right)_{4} \mathrm{~N}\right)\left[\mathrm{B}_{10} \mathrm{Cl}_{9}\right.$-cyclo-S $\left.\left(\mathrm{CH}_{2}\right)_{4}\right]$

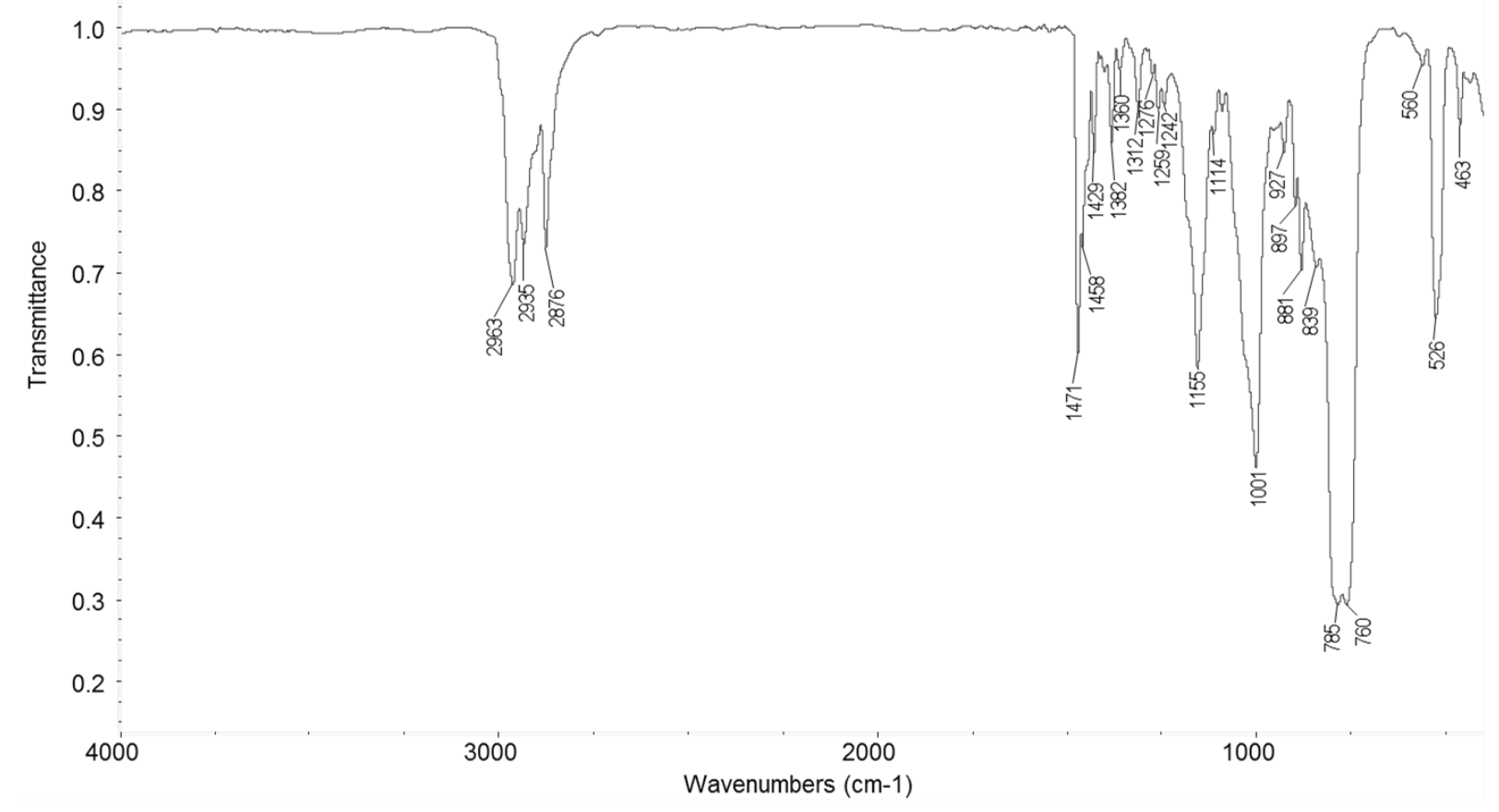

Fig. S52. IR spectra (in $\left.\mathrm{CCl}_{4}\right)$ of $\left(\left(\mathrm{C}_{4} \mathrm{H}_{9}\right)_{4} \mathrm{~N}\right)\left[\mathrm{B}_{10} \mathrm{Cl}_{9}\right.$-cyclo-S $\left.\left(\mathrm{C}_{4} \mathrm{H}_{8}\right)\right]$ 


\section{ESI-MS}

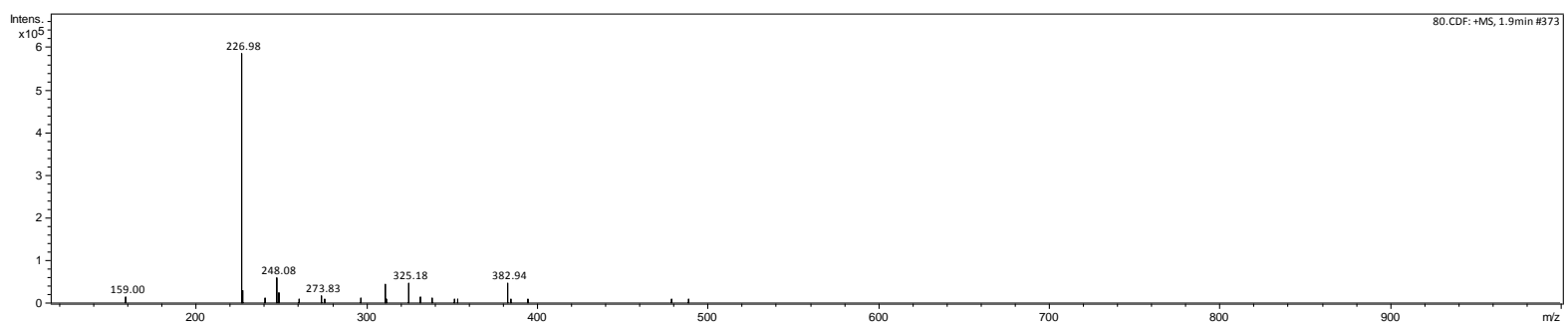

Fig. S53. ESI-MS background spectrum in the negative ionization mode
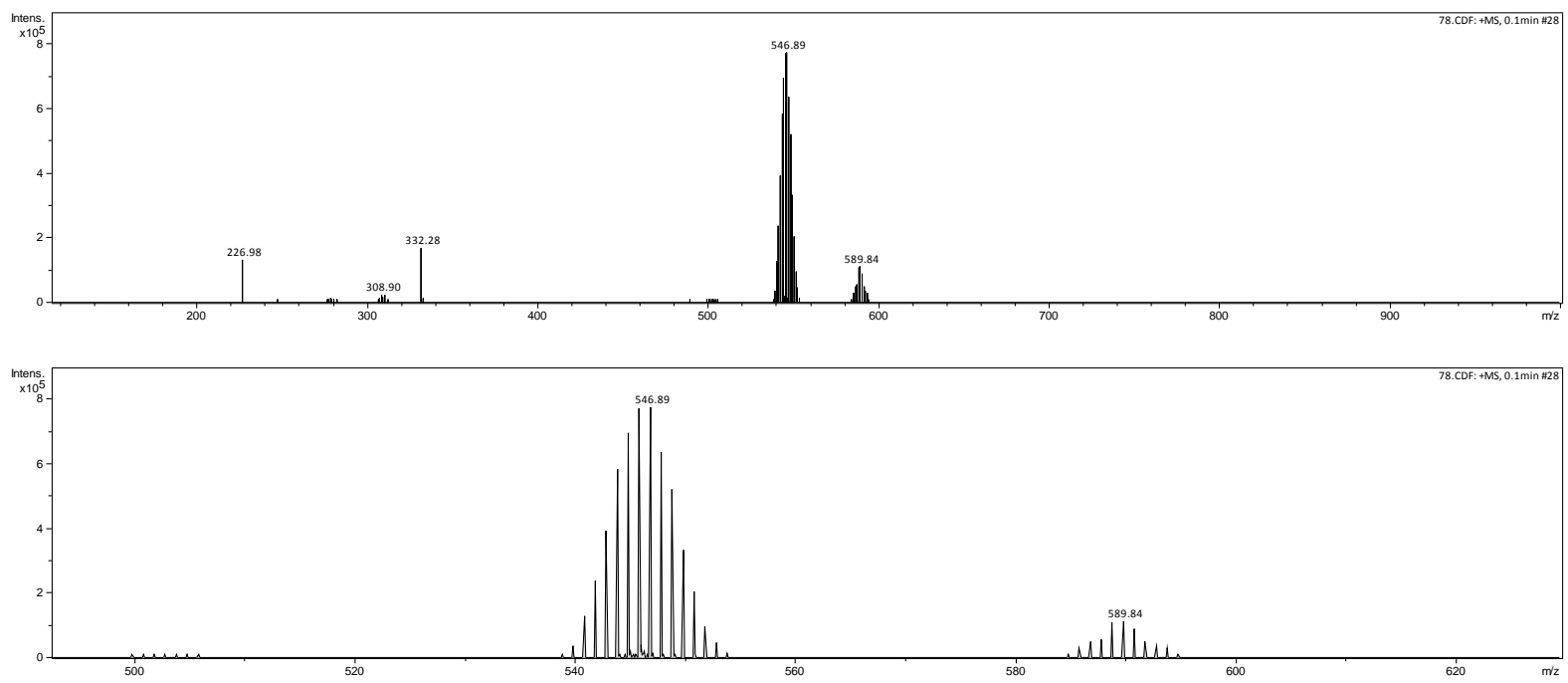

Fig. S54. ESI-MS spectrum of compound $\left.1\left(\left(\mathrm{C}_{4} \mathrm{H}_{9}\right)_{4} \mathrm{~N}\right)\left[\mathrm{B}_{10} \mathrm{Cl} 9 \mathrm{~S}\left(i-\mathrm{C}_{3} \mathrm{H}_{7}\right)_{2}\right]\right)$ in the negative ionization mode

Negative ESI-MS, m/z: found for $\left[\mathrm{B}_{10} \mathrm{Cl}{ }_{9} \mathrm{~S}\left(i-\mathrm{C}_{3} \mathrm{H}_{7}\right)_{2}\right]^{-}=546.89$, calc. for C6H14B10C19S1 = 544.89;

Negative ESI-MS, m/z: found for $\left(\left[\mathrm{B}_{10} \mathrm{Cl}_{9} \mathrm{~S}\left(i-\mathrm{C}_{3} \mathrm{H}_{7}\right)_{2}\right][\mathrm{HCOOH}]\right)^{-}=589.84$, calc. for C7H16B10C19O2S1 = 590.90;

Negative ESI-MS, m/z: found for $\left(\left[\left(\mathrm{C}_{4} \mathrm{H}_{9}\right)_{4} \mathrm{~N}\right][\mathrm{HCOO}]_{2}\right)^{-}=332.28$, calc. for C18H38N1O4 = 332.28.
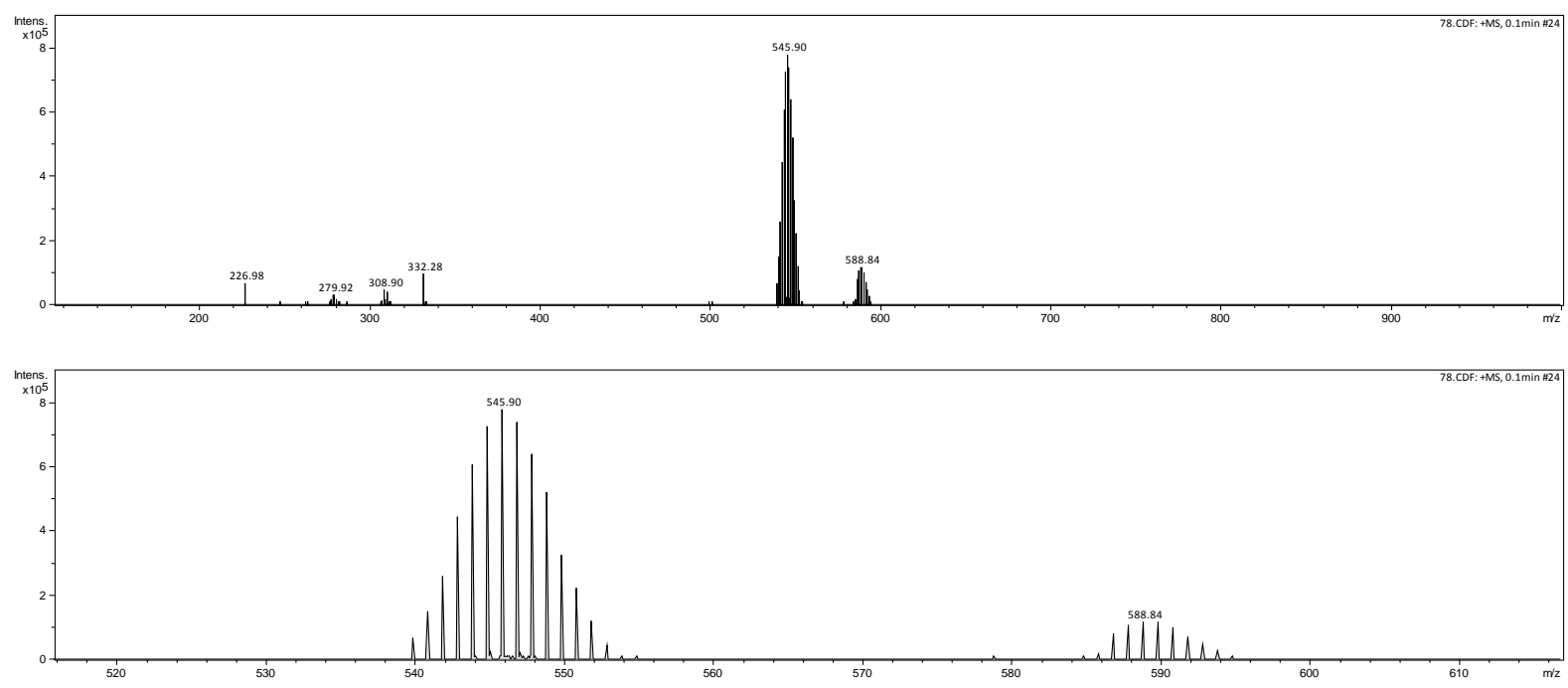
Fig. S55. ESI-MS spectrum of compound $\left.2\left(\left(\mathrm{C}_{4} \mathrm{H}_{9}\right)_{4} \mathrm{~N}\right)\left[\mathrm{B}_{10} \mathrm{Cl}{ }_{9} \mathrm{~S}\left(n-\mathrm{C}_{3} \mathrm{H}_{7}\right)_{2}\right]\right)$ in the negative ionization mode

Negative ESI-MS, m/z: found for $\left(\left[\mathrm{B}_{10} \mathrm{Cl}_{9} \mathrm{~S}\left(i-\mathrm{C}_{3} \mathrm{H}_{7}\right)_{2}\right]\right)^{-}=546.90$, calc. for C6H14B10C19S1 = 544.89;

Negative ESI-MS, m/z: found for $\left(\left[\mathrm{B}_{10} \mathrm{Cl}_{9} \mathrm{~S}\left(i-\mathrm{C}_{3} \mathrm{H}_{7}\right)_{2}\right][\mathrm{HCOOH}]\right)^{-}=588.84$, calc. for $\mathrm{C} 7 \mathrm{H} 16 \mathrm{~B} 10 \mathrm{Cl} 19 \mathrm{O} 2 \mathrm{~S} 1=590.90$;

Negative ESI-MS, m/z: found for $\left(\left[\left(\mathrm{C}_{4} \mathrm{H}_{9}\right)_{4} \mathrm{~N}\right][\mathrm{HCOO}]_{2}\right)^{-}=332.28$, calc. for $\mathrm{C} 18 \mathrm{H} 38 \mathrm{~N} 1 \mathrm{O} 4=332.28$.
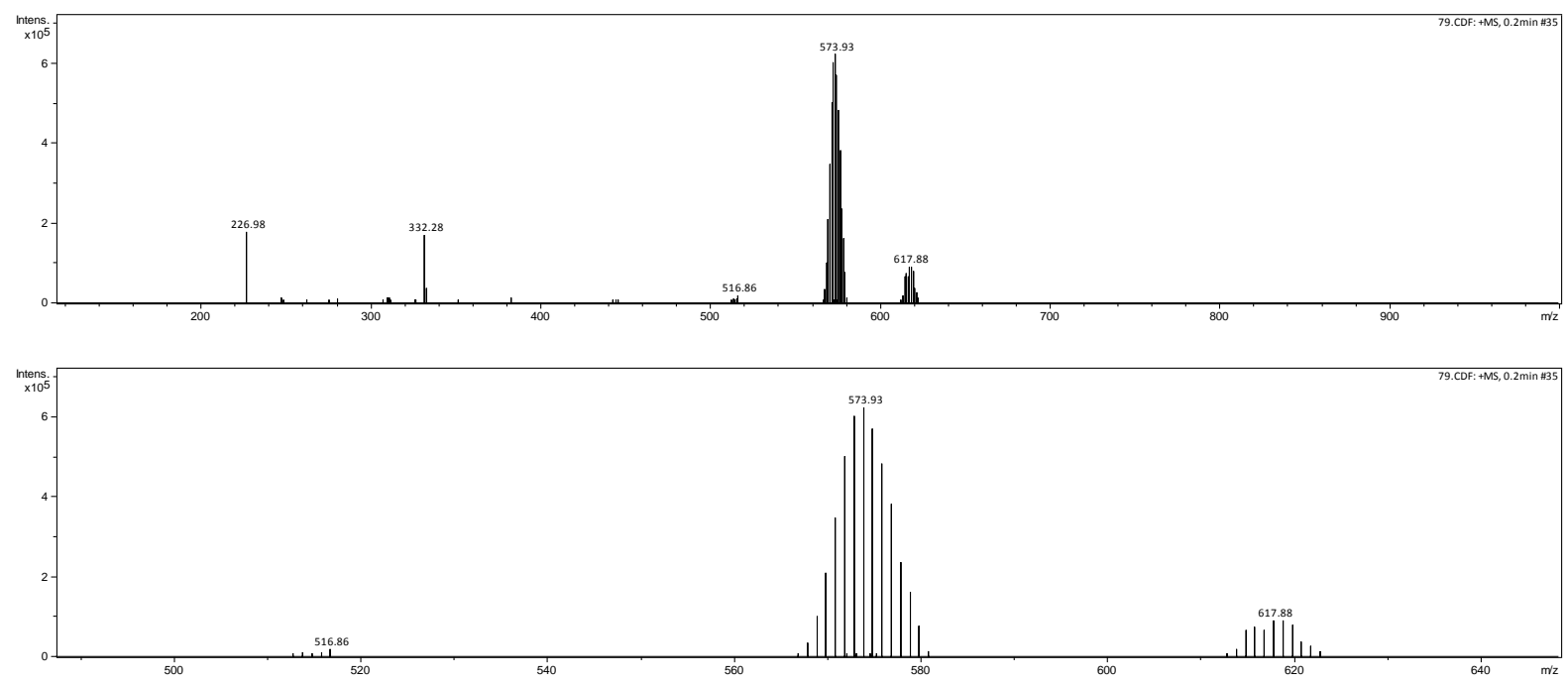

Fig. S56. ESI-MS spectrum of compound $\left.3\left(\left(\mathrm{C}_{4} \mathrm{H}_{9}\right)_{4} \mathrm{~N}\right)\left[\mathrm{B}_{10} \mathrm{Cl}_{9} \mathrm{~S}\left(n-\mathrm{C}_{4} \mathrm{H}_{9}\right)_{2}\right]\right)$ in the negative ionization mode

Negative ESI-MS, m/z: found for $\left(\left[\mathrm{B}_{10} \mathrm{Cl}{ }_{9} \mathrm{~S}\left(n-\mathrm{C}_{4} \mathrm{H}_{9}\right)_{2}\right]\right)^{-}=573.93$, calc. for $\mathrm{C} 8 \mathrm{H} 18 \mathrm{~B} 10 \mathrm{Cl} 1 \mathrm{~S} 1=572.93$;

Negative ESI-MS, m/z: found for $\left(\left[\mathrm{B}_{10} \mathrm{Cl}_{9} \mathrm{~S}\left(n-\mathrm{C}_{4} \mathrm{H}_{9}\right)_{2}\right][\mathrm{HCOOH}]\right)^{-}=617.88$, calc. for $\mathrm{C} 9 \mathrm{H} 20 \mathrm{~B} 10 \mathrm{C} 19 \mathrm{O} 2 \mathrm{~S} 1=618.93$;

Negative ESI-MS, m/z: found for $\left(\left[\mathrm{B}_{10} \mathrm{Cl}_{9} \mathrm{~S}\left(n-\mathrm{C}_{4} \mathrm{H}_{9}\right)\right] \mathrm{H}\right)^{-}=516.86$, calc. for C4H10B10Cl9S1 = 516.86;

Negative ESI-MS, m/z: found for $\left(\left[\left(\mathrm{C}_{4} \mathrm{H}_{9}\right)_{4} \mathrm{~N}\right][\mathrm{HCOO}]_{2}\right)^{-}=332.28$, calc. for $\mathrm{C} 18 \mathrm{H} 38 \mathrm{~N} 1 \mathrm{O} 4=332.28$.

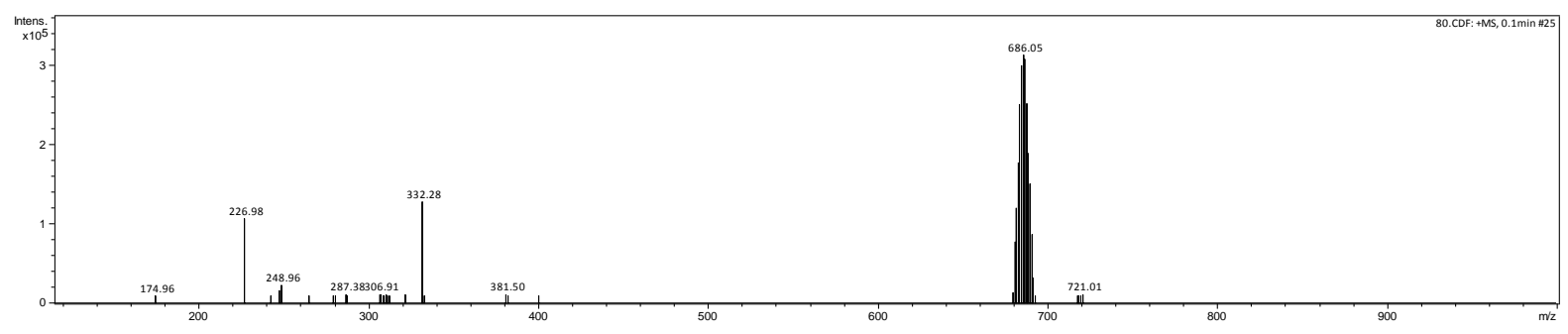




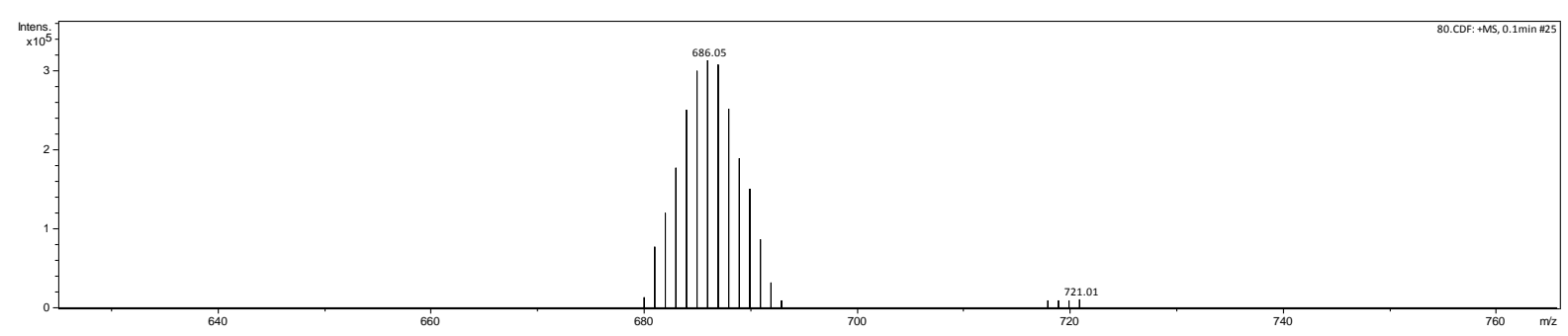

Fig. S57. ESI-MS spectrum of compound $\left.4\left(\left(\mathrm{C}_{4} \mathrm{H}_{9}\right)_{4} \mathrm{~N}\right)\left[\mathrm{B}_{10} \mathrm{Cl}{ }_{9} \mathrm{~S}\left(n-\mathrm{C}_{8} \mathrm{H}_{17}\right)_{2}\right]\right)$ in the negative ionization mode

Negative ESI-MS, m/z: found for $\left(\left[\mathrm{B}_{10} \mathrm{Cl}_{9} \mathrm{~S}\left(n-\mathrm{C}_{8} \mathrm{H}_{17}\right)_{2}\right]\right)^{-}=686.05$, calc. for $\mathrm{C} 16 \mathrm{H} 34 \mathrm{~B} 10 \mathrm{Cl} 19 \mathrm{~S} 1=658.05$;

Negative ESI-MS, m/z: found for $\left(\left[\left(\mathrm{C}_{4} \mathrm{H}_{9}\right)_{4} \mathrm{~N}\right][\mathrm{HCOO}]_{2}\right)^{-}=332.28$, calc. for $\mathrm{C} 18 \mathrm{H} 38 \mathrm{~N} 1 \mathrm{O} 4=332.28$.
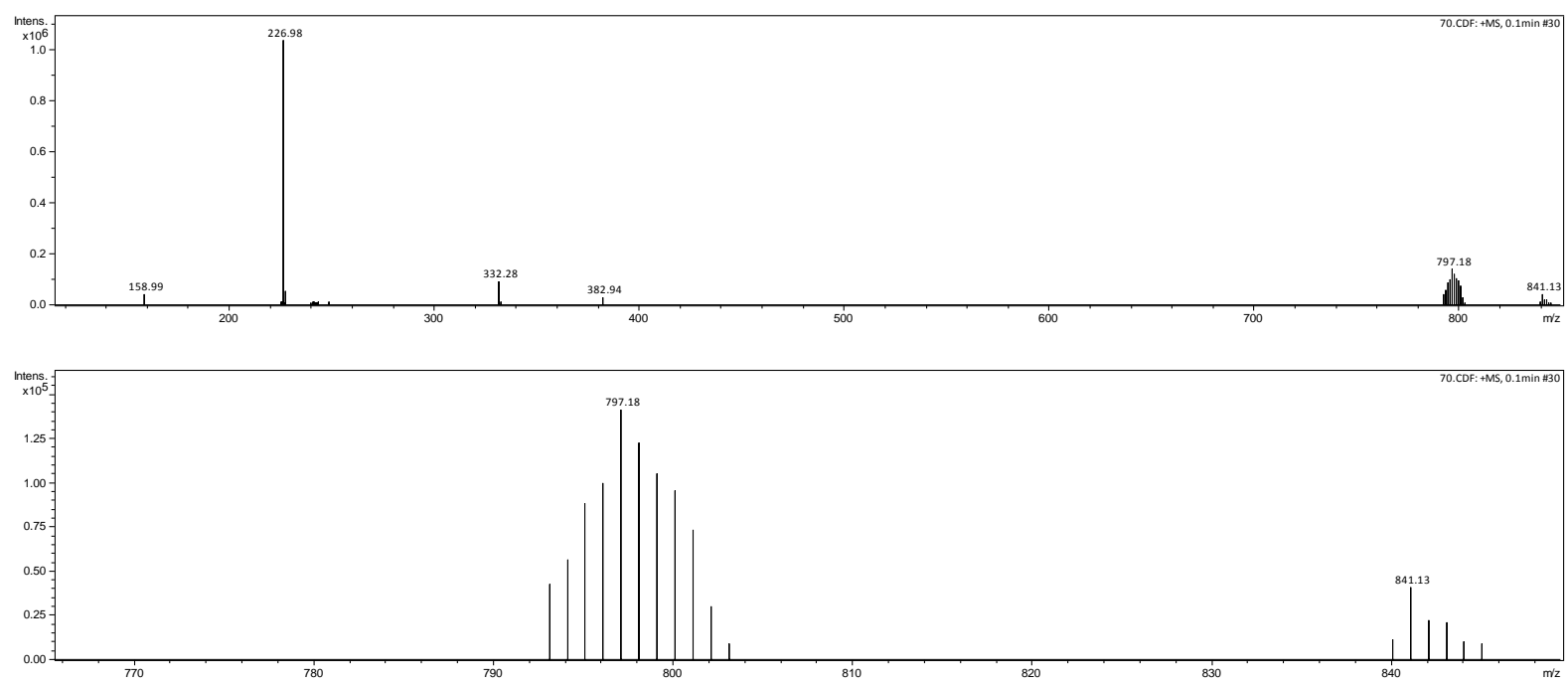

Fig. S58. ESI-MS spectrum of compound $\left.5\left(\left(\mathrm{C}_{4} \mathrm{H}_{9}\right)_{4} \mathrm{~N}\right)\left[\mathrm{B}_{10} \mathrm{Cl}{ }_{9} \mathrm{~S}\left(n-\mathrm{C}_{12} \mathrm{H}_{25}\right)_{2}\right]\right)$ in the negative ionization mode

Negative ESI-MS, m/z: found for $\left(\left[\mathrm{B}_{10} \mathrm{Cl}_{9} \mathrm{~S}\left(n-\mathrm{C}_{12} \mathrm{H}_{25}\right)_{2}\right]\right)^{-}=797.18$, calc. for C24H50B10C19S1 = 797.18;

Negative ESI-MS, m/z: found for $\left(\left[\mathrm{B}_{10} \mathrm{Cl}_{9} \mathrm{~S}\left(n-\mathrm{C}_{12} \mathrm{H}_{25}\right)_{2}\right][\mathrm{HCOOH}]\right)^{-}=841.13$, calc. for $\mathrm{C} 25 \mathrm{H} 52 \mathrm{~B} 10 \mathrm{Cl} 19 \mathrm{O} 2 \mathrm{~S} 1=843.18$;

Negative ESI-MS, m/z: found for $\left(\left[\left(\mathrm{C}_{4} \mathrm{H}_{9}\right)_{4} \mathrm{~N}\right][\mathrm{HCOO}]_{2}\right)^{-}=332.28$, calc. for C18H38N1O4 = 332.28.

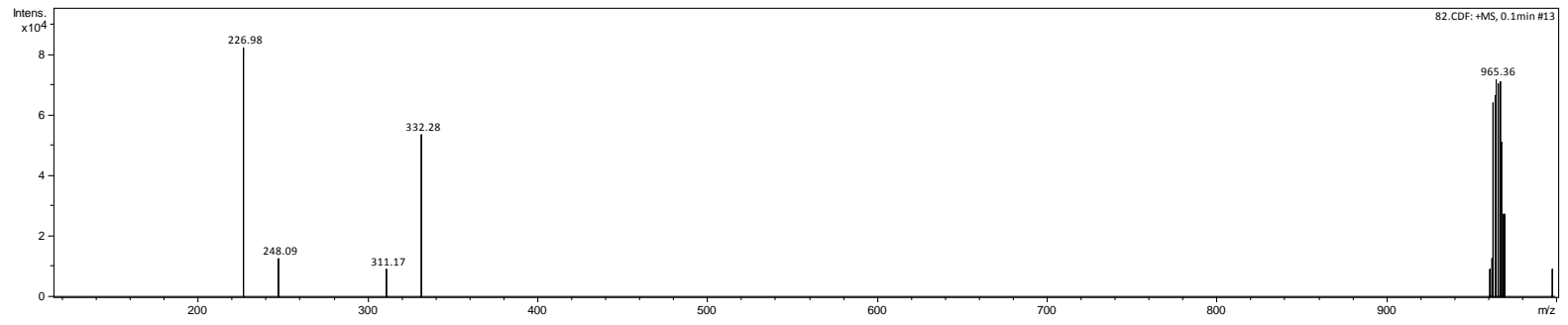




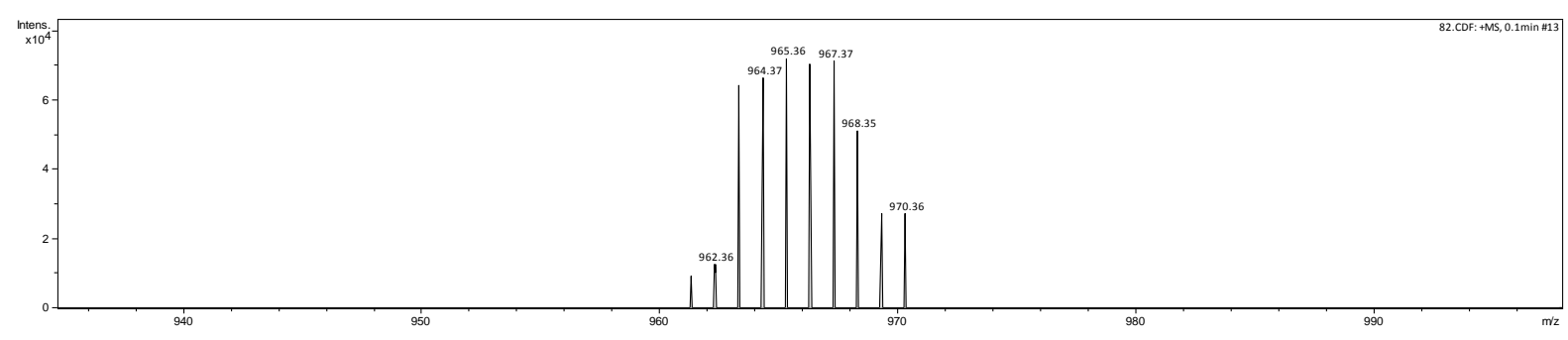

Fig. S59. ESI-MS spectrum of compound $\left.6\left(\left(\mathrm{C}_{4} \mathrm{H}_{9}\right)_{4} \mathrm{~N}\right)\left[\mathrm{B}_{10} \mathrm{Cl}_{9} \mathrm{~S}\left(n-\mathrm{C}_{18} \mathrm{H}_{37}\right)_{2}\right]\right)$ in the negative ionization mode

Negative ESI-MS, m/z: found for $\left(\left[\mathrm{B}_{10} \mathrm{Cl}_{9} \mathrm{~S}\left(n-\mathrm{C}_{18} \mathrm{H}_{37}\right)_{2}\right]\right)^{-}=965.36$, calc. for C36H74B10C19S1 = 966.36;

Negative ESI-MS, m/z: found for $\left(\left[\left(\mathrm{C}_{4} \mathrm{H}_{9}\right)_{4} \mathrm{~N}\right][\mathrm{HCOO}]_{2}\right)^{-}=332.28$, calc. for $\mathrm{C} 18 \mathrm{H} 38 \mathrm{~N} 1 \mathrm{O} 4=332.28$.
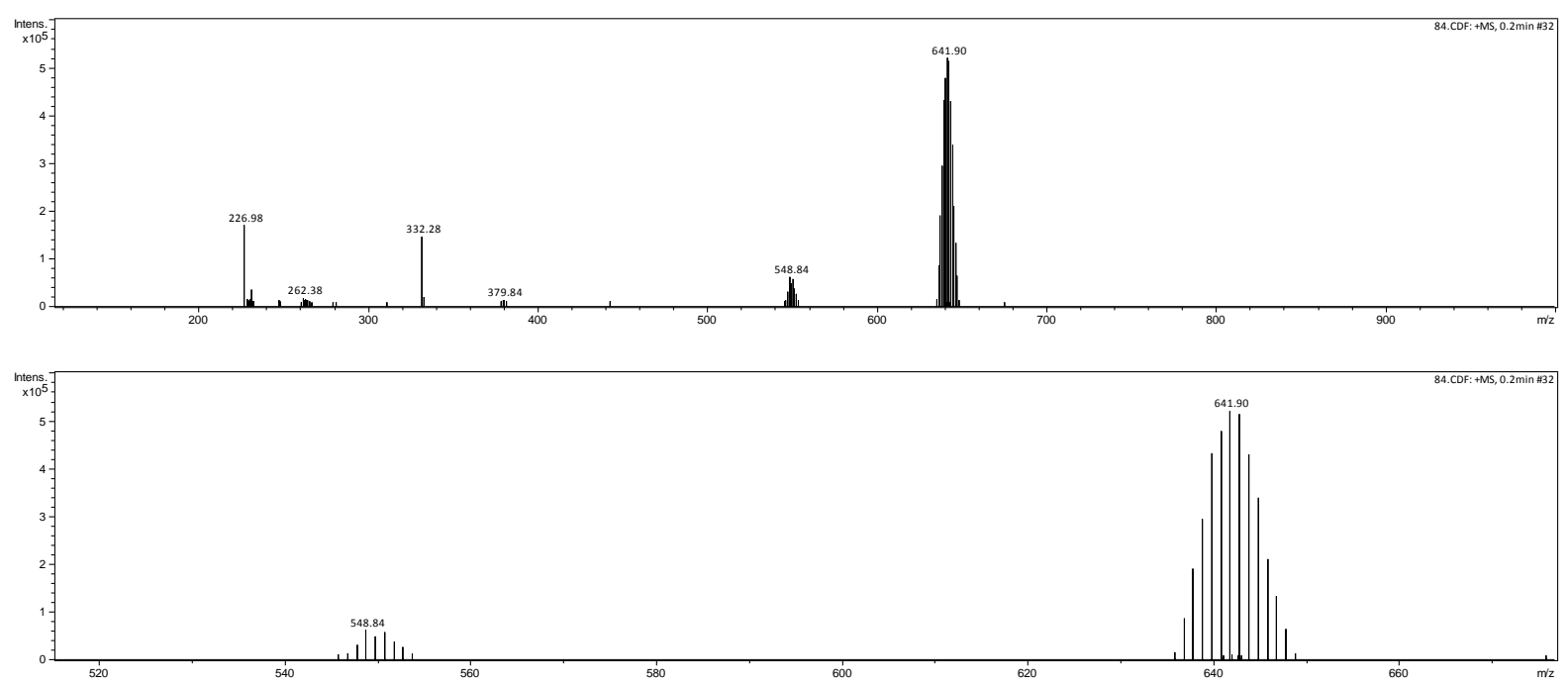

Fig. S60. ESI-MS spectrum of compound $\left.7\left(\left(\mathrm{C}_{4} \mathrm{H}_{9}\right)_{4} \mathrm{~N}\right)\left[\mathrm{B}_{10} \mathrm{Cl}_{9} \mathrm{~S}\left(\mathrm{CH}_{2} \mathrm{Ph}\right)_{2}\right]\right)$ in the negative ionization mode

Negative ESI-MS, m/z: found for $\left(\left[\mathrm{B}_{10} \mathrm{Cl}_{9} \mathrm{~S}\left(\mathrm{CH}_{2} \mathrm{Ph}\right)_{2}\right]\right)^{-}=641.90$, calc. for C14H14B10Cl9S1 = 640.89;

Negative ESI-MS, m/z: found for $\left(\left[\mathrm{B}_{10} \mathrm{Cl}_{9} \mathrm{~S}\left(\mathrm{CH}_{2} \mathrm{Ph}\right)\right] \mathrm{H}\right)^{-}=548.84$, calc. for C7H8B10C19S1 = 550.85;

Negative ESI-MS, m/z: found for $\left(\left[\left(\mathrm{C}_{4} \mathrm{H}_{9}\right)_{4} \mathrm{~N}\right][\mathrm{HCOO}]_{2}\right)^{-}=332.28$, calc. for $\mathrm{C} 18 \mathrm{H} 38 \mathrm{~N} 1 \mathrm{O} 4=332.28$.

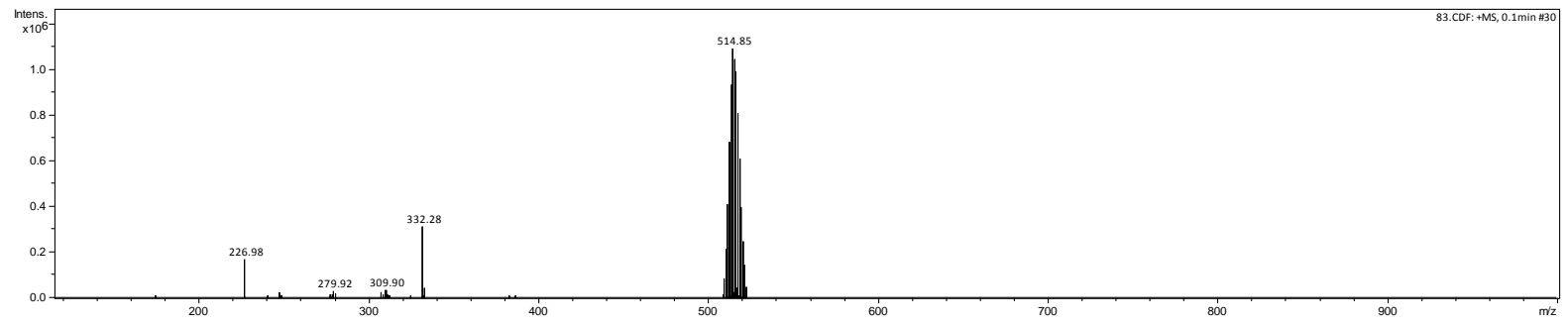




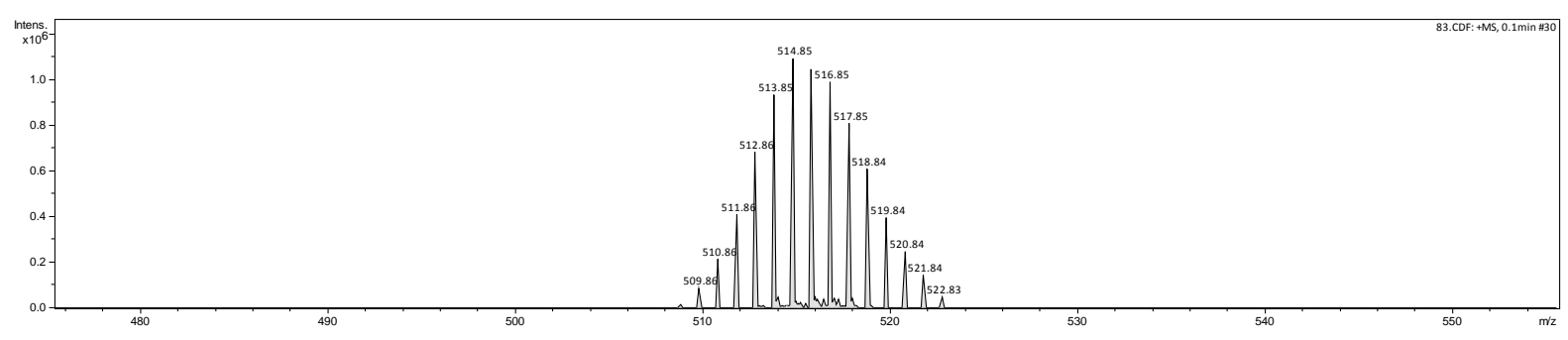

Fig. S61. ESI-MS spectrum of compound $8\left(\left(\mathrm{C}_{4} \mathrm{H}_{9}\right)_{4} \mathrm{~N}\right)\left[\mathrm{B}_{10} \mathrm{Cl}{ }_{9} \mathrm{~S}-\right.$ cyclo- $\left.\left.\mathrm{S}\left(\mathrm{CH}_{2}\right)_{4}\right]\right)$ in the negative ionization mode

Negative ESI-MS, m/z: found for $\left(\left[\mathrm{B}_{10} \mathrm{Cl}_{9}-\text { cyclo-S}\left(\mathrm{CH}_{2}\right)_{4}\right]\right)^{-}=514.85$, calc. for C4H8B10C19S1 = 514.85;

Negative ESI-MS, m/z: found for $\left(\left[\left(\mathrm{C}_{4} \mathrm{H}_{9}\right)_{4} \mathrm{~N}\right][\mathrm{HCOO}]_{2}\right)^{-}=332.28$, calc. for $\mathrm{C} 18 \mathrm{H} 38 \mathrm{~N} 1 \mathrm{O} 4=332.28$. 
Figure depicting the discussed noncovalent $\mathrm{B}-\mathrm{Cl}$...H interactions

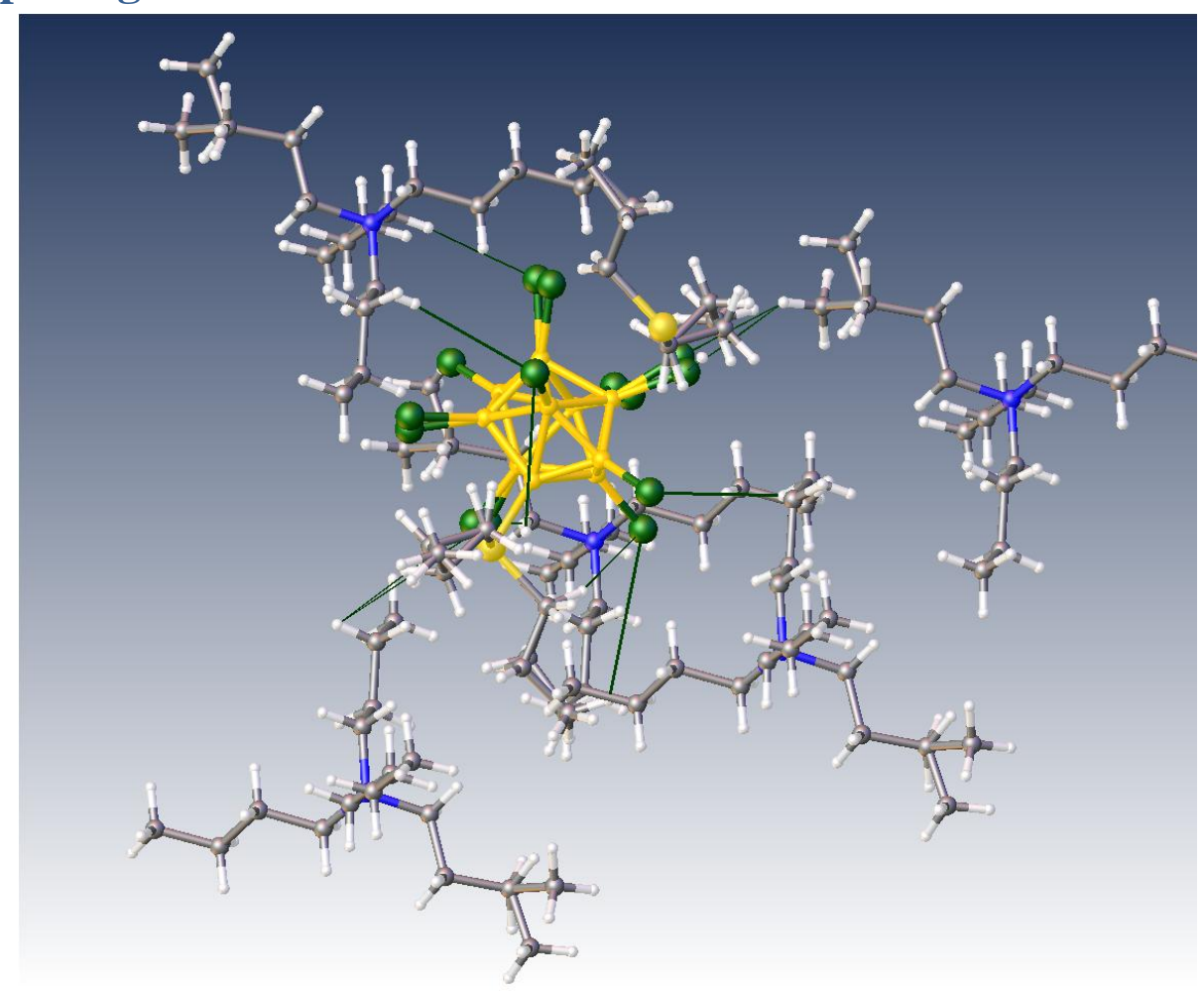

Fig. S62. Compound 2

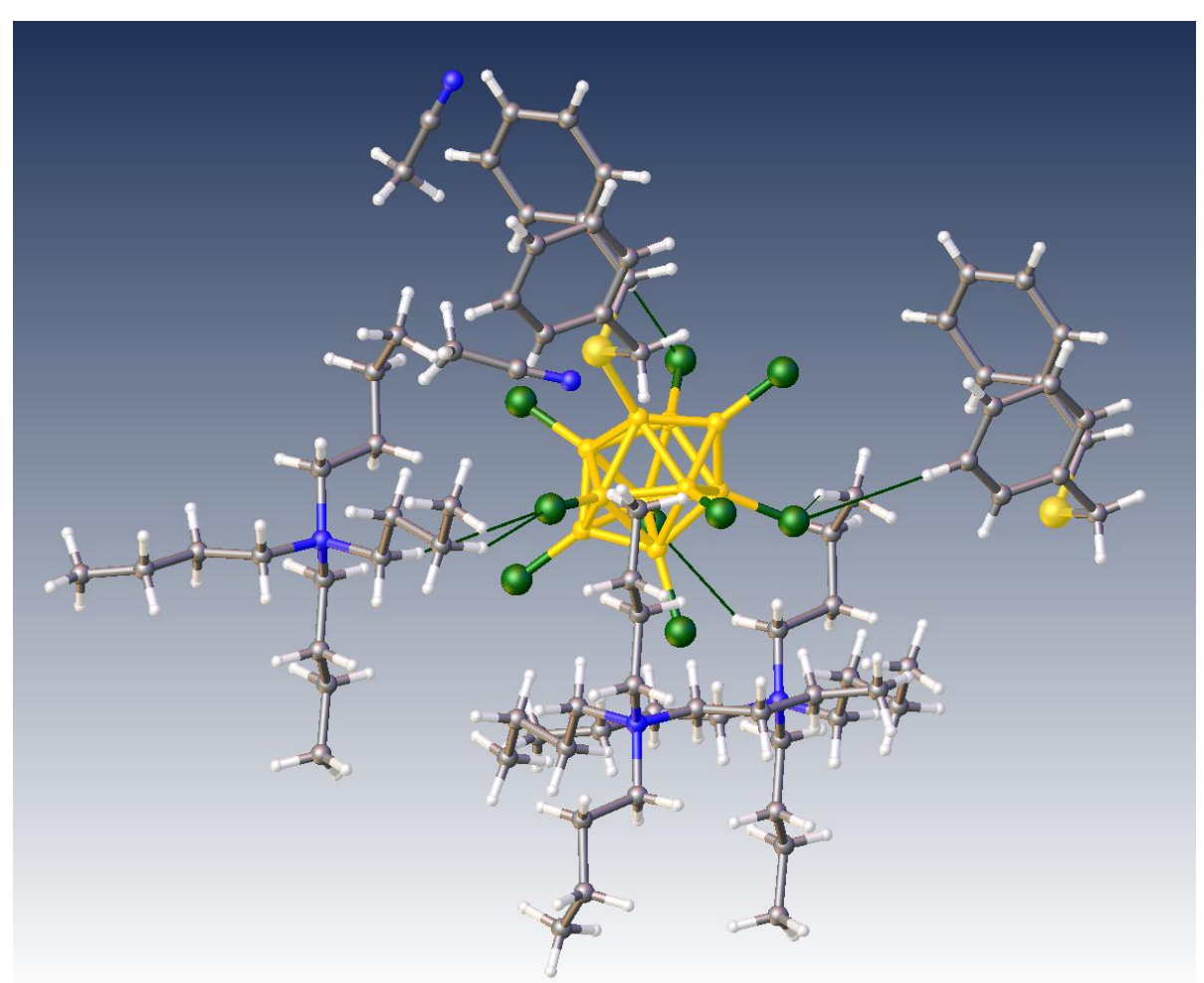

Fig. S63. Compound 7 


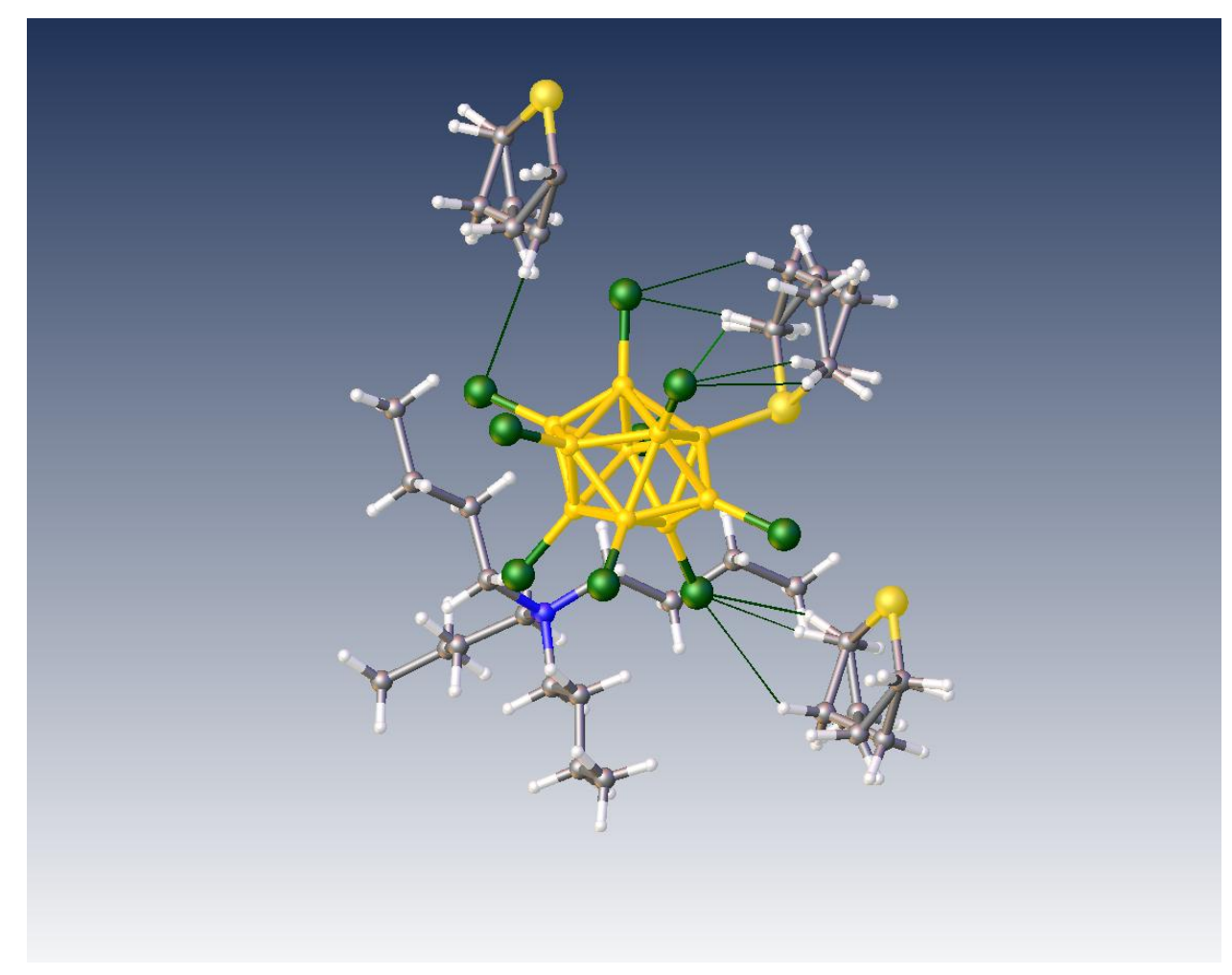

Fig. S64. Compound 8 